\section{To: (Receiving organization)} Distribution

5. Proj./Prog./Dept./Div.:

Tank 103, 219-S Facility at 222-S Lab/Waste Management/FP/HALO

8. Originator Remarks:

This document is being released into the supporting document system for retrievability purposes.

11. Receiver Remarks: 11A. Design Baseline Document? [] Yes [X] No For release.

\section{From: (originating organization)} 222-S Facility Planning

6. Design Authority/_Design Agent/Cog. Engr.:

R. K. Fuller
4. Related EDT NO.:
N/A

7. Purchase Order No.:

$N / A$

9. Equip./Component No.:

$\mathrm{N} / \mathrm{A}$

10. System/Bldg./Facility: 219-S at 222-S

12. Major Assm. Dwg. No.:

$N / A$

13. Permit/Permit Apptication No.: $\mathrm{N} / \mathrm{A}$

14. Required Response Date: $06 / 16 / 98$

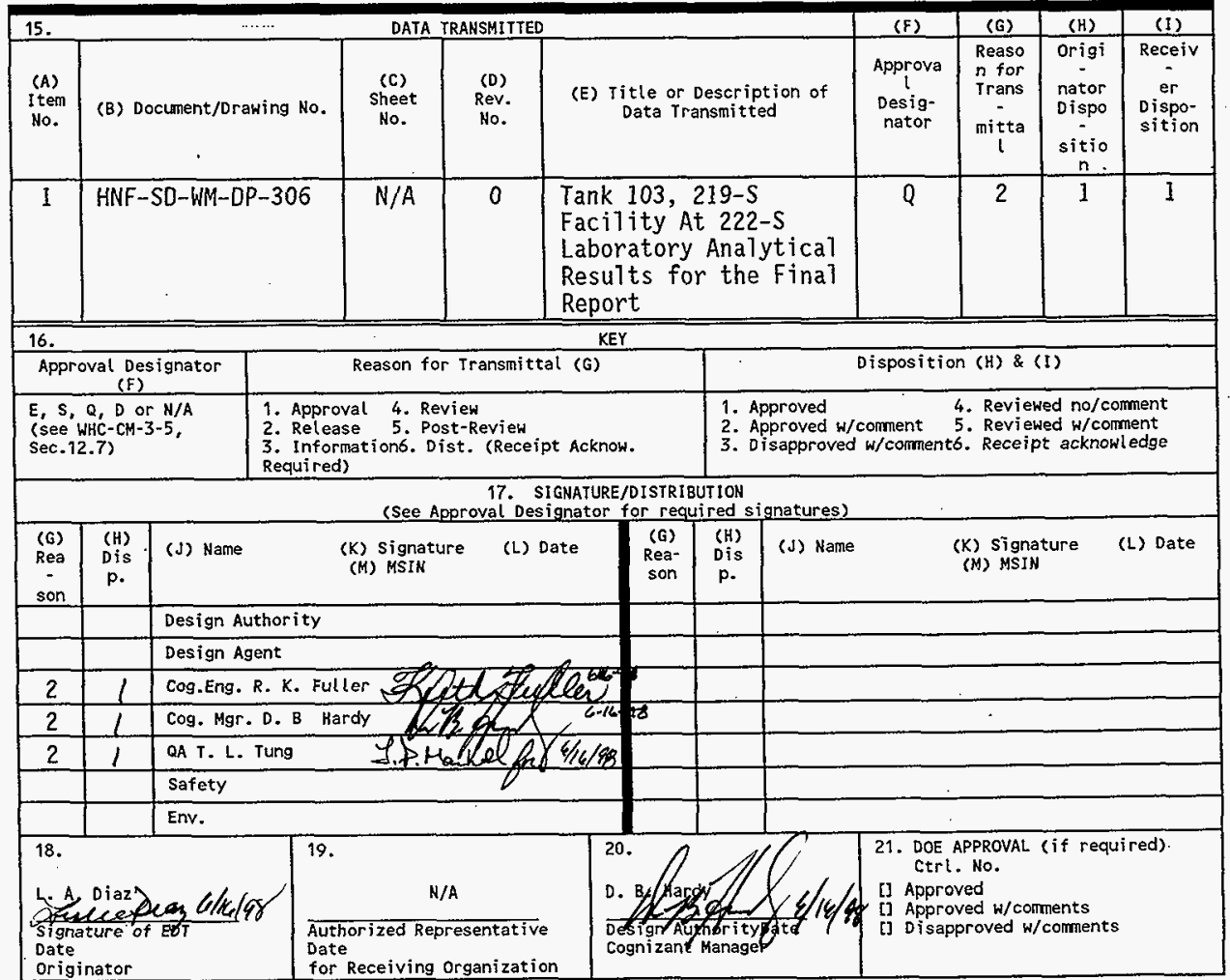




\title{
Tank 103, 219-S Facility at 222-S Laboratory Analytical Results for the Final Report
}

\author{
R. K. Fuiler
}

Waste Management Hanford, Inc., RichTand, WA 99352

U.S. Department of Energy Contract DE-AC06-96RL13200

$\begin{array}{lll}\text { EDT/ECN: } & \text { EDT-623158 } & \text { UC: } 2070 \\ \text { Org Code: } 7576 \mathrm{~B} & \text { Charge Code: MD378 } \\ \text { B\&R Code: } & \text { EW } 3120074 & \text { Total Pages: } 126\end{array}$

Key Words: Tank 103, 219-S, Lab Waste, AnaTytical Results, Final Report Abstract: N/A

TRADEMARK DISCLAIMER. Reference herein to any specific comercial product, process, or service by trade name, trademark, manufacturer, or otherwise, does not necessarily constitute or imply its endorsement, recommendation, or favoring by the United states Governnent or any agency thereof or its contractors or subcontractors.

Printed in the United States of America. To obtain copies of this document, contact: Document Control Services, P.0. Box 950, Mailstop H6-08, Richland WA 99352, Phone (509) 372-2420;

Fax (509) 376-4989.
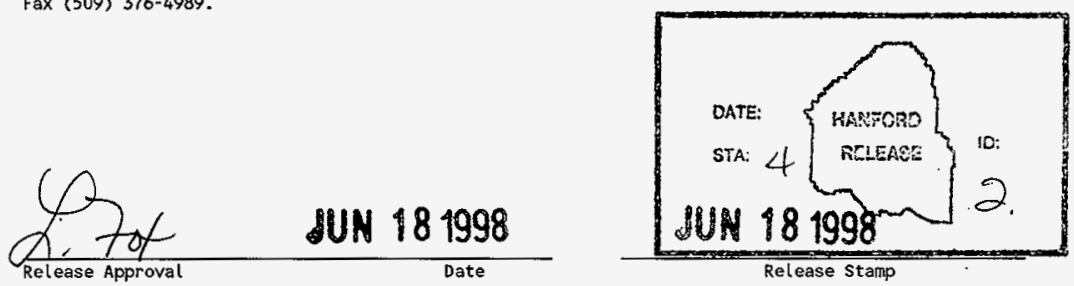

\section{Approved for Public Release}


HNF-SD-WM-DP-306, REV. 0

ANALYTICAL SERVICES

\title{
TANK 103, 219-S FACILITY AT 222-S LABORATORY ANALYTICAL RESULTS FOR THE FINAL REPORT
}

\author{
Project Coordinator: Keith Fuller \\ Prepared for the U.S. Department of Energy \\ Office of Environmental Restoration \\ and Waste Management \\ by \\ 222-S Laboratory \\ Waste Management of Hanford, Inc. \\ P. 0 . Box 700 \\ Richland, Washington
}


HNF-SD-HM-DP-306, REV. 0

THIS PAGE INTENTIONALLY LEFT BLANK 
HNF-SD-WM-DP-306, REV. 0

\section{TABLE OF CONTENTS}

Narrative ........................ I

PCB Analysis Report ..................... 4

Appendix A (LA-532-138) ................... 14

Appendix D (LA-523-136) . . . . . . . . . . . . . 17

CLP-Like Forms ..................... 20

CLP-Like MS/MSD Report ...................27

Extraction Benchsheet .................. 30

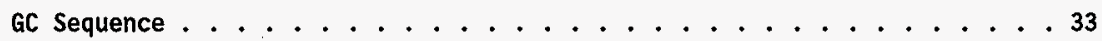

Initial Calibration Report . . . . . . . . . . . 39

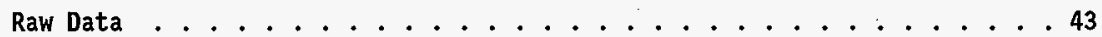

Continuing Calibration Verification ............ 106

Sample Breakdown ..................... . . 114

Requests for Sample Analysis ................ 117

This Document consists of pages 1 through 121 . Pages $i$ i, 2, 5, 15, 18, 21, 28, $34,40,44,107,115$, and 118 were initially left blank.

This document also includes page 3.1 . 
HNF-SD-WM-DP-306, REV. 0

NARRAT IVE 
HNF-SD-WM-DP-306, REV. 0

THIS PAGE INTENTIONALLY LEFT BLANK 


\section{HNF-SD-WM-DP-306 REV. 0}

\section{2-S HANFORD ANALYTICAL LABORATORY OPERATIONS \\ TANK 103, 219-S FACULITY AT 222-S LABORATORY ANALYTICAL RESULTS FOR THE FINAL REPORT}

This is the final report for the polychlorinated biphenyls analysis of Tank-103 (TK-103) in the 219-S Facility at 222-S Laboratory.

Twenty 1-liter bottles (Sample numbers S98S000074 through S98S000093) were received from TK-103 during two sampling events, on May 5 and May 7, 1998. The samples were centrifuged to separate the solids and liquids. The centrifuged sludge was analyzed for PCBs as Aroclor mixtures. The results are discussed on page 6 .

The sample breakdown diagram (Page 114) provides a cross-reference of sample identification of the bulk samples to the laboratory identification number for the solids. The request for sample analysis (RSA) form is provided as Page 117. The raw data is presented on Page 43.

\section{Sample Description, Handling, and Preparation}

Twenty samples were received in the laboratory in 1-Liter bottles. The first 8 samples were received on May 5, 1998. There were insufficient solids to perform the requested PCB analysis and 12 additional samples were collected and received on May 7, 1998. Breakdown and subsampling was performed on May 8, 1998. Sample number S98S000084 was lost due to a broken bottle.

Nineteen samples were centrifuged and the solids were collected in 8 centrifuge cones. After the last sample was processed, the solids were consolidated into 2 centrifuge cones. The first cone contained 9.7 grams of solid and 13.0 grams was collected in the second cone. The wet sludge from the first centrifuge cone was submitted to the laboratory for PCB analysis (sample number S98S000102). The other sample portion (S98S000103) was retained for possible additional analyses.

\section{Analytical Results}

\section{Polychlorinated Biphenyl (PCB) Analysis}

Analysis for polychlorinated biphenyl (PCB) compounds was performed on the wet centrifuged solids. Aroclors were observed in the sample and reported as Aroclor 1254 with 


\section{HNF-SD-WM-DP-306 REV. 0}

an average result of approximately $46 \mathrm{ppm}$. A dètailed discussion of the results is presented on Page 6. 
HNF-SD-WM-DP-306, REV, 0

PCB ANALYSIS REPORT

4 
HNF-SD-WM-DP-306, REV. 0

THIS PAGE INTENTIONALLY LEFT BLANK 
HNF-SD-WM-DP-306 REV. 0

POLYCHLORINATED BIPHENYLS (PCB) ANALYSIS REPORT

FOR SOLID SAMPLE FROM 219 S TANK 103

$$
\begin{gathered}
\text { 222-S Laboratory } \\
\text { May } 1998 \\
\text { submitted by }
\end{gathered}
$$

GA RosS

Waste Management Federa? Services of Hanford 


\section{HNF-SD-WM-DP-306 REV. 0}

\section{POLYCHLORINATED BIPHENYLS (PCB) ANALYSIS REPORT FOR SOLID SAMPLE FROM 219 S TANK 103}

\section{SAMPLE ANALYSIS REPORTED}

One waste sample with centrifuged solids was obtained from tank 103 of 2195 via a peristaltic pump equipped with a stainless steel tube and Norprene tubing (Phthalate free). The sample, obtained in a glass jar with Teflon ${ }^{2}$ lid, was analyzed (with duplicate, matrix spike, matrix spike duplicate, method blank and 1 aboratory control sample) for PCBs as Aroclor mixtures by the Direct Analysis Team. A soxhlet extraction procedure was used to extract the Aroclors from the sample. Analysis was performed using dual column confirmation gas chromatography/ electron capture detection (GC/ECD). Extraction closely follows SW-846 method 3540C, and the analysis follows SW846 method 8082 .

SAMPLE DESCRIPTION, HANDLING, AND PREPARATION

The 1aboratory sample was received as follows: S985000102 Centrifuged solids from 19 1-1iter jars (S98S000074-93, except S98S000084, which broke) of TK-103 1iquid.

The sample preparations and analyses reported were on a as received, post centrifugation, basis. The sample was green in color and had the consistency of putty. After one minute of stirring, four one gram aliquots were taken from the parent sample jar for analysis. Due to limited sample and radiation dose concerns a nominal one gram of sample was used for each analysis. Extraction of the samples was started on May 11, 1998 in a radiological fume hood.

Preparation Procedure: LA-523-138 Rev. A2, Soxhlet Extraction of Solid Samples for Semivolatile and/or Pesticide and/or PCB Analysis, Waste Management Federal Services of Hanford, Inc. for Fluor Daniel Hanford, Inc., Richland, Washington.

Preparation Location: Room 4P, 222-S Laboratory, 200W Area, Hanford Site

Preparation Type:

Soxhlet Extraction using methylene chloride, KudernaDanish concentration with solvent exchange to hexane and cleanup by sulfuric acid.

Sample Extract Storage: 4 degrees Celsius in darkness

1 Norprene is a trademark of Norton Corporation

2 Tefion is a trademark of E.I. duPont de Nemours \& Company, Incorporated.

3 Aroclor is a trademark of the Monsanto Company 
ANALYSIS METHOD

\section{HNF-SD-WM-DP-306 REV. 0}

GC/ECD Procedure: $\quad$ LA-523-136 Rev. A1, Gas Chromatograph Analysis of Pesticides and PCBS - SW-846, Waste Management Federal Services of Hanford, Inc. for Fluor Daniel Hanford, Inc., Richland, Washington.

GC/ECD instrumentation: HP-5890 Series II gas chromatograph with dual on-column injection, XTI-5 and DB-608 columns, and dual electron capture (EC) Detectors, (property number WC 44940).

Location: $\quad$ Room 4TUV, 222-S Laboratory

The analyses were performed on May 21, 1998 on the extracts obtained on May 13, 1998. Due to the high concentrations of PCBs present, the extracts were analyzed on May 21, 1998 with a dilution was necessary in order to quantify the Aroclors present. The (100X) dilution of the sample and sample duplicate was accomplished by diluting $10.0 \mu \mathrm{L}$ of the extract with $990 \mu \mathrm{L}$ of hexane. The laboratory control sample, matrix spike and matrix spike duplicate were accomplished by adding $1250 \mu \mathrm{L}$ of the $40 \mu \mathrm{g} / \mathrm{mL}$ Aroclor 1254 spike, and $400 \mu \mathrm{L}$ of $40 \mu \mathrm{g} / \mathrm{mL}$ surrogate mix.

QUALITY CONTROL

Due to limited sample amount and radiological issues the extraction portion of the procedure was scaled down from SW-846 method $3540 \mathrm{C}$. Appendix $A$ of LA-523-138 details the deviations to method $3540 \mathrm{C}$ that are contained in the procedure. The analys is follows SW-846 method 8082 . Deviations from method 8082 are detailed in appendix $D$ of $L A-523-136$. Nominally, one gram of sample was extracted per analytical result.

Five point calibrations were run for the following Aroclors: 1016, 1254, 1260, and 1248. Concentration ranges went from $20 \mathrm{ng} / \mathrm{mL}$ to $400 \mathrm{ng} / \mathrm{mL}$. Three point calibrations were performed for Aroclors 1221, 1232, and 1242. The sample analysis was bracketed with a second mid-level standard Aroclor 1254. An instrument blank, method blank and LCS were analyzed during the sequence of sample analysis. Calculations for total area of Arocior 1254 calibration were performed to assess the linearity, CCV, and sample quantitations (page 3 P2).

Quantitation was performed using LA-523-136 option 2 (total area) for Aroclor 1254. A primary column and the secondary column was used to confirm identification. A detalled description of the specific procedural requirements is available in the analytical procedure LA-523-136. Laboratory bench sheets, data, chromatograms, and reports are maintained in room 25, 2704-S at the 222$S$ Laboratory. The initial calibration information and data is located on the HP UNIX computer system, Chemsys I. 


\section{HNF-SD-WM-DP-306 REV. 0}

Table I summarizes the $\mathrm{QC}$ requirements and adherence.

Table I.

\begin{tabular}{|c|c|}
\hline QC Parameter & Comments \\
\hline Target Compounds & $\begin{array}{l}\text { Detection levels are based on sample size and the } \\
\text { determination of Method Detection Limits (MDLs) from } \\
\text { multiple injections of a low standard. Requirements } \\
\text { were met. A UX flag denotes a compound was manually } \\
\text { deleted in review of the data. }\end{array}$ \\
\hline Surrogate Recoveries & $\begin{array}{l}\text { Surrogate spiking was performed on all samples using } \\
\text { Tetrachloro-m-xylene (TCX) and Decachlorobiphenyl } \\
\text { (DCB). A summary of the surrogate recoveries is } \\
\text { provided in Table II. All surrogate recoveries were } \\
\text { within acceptable administrative limits. }\end{array}$ \\
\hline $\begin{array}{l}\text { Matrix Spike } \\
\text { Recovery }\end{array}$ & $\begin{array}{l}\text { A-matrix spike (MS) and matrix spike duplicate (MSD) } \\
\text { using Aroclor } 1254 \text { was performed. Recoveries were } \\
\text { acceptable (Table III). }\end{array}$ \\
\hline Method Blank Summary & A method blank was extracted and met requirements. \\
\hline $\begin{array}{l}\text { Initial Method } \\
\text { Detection Limit } \\
\text { Determination }\end{array}$ & $\begin{array}{l}\text { Multiple extraction and analysis of a low } \\
\text { concentration standard. Requirements were met. } \\
\text { (For } 2 \mathrm{~g} \text { sample the MDL for Aroclor } 1248=3.71 \\
\mathrm{ug} / \mathrm{kg} \text {, documented in files). }\end{array}$ \\
\hline Initial Calibration & $\begin{array}{l}\text { Five point calibrations were performed for Aroclors } \\
1016,1248,1254 \text {, and. } 1260 \text {. Concentrations ranged } \\
\text { from } 20 \mathrm{ng} / \mathrm{mL} \text { to } 400 \mathrm{ng} / \mathrm{mL} \text {. Three point calibrations } \\
\text { were performed for Aroclors } 1221 \text {, } 1232 \text {, and } 1242 \text { at } \\
100 \text { or } 200 \mathrm{ng} / \mathrm{mL} \text { to } 400 \text { or } 800 \mathrm{ng} / \mathrm{ml} \text {. By total area, } \\
\text { the } \mathrm{RSO} \% \text { for Aroclor } 1254 \text { was } 6.89 \% \text {. }\end{array}$ \\
\hline $\begin{array}{l}\text { Carry-over } \\
\text { evaluation }\end{array}$ & $\begin{array}{l}\text { An instrument blank was run after the calibration } \\
\text { standards. No PCB carry-over was detected, but a } \\
\text { large peak at } 20.27 \text { minutes, and four other peaks } \\
\text { were previously observed in the trip bottle blank or } \\
\text { sample matrix (on } 51398 . b \text { ) The non-PCB peak areas at } \\
12.97 \text {, } 14.77 \text {, 15.16, and } 17.34 \text { are subtracted from } \\
\text { the sample total area if they were present in the } \\
\text { chromatogram. }\end{array}$ \\
\hline $\begin{array}{l}\text { Continuing } \\
\text { Calibration } \\
\text { Verifications (CCV) }\end{array}$ & $\begin{array}{l}\text { Initial calibration was performed during the sample } \\
\text { sequence. Aroclor } 1254 \text { was used to assess } \\
\text { instrument performance before and after the samples } \\
\text { were analyzed on it. The value found for the } .200 \mathrm{ng} \\
\text { Aroclor } 1254 \text { standard after the samples was } .214 \mathrm{ng} \text {. } \\
\text { This is } 107 \% \text { of the amount, and is within the } 15 \% \\
\text { acceptable. }\end{array}$ \\
\hline $\begin{array}{l}\text { Laboratory Control } \\
\text { Sample }\end{array}$ & $\begin{array}{l}\text { A matrix spike blank was used as a Laboratory } \\
\text { Control Sample to assess method performance. This } \\
\text { contained Aroclor } 1254 \text {. Recovery was } 85 \% \text {, and met } \\
\text { requirements of } 70-130 \% \text {. }\end{array}$ \\
\hline
\end{tabular}




\section{HNF-SD-WR-DP-306 REV. 0}

\section{RESULTS / DISCUSSION}

The Aroclor 1254 was observed to be predominant in the sample at high concentrations. A dilution was necessary to bring the amounts on column within the calibration curve. After careful comparison, it was noted that 1248 may be present, but the predominant pattern is 1254 . Therefore the total area method was used to quantify the Arocior as 1254. This may cause a high bias compared to option 1 quantitation for 1254 because some minor non-PCB peaks are included as Aroclor due to the complexity of the chromatograms. The $400 \mathrm{ng} / \mathrm{mL}$ calibration file was used for the total area quantitation of Aroclors in the samples as Aroclor 1254. The total number of PCBs quantified (per option 2, LA-523-136) in sample S98S00102, as received, post centrifugation, as Aroclor 1254 is $47.7 \mathrm{mg} / \mathrm{Kg}$ and $44.8 \mathrm{mg} / \mathrm{Kg}$ for the sample and duplicate, respectively. The average total PCBs as Aroclors value is $46 \mathrm{mg} / \mathrm{Kg}$, with an RPD of $6.2 \%$.

The Laboratory Control Spike (LCS) recovery was good. The Aroclor mixture of 1254 was used as an LCS, and was recovered at $85 \%$.

Surrogate spiking was performed on all samples using Tetrachloro-m-xylene (TCX) and DecachiorobiphenyT (DCB). and is summarized in Table II for the instrument blanks, method blank, LCS, and undiluted sample, duplicate, No control limits have been established for these compounds in this matrix yet. According to U.S. Environmental Protection Agency advisory 1 imits, set at 50$150 \%$, surrogate recoveries for these samples are acceptable. Surrogate concentrations were determined by the 5-point calibration

Table II.

SURROGATE RECOVERIES (\%)

\begin{tabular}{|c|c|c|}
\hline Sample & TCX (\%) & DCB (\%) \\
\hline-- & - & -- \\
\hline Method Blank & 84 & 89 \\
\hline LCS & 79 & 78 \\
\hline S98S000102/100 & 87 & 96 \\
\hline S98S000102DUP/100 & 80 & 82 \\
\hline S98S000102MS/200 & 94 & 108 \\
\hline S98S000102MSD/200 & 93 & 96 \\
\hline-- & -- & -- \\
\hline
\end{tabular}

All surrogates within advisory limits of $50-150 \%$. 
HNF-SD-WM-DP-306 REV. 0

Table III,

SPIKE RECOVERIES (\%)

\begin{tabular}{|c|c|}
\hline Sample & Aroclor $1254(\%)$ \\
\hline Spike & 75.49 \\
\hline Spike Duplicate & 80.05 \\
\hline \hline 1254 Spike RPD & 5.86 \\
\hline
\end{tabular}

Table IV. AROCLOR RESULTS

\begin{tabular}{|c|c|c|}
\hline AROCLOR & S98S000102 (ug/g) & S98S000102DUP (ug/g) \\
\hline $\begin{array}{c}\text { TOTAL } \\
\text { (per Option 2 as 1254) }\end{array}$ & $(47.66)$ & $(44.79)$ \\
\hline Average & 46 & $6.2 \mathrm{RPD}$ \\
\hline
\end{tabular}




\section{HNF-SD-WMA-DP-306 REV. 0}

Calculation of PCBs per option 2 (total PCB area method)

Reason: Aroclor mixture of 1248 and 1254 observed in sample. Quantitation by 3-peak method, option 1 results in identification and quantification of one of the peaks as both aroclors. This creates an error. Option 2 permits quantitation of the total aroclors assuming the response most closely matches that of the more prevalent Aroclor.

According to the formula:

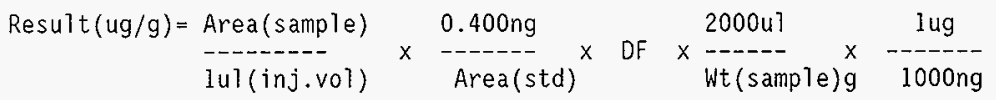

The results were as follows (4 digits used to allow RPD calculations):

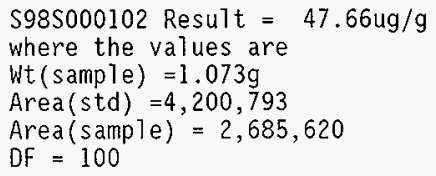

S98S000102Dup Resu1t $=44.79 u g / g$

where the values are

Wt $($ sample $)=1.117 \mathrm{~g}$

Area (std) $=4,200,793$

Area (sample) $=2,626,878$

$D F=100$

S98S000102MS Result $=81.82 \mathrm{ug} / \mathrm{g}$

where the values are

Wt $($ sample $)=1.105 \mathrm{~g}$

Area $($ std $)=4,200,793$

Area (spiked sample) $=2,373,646$

$D F=200$

S98S000102MSD Result $=84.72 \mathrm{ug} / \mathrm{g}$

where the values are

Wt $($ sample $)=1.080 \mathrm{~g}$

Area $($ std) $=4,200,793$

Area (spiked sample) $=2,402,355$

$D F=200$ 


\section{HNF-SD-WM-DP-306 REV. 0}

File:C: \wpdata \ross\PCBtk103.gar ANALYST SPODOCDTE 5-29-98REVIEWER DATE 
HNF-SD-HM-DP-306, REV. 0

APPENDIX A

(LA-532-138)

14 
HNF-SD-HM-DP-306, REV. 0 $\because$

THIS PAGE INTENTIONALLY LEFT BLANK

15 
Appendix A

Comparison to SW-846

Method 3540C, Soxhlet Extraction, Revision 3, December 1996

\begin{tabular}{|c|c|c|c|}
\hline SW-846 States: & Procedure States: & Type of Change, & $\begin{array}{l}\text { Reason for } \\
\text { Change? }\end{array}$ \\
\hline $\begin{array}{l}\text { Section } 7.2 \text { : Use } \\
5 \text { to } 10 \text { grams of } \\
\text { sample }\end{array}$ & $\begin{array}{l}\text { Step 9.5: Use } 1 \\
\text { to } 10 \text { grams of } \\
\text { sample }\end{array}$ & $\begin{array}{l}\text { Deviation- allows } \\
\text { scale down of the } \\
\text { sample and spike } \\
\text { additions } \\
\text { appropriately } \\
\text { (cut by } \\
\text { approximately } \\
1 / 4 \text { ) }\end{array}$ & $\begin{array}{l}\text { Due to limited } \\
\text { amounts of } \\
\text { samples, waste } \\
\text { minimization and } \\
\text { ALARA concerns, } \\
\text { smaller amounts } \\
\text { of sample may } \\
\text { need to be used. }\end{array}$ \\
\hline $\begin{array}{l}\text { Section } 7.4: \text { Use } \\
300 \mathrm{~mL} \text { solvent }\end{array}$ & $\begin{array}{l}\text { Step 9.5: Use } 90 \\
\mathrm{~mL} \text { solvent. }\end{array}$ & $\begin{array}{l}\text { Deviation- scale } \\
\text { down of the } \\
\text { sample, solvent, } \\
\text { and spike } \\
\text { additions } \\
\text { appropriately } \\
\text { (cut by } \\
\text { approximately } \\
1 / 4 \text { ) }\end{array}$ & $\begin{array}{l}\text { See above. This } \\
\text { also reduces the } \\
\text { amount of methyl- } \\
\text { ene chloride } \\
\text { evaporated up the } \\
\text { hood. }\end{array}$ \\
\hline $\begin{array}{l}\text { Section 7.4: Use } \\
500 \mathrm{~mL} \text { round } \\
\text { flask. }\end{array}$ & $\begin{array}{l}\text { Step 9.11: Use } \\
125 \mathrm{~mL} \text { round } \\
\text { flask with flat } \\
\text { bottom. }\end{array}$ & Deviation & $\begin{array}{l}\text { Smaller flasks } \\
\text { provide efficient } \\
\text { extraction in } \\
\text { limited hood } \\
\text { space. }\end{array}$ \\
\hline $\begin{array}{l}\text { Section } 7.10 \text { : } \\
\text { Add approximately } \\
50 \mathrm{~mL} \text { exchange } \\
\text { solvent once. }\end{array}$ & $\begin{array}{l}\text { Steps } 9.16 .1- \\
9.16 .2: \text { Add } 30 \\
\mathrm{~mL} \text { of hexane } \\
\text { three times }\end{array}$ & Deviation & $\begin{array}{l}\text { Repeating process } \\
\text { efficiently } \\
\text { removes first } \\
\text { solvent residue. }\end{array}$ \\
\hline $\begin{array}{l}\text { Section } 7.12 .2 \text { : } \\
\text { Place the } \\
\text { concentrator tube } \\
\text { in a warm bath } \\
\left(35^{\circ} \mathrm{C}\right) \text { and } \\
\text { evaporate to } 0.5 \\
\mathrm{~mL} . .\end{array}$ & $\begin{array}{l}9.25 .1: \text { Place } \\
\text { the tube in the } \\
\text { Reacti-vap } \\
\text { evaporator } \\
\text { assembly, with } \\
\text { the heating } \\
\text { module preheated } \\
\text { to } 40^{\circ} \mathrm{C} \text {... } \\
\text { semivolatiles are } \\
\text { collected when } \\
\text { the MeCl } 7 \text { level } \\
\text { reaches } 2 \mathrm{~mL} \text {. }\end{array}$ & Substitution & $\begin{array}{l}\text { Heat transfer is } \\
\text { done with a heat- } \\
\text { ing block instead } \\
\text { of a bath, } \\
\text { temperature } \\
\text { differences are } \\
\text { nomina?. A final } \\
\text { volume of } 2 \mathrm{~mL} \\
\text { was necessary to } \\
\text { prevent losses. }\end{array}$ \\
\hline
\end{tabular}

Procedure No.

LA-523-138

Rev Mod

Page

A-2

19 of 23 
HNF-SD-WM-DP-306, REV. 0

APPENDIX B

(LA-532-136) 
HNF-SD-WM-DP-306, REV. 0

$\because$

THIS PAGE INTENTIONALLY LEFT BLANK 


\section{Appendix D \\ Comparison to $\mathrm{SW}-846^{8}$}

Method 8082, Revision 0, December 1996, Polychlorinated Bipheny7s (PCBs) By Gas Chromatography

\begin{tabular}{|c|c|c|c|}
\hline SW-846 states: & Procedure States: & Type of Change: & Reason for Change: \\
\hline $\begin{array}{l}\text { Table } 3 \text { or Step 2.5: (GC } \\
\text { Operating Conditions or } \\
\text { summary) Injection } \\
\text { volume: } 2 \mu \mathrm{L} \text { (with a } \\
0.54 \mathrm{~mm} \text { id. megabore } \\
\text { column). or Step } 7.4 .5 \\
\text { A } 2-\mu \mathrm{L} \text { injection of each } \\
\text { cal ibration standard is } \\
\text { recommended. other } \\
\text { injection volumes may be } \\
\text { used if the analyst can } \\
\text { demonstrate adequate } \\
\text { sensitivity for the } \\
\text { compounds. }\end{array}$ & $\begin{array}{l}\text { Step 10.1: Gas } \\
\text { Chromatograph Operating } \\
\text { Conditions - Injection } \\
\text { Volume: } 1 \mu \mathrm{L}\end{array}$ & Substitution & $\begin{array}{l}\text { Smaller injection } \\
\text { needed for mid-bore } \\
\text { column ( } 0.32 \mathrm{~mm} \text { id). } \\
\text { otherwise injection of } \\
\text { oil sample extracts } \\
\text { will compromise the } \\
\text { system. The "Suggested" } \\
\text { calibration levels of } \\
100,150,200,400 \text {, and } \\
600 \mathrm{ng} / \mathrm{mL} \text { extends above } \\
\text { the linear cal ibration } \\
\text { range for a } 1 \mu \mathrm{L} \\
\text { injection. Hence the } \\
\text { calibration levels } \\
\text { selected are at } 20,50 \text {, } \\
100,200, \text { and } 400 \\
\text { ng/mL. }\end{array}$ \\
\hline $\begin{array}{l}\text { Step 5.5: Stock } \\
\text { standard solutions }(1000 \\
\mathrm{mg} / \mathrm{L})\end{array}$ & $\begin{array}{l}\text { Step } 4.1 .2: \text {...the oc } \\
\text { reference sample } \\
\text { concentrate should } \\
\text { contain the most repre- } \\
\text { sentative multicomponent } \\
\text { parameter at a concen- } \\
\text { tration of } 50 \mathrm{mg} / \mathrm{L} \text {. (In } \\
\text { fact, } 40 \mathrm{mg} / \mathrm{L} \text { Standards } \\
\text { custom made by Restek } \\
\text { are used so that the } \\
\text { threshold limit of } 50 \\
\text { PPM will not be met or } \\
\text { exceeded.) }\end{array}$ & $\begin{array}{l}\text { Deviation- scale down of } \\
\text { the spike additions } \\
\text { appropriately (cut by } \\
\text { approximately } 1 / 100) \text {. }\end{array}$ & $\begin{array}{l}\text { ISCA Regulation } \\
\text { concerns, waste } \\
\text { minimization, otherwise } \\
\text { all spiked matrices } \\
\text { must be isolated and } \\
\text { treated as mixed } \\
\text { regulated waste without } \\
\text { a disposal path. Also, } \\
\text { the level of QC spike } \\
\text { suggested is above the } \\
\text { calibration levels of } \\
\text { the GC. }\end{array}$ \\
\hline $\begin{array}{l}\text { Section } 8.3 .1: \text { A } 1 \mathrm{~mL} \\
\text { volume of the (QC) } \\
\text { concentrate spiked into } \\
1 \mathrm{~L} \text { of organic free } \\
\text { water will result in a } \\
\text { sampte concentration of } \\
10-50 \mu \mathrm{g} / \mathrm{L} \text {. }\end{array}$ & $\begin{array}{l}\text { See step } 9.1 .5 .2 \text { of } \\
\text { LA-523-115: If not, add } \\
\text { the water diluent first, } \\
\text { so the sample plus } \\
\text { diluent equals } 100 \mathrm{~mL} \text {. }\end{array}$ & Deviation & $\begin{array}{l}\text { Custom semi-micro } \\
\text { liquid-l iquid extractor } \\
\text { used in LA-523-115 } \\
\text { requires less volume } \\
\text { (ca. } 100 \mathrm{~mL} \text { ) ALARA } \\
\text { concerns and limited } \\
\text { sample and hood size } \\
\text { make smaller extraction } \\
\text { vessel a necessity. }\end{array}$ \\
\hline $\begin{array}{l}8081 \mathrm{~A} \text { and } 8082 \text { are } \\
\text { separate methods. }\end{array}$ & $\begin{array}{l}8081 \mathrm{~A} \text { and } 8082 \text { have been } \\
\text { combined. }\end{array}$ & Two methods combined. & $\begin{array}{l}\text { sulfuric acid cleanup } \\
\text { limits pesticide } \\
\text { applicability. Acid } \\
\text { cleanup rarely used for } \\
\text { pesticides. }\end{array}$ \\
\hline
\end{tabular}

${ }^{8}$ NOTE: See LA-523-115 and LA-523-138 for sample preparation comparisons. 
HNF-SD-WM-DP-306, REV. 0

CLP-LIKE FORMS

20 
HNF-SD-HM-DP-306, REV. 0 $\because$

THIS PAGE INTENTIONALLY LEFT BLANK 
Data File: /chem/gc2.i/052198.b/031f0401.d HNF-SD-WM-DP-306 REV. 0 Report Date: 29-May-1998 13:25

$$
\begin{aligned}
& \text { 222-S Laboratory } \\
& \text { TARGET COMPOUNDS }
\end{aligned}
$$

Client Name:

Lab Smp Id: S98S000102

Sample Location:

Sample Date:

Sample Matrix: SoIL

Analysis Type: PEST

Data Type: GC MULTI COMP

Misc Info: $982 \mathrm{Tk} 103 / 100$
Client SDG: 052198

Client Smp ID: 982Tk103/100

Sample Point:

Date Received:

Quant Type: ESTD

Level: LOW

Operator: Gerald Ross

CONCENTRATION UNITS:

$Q$

CAS NO.

COMPOUND

(ug/L or ug/KG) ug/Kg

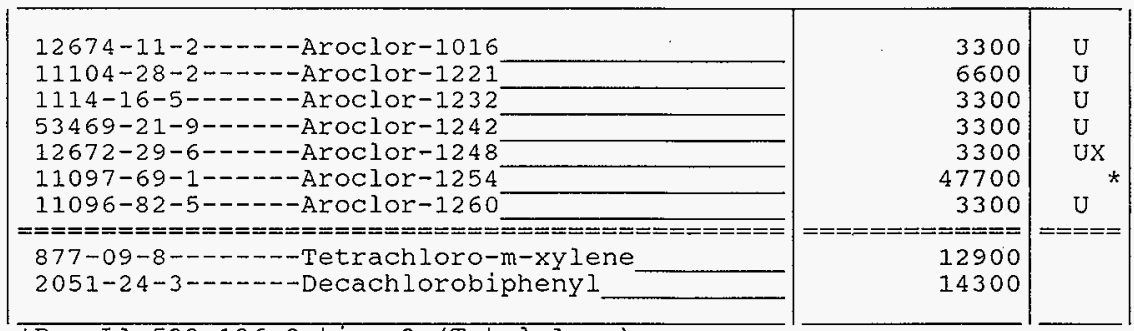

*Per LA-523-136 Option 2 (Total Area) 
Data File: /chem/gc2.i/052198.b/033f0401.d

Report Date: 29-May-1998 13:25

$$
\begin{aligned}
& \text { 222-S Laboratory } \\
& \text { TARGET COMPOUNDS }
\end{aligned}
$$

Client Name:

Lab Smp Id: $5985000102 \mathrm{D}$

Sample Location:

Sample Date:

Sample Matrix: SOIL

Analysis Type: PEST

Data Type: GC MULTI COMP

Misc Info: $984 \mathrm{Tk} 103 / 100$
Client SDG: 052198

Client Smp ID: 984Tk103/100

Sample point:

Date Received:

Quant Type: ESTD

Level: LOW

Operator: Gerald Ross

\section{CONCENTRATION UNITS:}

CAS NO. COMPOUND
( $\mathrm{ug} / \mathrm{L}$ or $\mathrm{ug} / \mathrm{KG}$ ) $\mathrm{ug} / \mathrm{Kg}$

$Q$

\begin{tabular}{r|c|}
3300 & $\mathrm{U}$ \\
6600 & $\mathrm{U}$ \\
3300 & $\mathrm{U}$ \\
3300 & $\mathrm{U}$ \\
3300 & $\mathrm{UX}$ \\
44800 & \\
3300 & $\mathrm{U}$ \\
$=========$ \\
11400 \\
11700
\end{tabular}$===$

*Per LA-523-136 Option 2 (Total Area) 
SOIL PCB COMPOUNDS MATRIX SPIKE/MATRIX SPIKE DUPLICATE RECOVERY

Lab Name: $222-5$

Lab code:

Case No.:

Matrix Spike - EPA Sample No.: $\$ 985000102$
Contract:

SAS NO.:

SDG No.: 052198

Level (low/med) LOW

\begin{tabular}{|c|c|c|c|c|c|}
\hline COMPOUND & $\begin{array}{l}\text { SPIKE } \\
\text { ADDED } \\
(\mathrm{ug} / \mathrm{Kg})\end{array}$ & $\begin{array}{c}\text { SAMPLE } \\
\text { CONCENTRATION } \\
(\mathrm{ug} / \mathrm{Kg})\end{array}$ & $\begin{array}{c}\text { MS } \\
\text { CONCENTRATION } \\
(\mathrm{ug} / \mathrm{Kg})\end{array}$ & $\begin{array}{c}\text { MS } \\
\% \\
\text { REC \# } \\
\end{array}$ & $\begin{array}{c}\text { QC. } \\
\text { LIMITS } \\
\text { REC. } \\
\end{array}$ \\
\hline Aroclor -1254 & 46600 & 47700 & 81800 & 75 & $50-150$ \\
\hline
\end{tabular}

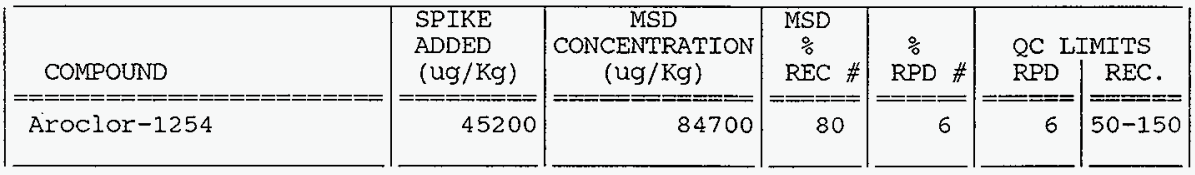

\# Column to be used to flag recovery and RPD values with an asterisk

* Values outside of QC limits

RPD: 0 out of 1 outside limits

Spike Recovery: 0 out of 2 outside limits

COMMENTS: Per LA-523-136 Option 2 (Total Area)

TABLE III PCB-2 
Data File:/chem/gc2.i/052198.b/036f0401.d

\section{2-S Laboratory \\ TARGET COMPOUNDS}

Client Name:

Lab Smp Id: S98S000102MS

Sample Location:

Sample Date:

Sample Matrix: SoIL

Analysis Type: PEST

Data Type: GC MULTI COMP

Misc Info: $986 \mathrm{Tk} 103 \mathrm{MS} / 200$
Client SDG: 052198

Client Smp ID: $986 \mathrm{Tk} 103 \mathrm{MS} / 200$

Sample Point:

Date Received:

Quant Type: ESTD

Level: LOW

Operator: Gerald Ross

CONCENTRATION UNITS:

(ug/I or $\mathrm{ug} / \mathrm{KG}$ ) ug/Kg

Q

\begin{tabular}{r|c|}
6600 & $\mathrm{U}$ \\
13200 & $\mathrm{U}$ \\
6600 & $\mathrm{U}$ \\
6600 & $\mathrm{U}$ \\
6600 & $\mathrm{UX}$ \\
81800 & ${ }^{*}$ \\
6600 & $\mathrm{U}$ \\
$=============$ \\
13600 \\
15600
\end{tabular}

* Per LA-523-136 Option 2 (Total Area) 
Data File:/chem/gc2.i/052198.b/038f0401.d

Report Date: 29-May-1998 13:25

\section{$222-s$ Laboratory}

TARGET COMPOUNDS

Client Name:

Lab Smp Id: S98S000102MSD

Sample Location:

Sample Date:

Sample Matrix: SOIL

Analysis Type: PEST

Data Type: GC MULTI COMP

Misc Info: 988Tk103MSD/200
Client SDG: 052198

Client Smp ID: $988 \mathrm{Tk} 103 \mathrm{MSD} / 200$

Sample point:

Date Received:

Quant Type: ESTD

Level: LOW

Operator: Gerald Ross

CONCENTRATION UNITS:

CAS NO. COMPOUND
( $\mathrm{ug} / \mathrm{L}$ or $\mathrm{ug} / \mathrm{KG}$ ) ug/Kg

Q

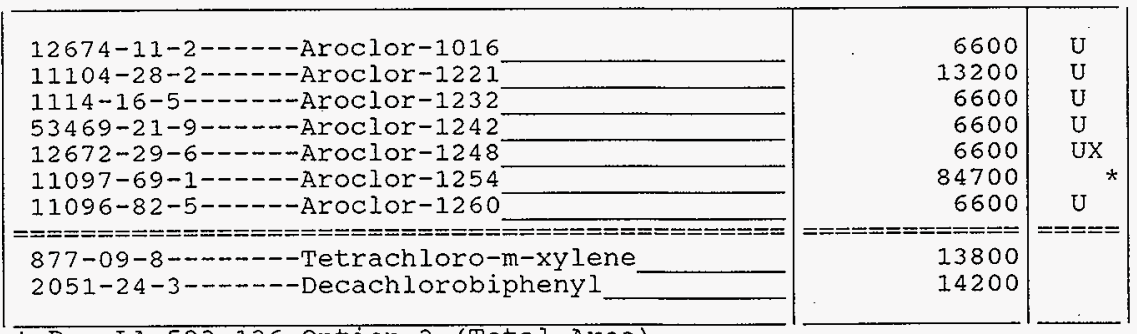

* Per LA-523-136 Option 2 (Total Area) 
HNF-SD-WM-DP-306, REV. 0

CLP-LIKE MS/MSD REPORT 
HNF-SD-WM-DP-306, REV. 0

THIS PAGE INTENTIONALLY LEFT BLANK

28 
Lab Name: 222-S

Lab code:

Case No.:

Matrix Spike - EPA Sample No.: \$98\$000102 contract:

SAS NO.:

SDG No.: 052198

Level (low/med) LOW

\begin{tabular}{|c|c|c|c|c|c|}
\hline COMPOUND & $\begin{array}{l}\text { SPIKE } \\
\text { ADDED } \\
(\mathrm{ug} / \mathrm{Kg})\end{array}$ & \begin{tabular}{|c|} 
SAMPLE \\
CONCENTRÁTION \\
(ug/Kg)
\end{tabular} & $\begin{array}{c}\text { MS } \\
\text { CONCENTRATION } \\
(\mathrm{ug} / \mathrm{Kg})\end{array}$ & $\begin{array}{l}\text { MS } \\
\% \\
\text { REC \# }\end{array}$ & $\begin{array}{l}\text { QC. } \\
\text { LIMITS } \\
\text { REC. }\end{array}$ \\
\hline Aroclor-1254 & 46600 & 47700 & 81800 & 75 & $50-150$ \\
\hline
\end{tabular}

\begin{tabular}{|c|c|c|c|c|c|c|}
\hline & $\begin{array}{l}\text { SPIKE } \\
\text { ADDED }\end{array}$ & $\begin{array}{c}\text { MSD } \\
\text { CONCENTRATION }\end{array}$ & $\begin{array}{c}\text { MSD } \\
\frac{\circ}{6}\end{array}$ & $\%$ & \multicolumn{2}{|c|}{ QC LIMITS } \\
\hline COMPOUND & $(\mathrm{ug} / \mathrm{Kg})$ & $(\mathrm{ug} / \mathrm{Kg})$ & $\mathrm{REC} \#$ & RPD \# & RPD & REC. \\
\hline Aroclor -1254 & 45200 & 84700 & 80 & 6 & 6 & $50-150$ \\
\hline
\end{tabular}

\# Column to be used to flag recovery and RPD values with an asterisk

* Values outside of QC limits

RPD: 0 out of 1 outside limits

Spike Recovery: 0 out of 2 outside limits

COMMENTS: Per LA-523-136 Option 2 (Total Area) 
HNF-SD-HM-DP-306, REV. 0

EXTRACTION BENCHSHEET 
HNF-SD-HM-DP-306, REV. 0
$\vdots$

...

THIS PAGE INTENTIONALLY LEFT BLANK

31 


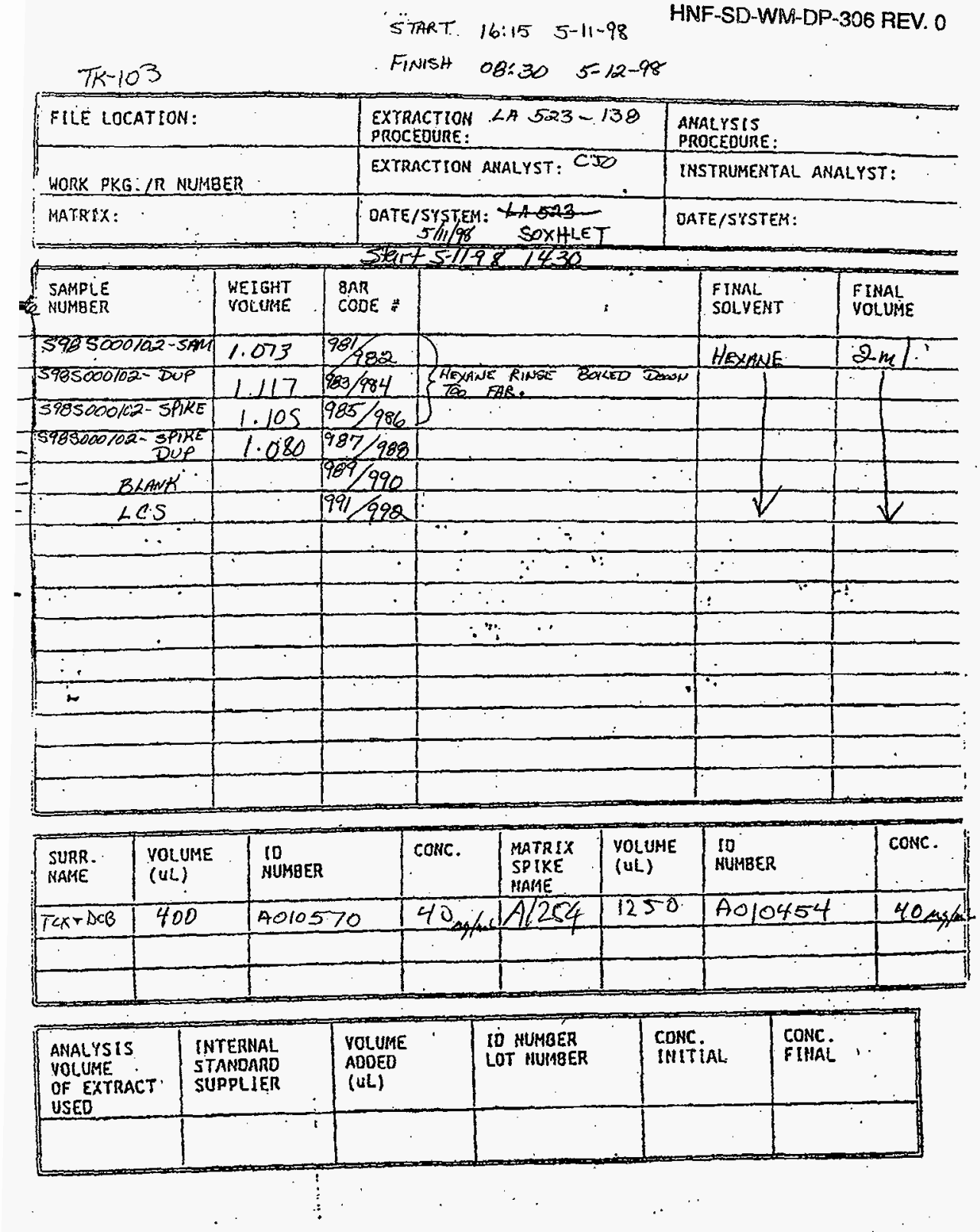


HNF-SD-WM-DP-306, REV. 0

GC SEQUENCE

33 
HNF-SD-WM-DP-306, REV. 0

$\because$

THIS PAGE INTENTIONALLY LEFT BLANK

: 
22 May $98 \quad 12: 57$ PM

Sequence: $C: \backslash H P C H E M \backslash 2 \backslash S E Q U E N C E \backslash 052198 A . S E Q$ HNF-SD-WN-DP-306 REV. 0

$\begin{array}{ll}\text { Vial Sample Name } & \text { Sample } \\ \text { Num. } & \text { Amount }\end{array}$

$200 \mathrm{ng} / \mathrm{mi}$ A1660

$200 \mathrm{ng} / \mathrm{ml}$ A1254

900TK104/1005072

I. BLNK

902TK104/1005072

I. BLNK

200ng/mI A1254

921TK102/1005060

I. BLNK

923TKI02/1005060

I. BLNK

200ingfaml-AI254

$2000 \mathrm{ng} / \mathrm{ml}$ A1254

$400 \mathrm{ng} / \mathrm{mI}$ Al254

$200 \mathrm{ng} / \mathrm{ml}$ Al254

loong/mlA1254

5 ong/mla1254

20ng/miAl254

$400 \mathrm{ng} / \mathrm{mI}$ A 1660

$200 \mathrm{ng} / \mathrm{ml}$ A1660

$100 \mathrm{~g} / \mathrm{ml}$ A1660

$50 \mathrm{ng} / \mathrm{ml}$ A1660

$20 \mathrm{ng} / \mathrm{ml}$ A. 660

$400 \mathrm{ng} / \mathrm{ml}$ A1248

$200 \mathrm{ng} / \mathrm{ml} \quad \mathrm{A1248}$

$100 \mathrm{ng} / \mathrm{ml}$ A1248

5ong/ml A1248

20ng/mi A1248

$400 \mathrm{ng} / \mathrm{ml}$ A.1242

$200 \mathrm{ng} / \mathrm{ml}$ Al242

$100 \mathrm{ng} / \mathrm{ml}$ Al242

A.00ng/ml A1232

$200 \mathrm{ng} / \mathrm{ml}$ A1232

$100 \mathrm{ng} / \mathrm{ml}$. A1232

$800 \mathrm{ng} / \mathrm{ml}$ A1221

$400 \mathrm{ng} / \mathrm{ml}$ Al221

$200 \mathrm{ng} / \mathrm{ml}$ A1221

I.Bink

c00ng/mi Al254

$200 \mathrm{ng} / \mathrm{ml} \quad \mathrm{A} 1254$

992 LCS $/ 100$

$990 \mathrm{Mblnk} / 100$

$982 \mathrm{TkiO3} / 100$

I. Blnk

984Tki03/100

I: Bink

200ng/mi A1254

$986 \mathrm{TK} 103 \mathrm{MS} / 200$

$200 \mathrm{ng} / \mathrm{ml}$ A1660

988TK103MSD/200

$200 \mathrm{ng} / \mathrm{ml}$ A2660

200ng/ml A1254

900TK104/1005072
Sample Table

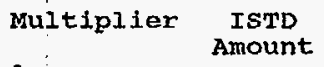

Amount 
22 May 98 i2:57 PM

Sequence: " C: \HPCHEM $\backslash 2 \backslash S E Q U E N C E \backslash 052198 A . S E Q$

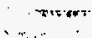

Sample Name

I. BInk

902TK104/1005072

I. BInk

$200 \mathrm{ng} / \mathrm{ml}$ Al254

921TK102/1005060

I. Blnk

923TK102/100s060

I.BInk

200 hg/mI A1254
Sample Table

Sample

Amount

Multiplier ISTD

Amount

\section{1}

1

1

1.

1

1

1

1

1

in

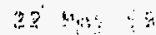

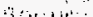


HNF-SD-WM-DP-306, REV. 0

$\because$

THIS PAGE INTENTIONALLY LEFT BLANK

38 
HNF-SD-WM-DP-306, REV. 0

INITIAL CALIBRATION REPORT

39 
HNF-SD-WNi-DP-306 REV. 0

\section{TK-103 Initial Calibration For Total Area}

Sample number $\$ 98 \$ 000102$ (5/29/98)

\begin{tabular}{|c|c|c|}
\hline Total Area & Concentration (ug/ml) of Aroclor 1254 & Ratio \\
\hline 4200793 & 400 & 10501.98 \\
\hline 2113807 & 200 & 10569.04 \\
\hline 1127555 & 100 & 11275.55 \\
\hline 513141 & 50 & 10262.84 \\
\hline 185890 & 20 & 9294.50 \\
\hline & Standard Deviation: & 714.96 \\
& Average: & 10380.78 \\
& RSD: & 0.068873 \\
& \%RSD: & 6.887321
\end{tabular}


Start Cal Date : 06-AUG-97 17:19

End Cal Date : 22-MAY-1998 20:13

Quant Method : ESTD

Origin : Force

Target Version: 3.10

Integrator

Method file : /chem/gc2.i/052198.b/rcrapcb.m

Cal Date

Curve Type

: 29-May-1998 10:43 gar

: Average

Calibration File Names:

Level 1: / chem/gc2.i/052198.b/006f0201.d

Level 2: /chem/gc2.i/052198.b/005f0202.d

Level 3:/chem/gc2.i/052198.b/004f0202.d

Level 4:/chem/gc2.i/052198.b/028f0401.d

Level 5:/chem/gc2.i/052198.b/002f0202.d

\begin{tabular}{|c|c|c|c|c|c|c|c|}
\hline & 0.0200 & 0.0500 & 0.1000 & 0.2000 & 0.4000 & & \\
\hline Compound & Level 1 & Level 2 & Level 3 & Level 4 & Level 5 & RRF & $\%$ RSD \\
\hline 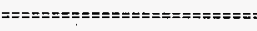 & 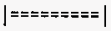 & $==\pi=x=z=$ & $== \pm= \pm==-$ & |===-====- $\mid$ & l====-==-="i & $===== \pm==1$ & 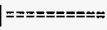 \\
\hline 1 alpha-BhC & +++++ & ++++ & ++++ & +++++ & +++++ & +++++ & $++t+t$ \\
\hline 2 beta-8HC & | +++++ & +++++ & +++++ & +++++ & +++++ & +++++ & ++++ \\
\hline 3 del ta-BHC & | +++++ & +++++ & +++++ & $++++\mid$ & $++++t$ & +++++ & +++++ \\
\hline 4 gamma-BHC (Lindane) & $++++\mid$ & +++++ & ++++ & $+4+++$ & +++++ & +++++ & +++++ \\
\hline 5 Heptachlor & ++++ & ++++ & +++++ & ++++ & $t+t+t \quad i$ & +++++ & +++++ \\
\hline 6 Aldrin & +++++ & +++++ & +++++ & +++++ & $+++++\quad \mid$ & +++++ & +++++ \\
\hline 7 Heptachlor epoxide & +++++ & +++++ & +++++ & ++++ & +++++ & ++++ & +++++ \\
\hline 8 Endosulfan 1 & +++++ & +++++ & +++++ & +++++ & +++++ & ++++ & +++++ \\
\hline 9 Dietdrin & +++++ & +++++ & ++++ & ++++ & +++++ & +++++ & +++++ \\
\hline $104,41-D D E$ & ++++ & +++++ & ++++ & $t+t+$ & ++++ & ++++ & +++++ \\
\hline 11 Endrin & $++t+t$ & +++++ & +++++ & +++++ & +++++ & +++++ & +++++ \\
\hline 12 Endosulfan II & +++++ & +++++ & +++++ & +++++ & ++++ & +++++ & ++++ \\
\hline $134,4^{\prime} \cdot 000$ & +++++ & +++++ & +++++ & ++++ & ++++ & $t+t+t$ & ++++ \\
\hline 14 Endosulfan sulfate & ++++ & ++++ & ++++ & ++++ & t++t+ & +++++ & +++++ \\
\hline $154,4^{\prime}-001$ & +++++ & +++++ & +++++ & +++++ & +++++ & $++++t$ & +++++ \\
\hline 16 Methoxychlor & +++++ & ++++ & ++++ & ++++ & ++++ & ++++ & ++++ \\
\hline 17 Endrin ketone & ++++ & ++++ & +++++ & $t+t+t$ & +++++ & ++++ & +++++ \\
\hline 18 Endrin aldehyde & ++++ & ++++ & +++++ & $+++t$ & +++++ & +++++ & +++++ \\
\hline 19 alpha-Chlordane & ++++ & +++++ & ++++ & t+t++ & $+t+t$ & ++++ & $++t+t$ \\
\hline 20 gamma-Chlordane & $t+t+$ & +++++ & ttt+t & $t+t+t$ & $++t+$ & ++++ & +++++ \\
\hline 21 Toxaphene(1) & $t+t+t$ & $t+t++$ & $++t+$ & $t+t+t$ & +++++ & ++++ & +++++ \\
\hline (2) & ++++ & $+++t$ & +++++ & ++++ & +++++ & ++++ & +++++ \\
\hline (3) & +++++ & +t+++ & +++++1 & $++++\quad 1$ & |t++t+ | & +++++ & +++++ \\
\hline 22 Aroclor $-1016(1)$ & 6850501 & $706900 i$ & 706720 & 622025 & 5134601 & $646831 \mid$ & 12.716 \\
\hline (2) & 798050 & 7742601 & 801400 & $717850 \mid$ & 592898 & 736892 & 11.830 \\
\hline (3) & $987100 \mid$ & 981500 & 1026050 & 940650 & $784878 \mid$ & 944036 & 9.955 \\
\hline 23 Aroclor $-1221(1)$ & $+++t+$ & +++++ & $222050 \mid$ & 202830 & $197182 \mid$ & 2073541 & 6.287 \\
\hline (2) & ++++ & $t++t+\quad \mid$ & 225570 & $223232 \mid$ & 240709 & $229837 \mid$ & 4.128 \\
\hline (3) & $t+t+t$ & ++++ & 5628151 & 5319051 & 537789 & 5441701 & 3.016 \\
\hline
\end{tabular}




\section{2-S Laboratory}

\section{INITIAI CALIBRATION DATA}

Start Cal Date : 06-AUG-97 $17: 19$

End Cal Date : 22-MAY-1998 20:13

Quant Method : ESTD

Origin : Force

Target Version : 3.10

Integrator : HP Genie

Method file : /chem/gc2.i/052198.b/rcrapcb.m

Cal Date : 29-May-1998 10:43 gar

Curve Type : Average

\begin{tabular}{|c|c|c|c|c|c|c|c|c|}
\hline & & 10.0200 & 0.0500 & 0.1000 & 0.2000 & 0.4000 & 1 & \\
\hline & Compound & | Level 1 & Level 2 & Level 3 & Level 4 & Level 5 & RRF & $\%$ RSD \\
\hline \multicolumn{9}{|c|}{ 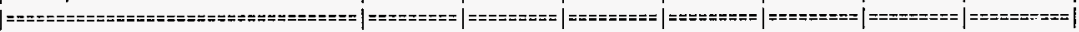 } \\
\hline & 24 Aroctor $-1232(1)$ & +++++ & +++++ & 549340 & 559235 & 460210 & 522928 & $10.430 \mid$ \\
\hline & (2) & +++++ & +++++ & 3268701 & 350830 & 292162 & $323288 i$ & 9.124 \\
\hline & (3) & ++++ & $t+t+t$ & 401730 & 437850 & 394132 & $411238]$ & 5.6801 \\
\hline & 25 Aroclor $-1242(1)$ & +++++ & $t+t+t$ & 467560 & 512610 & 456095 & $478755 j$ & $6.240 \mid$ \\
\hline & (2) & +++++ & +++++ & 509520 & 576045 & 530280 & 5386151 & $6.319 \mid$ \\
\hline & (3) & +++++ & +++++ & 625990 & 731335 & 693558 & $683628]$ & $7.807 \mid$ \\
\hline & 26 Aroctor $-1248(1)$ & 767300 & 692800 & 677830 & 720975 & 629835 & 697748 & 7.311 \\
\hline & (2) & $675800 \mid$ & $635180 \mid$ & $628660 \mid$ & 688730 & 616158 & 648906 & 4.861 \\
\hline & (3) & 9105501 & 8409201 & 8155601 & 899215 & 803228 & 853894 & $5.698 \mid$ \\
\hline & 27 Aroctor $-1254(1)$ & 8273001 & $812780 \mid$ & 8034401 & 839655 & 792698 & 815200 & $2.291 \mid$ \\
\hline & (2) & 1101500 & 1086100 & $1060040 \mid$ & 1086780 & 1057750 & $1078434]$ & 1.751 \\
\hline & (3) & 9360001 & 941120 & 936560 & 1003545 & 1020202 & 9674861 & 4.237 \\
\hline & 28 Aroclor $-1260(1)$ & 1056950 & $1017260 \mid$ & 1040630 & 979115 & $775998 i$ & 9739901 & 11.754 \\
\hline & (2) & $1060000 \mid$ & 994400 & 10349301 & 982735 & $769212 i$ & 968256 & 11.9301 \\
\hline & (3) & 860200 & 901620 & $960980 \mid$ & 957400 & 7538301 & 886806 & $9.617 \mid$ \\
\hline \multicolumn{9}{|c|}{ 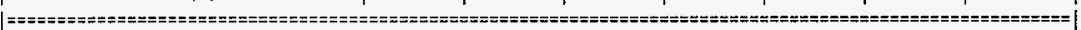 } \\
\hline \$ $\$$ & 29 Tetrachloro-m-xylene & $14389700 \mid$ & $14672520 \mid$ & 16410640 & $14254860 \mid$ & $|13998965|$ & 14745337 & $6.525 \mid$ \\
\hline$\$$ & 30 Decachlorobiphenyl & 8633100 & 8030800 & 8248180 & 7121710 & $|8643725|$ & 8135503 & $7.670 \mid$ \\
\hline
\end{tabular}


HNF-SD-HM-DP-306, REV. 0

RAW DATA 
HNF-SD-WM-DP-306, REV. 0

THIS PAGE INTENTIONALLY LEFT BLANK 
Data File: /chem/gc2.i/052198.b/002f0202.d Report Date: 28-May-1998 11:07

Page 1

HNF-SD-WM-DP-306 REV. 0

222-S Laboratory

Data file:/chem/gc2.i/052198.b/002f0202.d

Lab Smp Id: 400ng/mi A1254

Inj Date : 21-MAY-98 12:44

Operator : Gerald Ross

Smp Info : $400 \mathrm{ng} / \mathrm{ml} \mathrm{A1254}$

Misc Info: $400 \mathrm{ng} / \mathrm{ml} \mathrm{A1254}$

comment :

Method : /chem/gc2.i/052198.b/rcrapcb.m

Meth Date : 28-May-1998 11:07

Cal Date : 21-MAY-98 12:44

Als bottle: 1

Dil Factor: 1.000

Integrator: HP Genie

Target Version: 3.10

\begin{tabular}{cr}
$R T$ & \multicolumn{1}{l}{$A R E A$} \\
$=============$ \\
10.633 & 29610 \\
11.743 & 26535 \\
12.659 & 2799793 \\
13.021 & 10280 \\
13.702 & 30007 \\
17.700 & 10917 \\
18.545 & 21510 \\
18.694 & 194153 \\
18.915 & 46461 \\
19.700 & 95866 \\
20.225 & 60615 \\
21.370 & 31716 \\
21.532 & 143965 \\
21.773 & 317079 \\
22.106 & 43922 \\
22.563 & 86826 \\
22.852 & 423101 \\
23.136 & 126105 \\
23.736 & 20162 \\
24.004 & 109825 \\
24.268 & 207110 \\
24.515 & 56066 \\
24.788 & 408081 \\
25.420 & 80811 \\
25.636 & 57860 \\
25.846 & 26090 \\
26.109 & 438219 \\
26.646 & 24059 \\
26.934 & 42045 \\
27.260 & 167145 \\
27.571 & 224254 \\
27.776 & 11031 \\
&
\end{tabular}

HEIGHT
$========$
2346
1501
155665
590
1599
300
916
8322
1998
4115
1559
1302
5771
11987
1797
3024
14871
5146
803
4315
7932
2261
14566
2726
2068
1013
10467
782
1318
6311
5914
413

0.079

0.057

0.056

0.057

0.053

0.027

0.043

0.043

0.043

0.043

0.026

0.041

0.040

0.038

0.041

0.035

0.035

0.041

0.040

0.039

0.038

0.040

0.036

0.034

0.036

0.039

0.024

0.033

0.031

0.038

0.026

0.037

Client Smp ID: $400 \mathrm{ng} / \mathrm{ml}$ A1254

Inst ID: $g C 2 . i$

Quant Type: AREA\%

Cal File: $002 f 0202$,d

Calibration Sample, Level: 5

Compound Sublist: AR1254.sub

Sample Matrix: WATER

HT/AREA \% AREA

0.339

0.304

32.1

0.118

0.344

0.125

0.246

2.22

0.532

1.10

0.694

0.363

1.65

3.63

0.503

0.995

4.85

1.44

0.231

1.26

2.37

0.642

4.67

0.926

0.663

0.299

5.02

0.276

0.482

1.91

2.57

0.126
Compounds

Tetrachloro-m-xylene

Aroclor-1254

Aroclor -1254

Aroclor-1254 
HNF-SD-MYM-DP-306 REV. 0

Data File: /chem/gc2.i/052198.b/002f0202.d Report Date: 28-May-1998 11:07

\begin{tabular}{|c|c|c|c|c|c|}
\hline $\begin{array}{c}\mathrm{RT} \\
=====\end{array}$ & $\begin{array}{c}\text { AREA } \\
=========\end{array}$ & $\begin{array}{c}\text { HEIGHT } \\
=========\end{array}$ & $\begin{array}{l}\mathrm{HT} / \mathrm{AREA} \\
======\end{array}$ & $\begin{array}{l}\% \text { AREA } \\
======\end{array}$ & 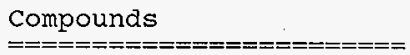 \\
\hline 27.982 & 44231 & 1760 & 0.040 & 0.507 & \\
\hline 28.380 & 19920 & 772 & 0.039 & 0.228 & \\
\hline 28.520 & 23048 & 848 & 0.037 & 0.264 & \\
\hline 28.810 & 279379 & 9637 & 0.034 & 3.20 & \\
\hline 28.958 & 37673 & 1431 & 0.038 & 0.432 & \\
\hline 29.286 & 21948 & 760 & 0.035 & 0.251 & \\
\hline 29.688 & 12730 & 453 & 0.036 & 0.146 & \\
\hline 30.365 & 76061 & 2465 & 0.032 & 0.871 & \\
\hline 31.008 & 13807 & 499 & 0.036 & 0.158 & \\
\hline 31.604 & 38731 & 1318 & 0.034 & 0.444 & \\
\hline 32.469 & 30323 & 992 & 0.033 & 0.347 & \\
\hline 34.154 & 20924 & 732 & 0.035 & 0.240 & \\
\hline 40.340 & 10592 & 288 & 0.027 & 0.121 & \\
\hline 42.741 & 1.728 .745 & 38902 & 0.023 & 19.8 & Decachlorobiphenyl \\
\hline
\end{tabular}

Total area $=8729331$

$-2,799,793$

$-1,728,745$

$4,200,793$ 
Data File: /chem/gc2.i/052198.b/028f0401.d

Calfile

Report Date: 28-May-1998 11:08

\section{2-S Laboratory}

Data file:/chem/gc2.i/052198.b/028f0401.d

Lab Smp Id: $200 \mathrm{ng} / \mathrm{ml}$ A1254

Inj Date : 22-MAY-98 20:13

Operator : Gerald Ross

Smp Info : 200ng/ml A1254

Client Smp ID: $200 \mathrm{ng} / \mathrm{ml}$ A1254

Misc Info: $200 \mathrm{ng} / \mathrm{ml} \mathrm{A1254}$

Inst ID: $g \subset 2 \cdot i$

comment

Method

/chem/gc2.i/052198.b/rcrapcb.m

Meth Date : 28-May-1998 11:08

cal Date : 22-MAY-98 20:13

Als bottle: 1

Dil Factor: 1.000

Integrator: HP Genie

Target Version: 3.10

$\begin{array}{lr}\text { RT } & \text { AREA } \\ ==============1 \\ 12.654 & 1425487 \\ 13.697 & 14256 \\ 17.694 & 12011 \\ 18.538 & 10860 \\ 18.688 & 101253 \\ 18.908 & 24102 \\ 19.693 & 48378 \\ 20.286 & 13523 \\ 21.364 & 16701 \\ 21.527 & 72478 \\ 21.767 & 167932 \\ 22.098 & 23682 \\ 22.556 & 44208 \\ 22.846 & 217356 \\ 23.128 & 63252 \\ 23.997 & 54109 \\ 24.261 & 100067 \\ 24.508 & 27401 \\ 24.780 & 200709 \\ 25.411 & 39729 \\ 25.627 & 28286 \\ 25.838 & 12326 \\ 26.101 & 215530 \\ 26.637 & 12271 \\ 26.926 & 20827 \\ 27.252 & 82374 \\ 27.561 & 107489 \\ 27.971 & 20255 \\ 28.372 & 10869 \\ 28.511 & 13180 \\ 28.802 & 18330 \\ 28.951 & \\ & \end{array}$

\begin{tabular}{|c|c|c|}
\hline $\begin{array}{c}\text { HEIGHT } \\
=========\end{array}$ & $\begin{array}{l}\mathrm{HT} / \mathrm{AREA} \\
=====\end{array}$ & $\begin{array}{l}\% \text { AREA } \\
======\end{array}$ \\
\hline 79944 & 0.056 & 33. \\
\hline 741 & 0.052 & 0.33 \\
\hline 181 & 0.015 & 0.282 \\
\hline 474 & 0.044 & 0.255 \\
\hline 4350 & 0.043 & 2.38 \\
\hline 1013 & 0.042 & 0.567 \\
\hline 2059 & 0.043 & 1.14 \\
\hline 552 & 0.041 & 0.318 \\
\hline 658 & 0.039 & 0.393 \\
\hline 2843 & 0.039 & 1.70 \\
\hline 6280 & 0.037 & 3.95 \\
\hline 927 & 0.039 & 0.557 \\
\hline 1508 & 0.034 & 1.04 \\
\hline 7630 & 0.035 & 5.11 \\
\hline 2548 & 0.040 & 1.49 \\
\hline 2133 & 0.039 & 1.27 \\
\hline 3786 & 0.038 & 2.35 \\
\hline 1073 & 0.039 & 0.644 \\
\hline 7085 & 0.035 & 4.72 \\
\hline 1333 & 0.034 & 0.934 \\
\hline 1008 & 0.036 & 0.665 \\
\hline 482 & 0.039 & 0.290 \\
\hline 5111 & 0.024 & 5.07 \\
\hline 394 & 0.032 & 0.289 \\
\hline 651 & 0.031 & 0.490 \\
\hline 3110 & 0.038 & 1.94 \\
\hline 2746 & 0.026 & 2.53 \\
\hline 802 & 0.040 & 0.476 \\
\hline 411 & 0.038 & 0.256 \\
\hline 448 & 0.034 & 0.310 \\
\hline 4638 & 0.034 & 3.20 \\
\hline 692 & 0.038 & 0 \\
\hline
\end{tabular}

Compounds

$==-==1$
Tetrachloro-m-xylene

Aroclor -1254

Aroclor -1254

Aroclor-1254 


\section{HNF-SD-WRi-DP-306 REV. 0}

Data File: /chem/gc2.i/052198.b/028f0401.d Report Date: 28-May-1998 11:08

Page 2

\begin{tabular}{|c|c|c|c|c|c|}
\hline$\stackrel{\mathrm{RT}}{==}=$ & $=\stackrel{\text { AREA }}{=====-==}$ & $\begin{array}{c}\text { HEIGHT } \\
==== \pm=====\end{array}$ & $\begin{array}{l}\text { HT / AREA } \\
======\end{array}$ & $\begin{array}{l}\because \text { AREA } \\
=====\end{array}$ & $\begin{array}{l}\text { Compounds } \\
==================1=0\end{array}$ \\
\hline 29.276 & 10400 & 364 & 0.035 & 0.245 & \\
\hline 30.356 & 35339 & 1149 & 0.033 & 0.831 & \\
\hline 31.596 & 17979 & 611 & 0.034 & 0.423 & \\
\hline 32.463 & 17886 & 494 & 0.028 & 0.421 & \\
\hline 36.494 & 53724 & 176 & 0.003 & 1.26 & \\
\hline 37.804 & 29087 & 231 & 0.008 & 0.684 & \\
\hline 38.587 & 19490 & 95 & 0.005 & 0.458 & \\
\hline 42.728 & 712171 & 16203 & 0.023 & 16.8 & Decachlorobiphenyl \\
\hline
\end{tabular}

Total area $=4251465$

$$
\begin{aligned}
& -142548 \\
& \because 1=171 \\
& 2,113,807
\end{aligned}
$$


Data File: /chem/gc2.i/052198.b/004f0202.d

Report Date: 28-May-1998 11:07

Page 1

\section{2-S Laboratory}

Data file:/chem/gc2.i/052198.b/004f0202.d

Lab Smp Id: $100 \mathrm{ng} / \mathrm{ml}$ A1254

Inj Date : 21-MAY-98 17:41

operator : Gerald Ross

Smp Info : $100 \mathrm{ng} / \mathrm{ml}$ A1254

Misc Info : $100 \mathrm{ng} / \mathrm{ml}$ A1254

Comment

Method

Meth Date : 28-May-1998 11:07

Cal Date : 21-MAY-98 17:41

Als bottle: 1

Dil Factor: 1.000

Integrator: HP Genie

Target Version: 3.10

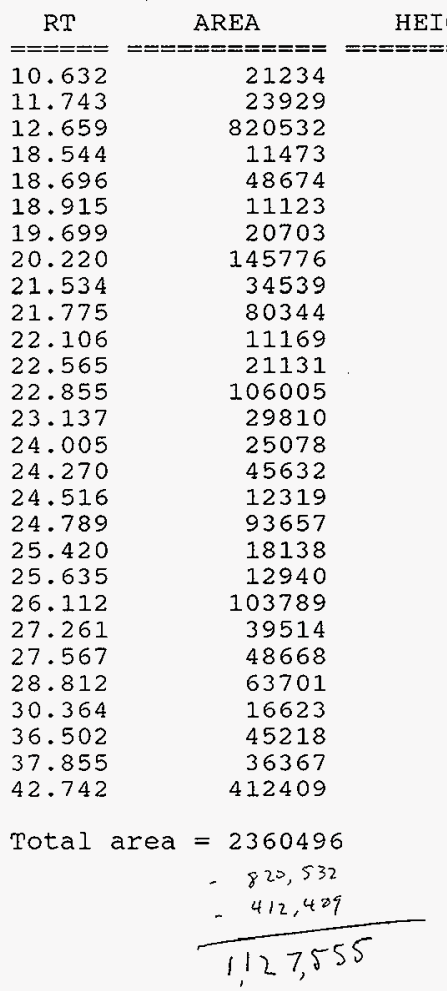

Client Smp ID: 100ng/ml A1254

Inst ID: $\mathrm{gC} 2 . \mathrm{i}$ m

Quant Type: AREA\%

Cal File: 004f0202.d

Calibration Sample, Level: 3

Compound sublist: AR1254.sub

Sample Matrix: WATER
HT/AREA \% AREA Compounds

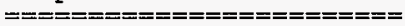
1671

1218

47207

480

2052

458

908

6193

1336

3013

429

690

3757

1196

989

1710

486

3305

611

460

2476

1490

1258

2194

528

113

484

9504
0.079

0.051

0.058

0.042

0.042

0.041

0.044

0.042

0.039

0.038

0.038

0.033

0.035

0.040

0.039

0.037

0.039

0.035

0.034

0.036

0.024

0.038

0.026

0.034

0.032

0.002

0.013

0.023
0.900

1.01

34.8

0.486

2.06

0.471

0.877

6.18

1.46

3.40

0.473

0.895

4.49

1.26

1.06

1.93

0.522

3.97

0.768

0.548

4.40

1.67

2.06

2.70

0.704

1.92

1.54

17.5
Tetrachloro-m-xylene

Aroclor -1254

Aroclor -1254

Aroclor -1254

Decachlorobiphenyl 


\section{2-S Laboratory}

Data file:/chem/gc2.i/052198.b/005f0202.d
Lab Smp Id: 50ng/mi A1254
Client Smp ID: 50ng/mi A1254
Inj Date : 21-MAY-98 19:34
operator : Gerald Ross
Simp Info: $50 \mathrm{ng} / \mathrm{ml} \mathrm{A} 1254$
Inst ID: $g \mathrm{~g} 2 \cdot \dot{i}$
Misc Info : $50 \mathrm{ng} / \mathrm{ml} \mathrm{Al254}$
Comment :
Method : /chem/gc2.i/052198.b/rcrapcb.m
Meth Date : 28-May-1998 11:07
Cal Date : 21-MAY-98 19:34
Als bottle: 1
Dil Factor: 1.000
Integrator: HP Genie
Target Version: 3.10
Quant Type: AREA\%
Cal File: 005 f0202.d
Calibration Sample, Level: 2
Compound Sublist: AR1254.sub
Sample Matrix: WATER

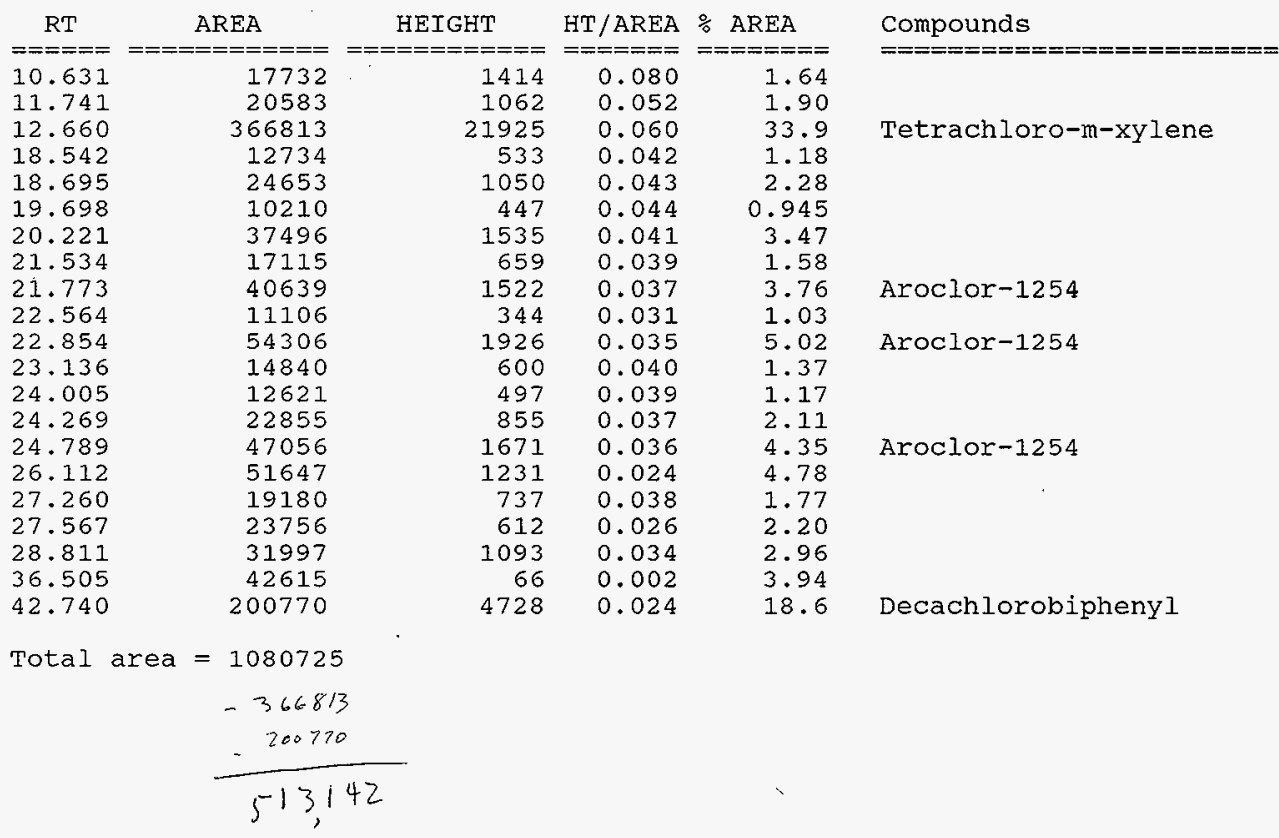


Data File: /chem/gc2.i/052198.b/006f0201.d

\section{2-S Laboratory}

Data file:/chem/gc2.i/052198.b/006f0201.d

Lab Smp Id: 20ng/ml A 1254

Inj Date : 21-MAY-98 20:31

operator : Gerald Ross

Smp Info : $20 \mathrm{ng} / \mathrm{ml} \mathrm{A} 1254$

Client Smp ID: $20 \mathrm{ng} / \mathrm{ml}$ A1254

Misc Info : $20 \mathrm{ng} / \mathrm{ml} \mathrm{A} 1254$

Comment :

Method : /chem/gc2.i/052198.b/rcrapcb.m

Meth Date : 28-May-1998 11:07

Cal Date : 21-MAY-98 20:31.

Als bottle: 1

Dil Factor: 1.000

Integrator: HP Genie

Target Version: 3.10

Inst ID: $g c 2 . i$

\begin{tabular}{|c|c|c|c|c|}
\hline $\begin{array}{c}\mathrm{RT} \\
=====\end{array}$ & $==\stackrel{\text { AREA }}{==}==$ & $\begin{array}{c}\text { HEIGHT } \\
========-=\end{array}$ & $\begin{array}{l}\mathrm{HT} / \mathrm{AREA} \\
======\end{array}$ & $\begin{array}{l}\% \text { AREA } \\
======\end{array}$ \\
\hline 10.631 & 14378 & 1195 & 0.083 & 2 \\
\hline 11.741 & 18434 & 920 & 0.050 & \\
\hline 12.660 & 143897 & 8605 & 0.060 & \\
\hline 18.542 & 10495 & 438 & 0.042 & \\
\hline 18.695 & 10217 & 423 & 0.041 & \\
\hline 20.220 & 22094 & 923 & 0.042 & 4 \\
\hline 21.774 & 16547 & 617 & 0.037 & \\
\hline 22.854 & 22030 & 781 & 0.035 & \\
\hline 24.788 & 18721 & 656 & 0.035 & \\
\hline 26.113 & 19964 & 474 & 0.024 & \\
\hline 28.810 & 10418 & 396 & 0.038 & \\
\hline 36.506 & $67060-$ & 45 & 0.001 & \\
\hline 37.841 & 24592 & 316 & 0.013 & \\
\hline 42.737 & 86332 & 2024 & 0.023 & 17 \\
\hline
\end{tabular}

Compounds

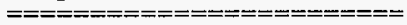

Tetrachloro-m-xylene

Aroclor -1254

Aroclor -1254

Aroclor -1254

4.11

2.15

5.07

17.8

Decachlorobipheny 1

Total area $=485179$

$$
\begin{aligned}
& .143897 \\
& \begin{array}{r}
86332 \\
=63060
\end{array} \\
& 25-4950-4725-29-98 \\
& 185,890
\end{aligned}
$$


Data File: /chem/gc2.j/052198.b/007f0302.d

other calfiles

Report Date: 28-May-1998 11:07

Page 1

\section{2-S Laboratory}

Data file:/chem/gc2.i/052198.b/007f0302.d

Lab Smp Id: 400ng/ml A 1660

Inj Date : 21-MAY-98 23:21

operator : Gerald Ross

Smp Info : 400ng/mi A1660

Misc Info: $400 \mathrm{ng} / \mathrm{ml}$ A1 660

Comment :

Method : /chem/gc2.i/052198.b/rcrapcb.m

Meth Date : 28-May-1998 11:07

Cal Date : 21-MAY-98 23:21

Als bottle: 1

Dil Factor: 1.000

Integrator: HP Genie

Target Version: 3.10

\begin{tabular}{|c|c|c|c|c|}
\hline$==\begin{array}{c}\mathrm{RT} \\
===\end{array}$ & $\begin{array}{c}\text { AREA } \\
=========\end{array}$ & $\begin{array}{c}\text { HEIGHT } \\
==========\end{array}$ & $\begin{array}{l}\mathrm{HT} / \mathrm{AREA} \\
=====\end{array}$ & $\begin{array}{l}\% \text { AREA } \\
=====\end{array}$ \\
\hline 10.629 & 13014 & 1035 & 0.080 & 0.1 \\
\hline 11.738 & 15258 & 877 & 0.057 & 0.15 \\
\hline 12.544 & 17164 & 1164 & 0.068 & 0.17 \\
\hline 12.654 & 2371972 & 131100 & 0.055 & 24 \\
\hline 13.284 & 24282 & 1027 & 0.042 & 0.25 \\
\hline 13.651 & 21341 & 1562 & 0.073 & 0.21 \\
\hline 13.687 & 32480 & 1676 & 0.052 & 0.33 \\
\hline 13.851 & 136246 & 6592 & 0.048 & 1.4 \\
\hline 14.609 & 24053 & 1164 & 0.048 & 0.24 \\
\hline 15.370 & 205385 & 10159 & 0.049 & 2.1 \\
\hline 15.468 & 132821 & 5495 & 0.041 & 1.3 \\
\hline 15.795 & 21642 & 836 & 0.039 & 0.22 \\
\hline 16.157 & 170224 & 6770 & 0.040 & 1.7 \\
\hline 16.804 & 49941 & 2341 & 0.047 & 0.51 \\
\hline 16.934 & 26417 & 1195 & 0.045 & 0.27 \\
\hline 17.164 & 237160 & 12206 & 0.051 & 2.4 \\
\hline 17.241 & 313952 & 13537 & 0.043 & 3.2 \\
\hline 17.675 & 217888 & 9130 & 0.042 & 2.2 \\
\hline 18.022 & 121738 & 5234 & 0.043 & 1.2 \\
\hline 18.271 & 46831 & 1994 & 0.043 & 0.48 \\
\hline 18.686 & 164385 & 6536 & 0.040 & 1.6 \\
\hline 18.905 & 120769 & 5364 & 0.044 & 1.2 \\
\hline 19.104 & 98879 & 2609 & 0.026 & 1.0 \\
\hline 19.689 & 155107 & 6609 & 0.043 & 1.5 \\
\hline 19.878 & 94100 & 3022 & 0.032 & 0.96 \\
\hline 20.218 & 75368 & 3624 & 0.048 & 0.77 \\
\hline 20.291 & 101848 & 3740 & 0.037 & 1.0 \\
\hline 20.716 & 24822 & 1048 & 0.042 & 0.25 \\
\hline 21.363 & 15159 & 600 & 0.040 & 0.15 \\
\hline 21.527 & 26030 & 1018 & 0.039 & 0.26 \\
\hline 21.765 & 137441 & 5224 & 0.038 & 1.4 \\
\hline 22.557 & 22016 & 720 & 0.033 & 0.22 \\
\hline
\end{tabular}

Client Smp ID: $400 \mathrm{ng} / \mathrm{ml}$ A1660

Inst ID: $9 \subset 2 . i$

Quant Type: AREA\%

Cal File: 007f0302.d

Calibration Sample, Level: 5

Compound Sublist: AR1660.sub

Sample Matrix: WATER

Compounds

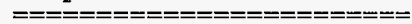

Tetrachloro-m-xylene

Aroclor -1016

Aroclor -1016

Aroclor -1016 
HNF-SD-WM-DP-306 REV. 0

Data File:/Chem/gc2.i/052198.b/007f0302.d Report Date: 28-May-1998 11:07

\begin{tabular}{|c|c|c|c|c|c|}
\hline$====$ & $\begin{array}{c}\text { AREA } \\
=========\end{array}$ & $\begin{array}{c}\text { HEIGHT } \\
==========\end{array}$ & $\begin{array}{l}\text { HT / AREA } \\
======\end{array}$ & $\begin{array}{l}\% \text { AREA } \\
=======\end{array}$ & 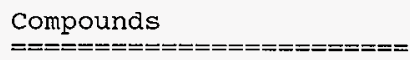 \\
\hline 22.839 & 135251 & 5248 & 0.039 & 1.39 & \\
\hline 24.262 & 19150 & 756 & 0.039 & 0.197 & \\
\hline 24.685 & 59543 & 2393 & 0.040 & 0.612 & \\
\hline 24.779 & 57954 & 2238 & 0.039 & 0.595 & \\
\hline 25.390 & 136198 & 5415 & 0.040 & 1.40 & \\
\hline 25.625 & 77047 & 2730 & 0.035 & 0.792 & \\
\hline 26.015 & 310399 & 10847 & 0.035 & 3.19 & Aroclor-1260 \\
\hline 26.636 & 19303 & 658 & 0.034 & 0.198 & \\
\hline 26.927 & 48310 & 1785 & 0.037 & 0.496 & \\
\hline 27.249 & 307686 & 11528 & 0.037 & 3.16 & Aroclor -1260 \\
\hline 27.522 & 101088 & 3699 & 0.037 & 1.04 & \\
\hline 27.970 & 87037 & 3761 & 0.043 & 0.894 & \\
\hline 28.132 & 59981 & 2499 & 0.042 & 0.616 & \\
\hline 28.518 & 32904 & 1239 & 0.038 & 0.338 & \\
\hline 28.797 & 301532 & 10229 & 0.034 & 3.10 & Aroclor -1260 \\
\hline 28.948 & 29449 & 1162 & 0.039 & 0.302 & \\
\hline 29.231 & 39452 & 1368 & 0.035 & 0.405 & \\
\hline 29.677 & 181508 & 6628 & 0.037 & 1.86 & \\
\hline 29.957 & 89906 & 3325 & 0.037 & 0.924 & \\
\hline 30.351 & 24636 & 734 & 0.030 & 0.253 & \\
\hline 30.588 & 22861 & 860 & 0.038 & 0.235 & \\
\hline 30.995 & 159815 & 5849 & 0.037 & 1.64 & \\
\hline 31.310 & 88318 & 3218 & 0.036 & 0.907 & \\
\hline 31.570 & 67841 & 1833 & 0.027 & 0.697 & \\
\hline 31.908 & $11034^{\circ}$ & 415 & 0.038 & 0.113 & \\
\hline 32.093 & 22269 & 865 & 0.039 & 0.229 & \\
\hline 32.453 & 310283 & 10960 & 0.035 & 3.19 & \\
\hline 32.602 & 17855 & 702 & 0.039 & 0.183 & \\
\hline 34.139 & 140932 & 4485 & 0.032 & 1.45 & \\
\hline 34.566 & 63008 & 2286 & 0.036 & 0.647 & \\
\hline 34.873 & 72205 & 2415 & 0.033 & 0.742 & \\
\hline 36.625 & 27262 & 862 & 0.032 & 0.280 & \\
\hline 37.748 & 68754 & 1967 & 0.029 & 0.706 & \\
\hline 40.325 & 26176 & 712 & 0.027 & 0.269 & \\
\hline 42.723 & 1279516 & 29251 & 0.023 & 13.1 & Decachlorobiphenyl \\
\hline
\end{tabular}

Total area $=9732198$ 
Data File: /chem/gc2,i/052198.b/009f0401.d

Report Date: 28-May-1998 11:07

Page 1

\section{2-S Laboratory}

Data file:/chem/gc2.i/052198.b/009f0401.d
Lab Smp Id: 100ng/ml Al660
Inj Date : 22-MAY-98 02:11
operator : Gerald Ross
Smp Info : 100ng/ml A1660
Client Smp ID: $100 \mathrm{ng} / \mathrm{ml}$ A1660
Misc Info: $100 \mathrm{ng} / \mathrm{ml}$ A1660
Comment :
Method : /chem/gc2.i/052198.b/rcrapcb.m
Meth Date : 28-MaY-1998 11:07
Cal Date : 22-MAY-98 02:11
Als bottle: 1
Dil Factor: 1.000
Integrator: HP Genie
Target Version: 3.10
Inst ID: $g c 2 . i$
Quant Type: AREA\%
Cal File: 009f0401.d
Calibration Sample, Level: 3
Compound Sublist: AR1660.sub
Sample Matrix: WATER

\begin{tabular}{|c|c|c|c|c|c|}
\hline$==\stackrel{\mathrm{RT}}{==}$ & $\begin{array}{c}\mathrm{AREA} \\
==========\end{array}$ & $\begin{array}{c}\text { HEIGHT } \\
==========x\end{array}$ & $\begin{array}{l}\mathrm{HT} / \mathrm{AREA} \\
=======\end{array}$ & $\begin{array}{l}\% \text { AREA } \\
======\end{array}$ & $\begin{array}{l}\text { Compounds } \\
===================\end{array}$ \\
\hline 10.629 & 10173 & 828 & 0.081 & 0.305 & \\
\hline 11.739 & 12920 & 735 & 0.057 & 0.387 & \\
\hline 12.655 & 878716 & 50321 & 0.057 & 26.3 & Tetrachloro-m-xylene \\
\hline 13.686 & 17692 & 549 & 0.031 & 0.530 & \\
\hline 13.854 & 46931 & 2244 & 0.048 & 1.41 & \\
\hline 15.372 & 70673 & 3466 & 0.049 & 2.12 & Aroclor-1016 \\
\hline 15.469 & 42615 & 1751 & 0.041 & 1.28 & \\
\hline 16.160 & 54886 & 2142 & 0.039 & 1.64 & \\
\hline 16.806 & 15888 & 729 & 0.046 & 0.476 & \\
\hline 17.166 & 80140 & 4080 & 0.051 & 2.40 & Aroclor-1016 \\
\hline 17.243 & 102605 & 4424 & 0.043 & 3.08 & Aroclor-1016 \\
\hline 17.678 & 75359 & 3005 & 0.040 & 2.26 & \\
\hline 18.025 & 40630 & 1594 & 0.039 & 1.22 & \\
\hline 18.271 & 16402 & 665 & 0.041 & 0.492 & \\
\hline 18.538 & 18845 & 778 & 0.041 & 0.565 & \\
\hline 18.687 & 55519 & 2205 & 0.040 & 1.66 & \\
\hline 18.906 & 39141 & 1732 & 0.044 & 1.17 & \\
\hline 19.106 & 31641 & 829 & 0.026 & 0.949 & \\
\hline 19.691 & 50336 & 2119 & 0.042 & 1.51 & \\
\hline 19.880 & 29778 & 921 & 0.031 & 0.893 & \\
\hline 20.216 & 75623 & 2048 & 0.027 & 2.27 & \\
\hline 21.767 & 45111 & 1714 & 0.038 & 1.35 & \\
\hline 22.841 & 44435 & 1713 & 0.039 & 1.33 & \\
\hline 24.685 & 18670 & 766 & 0.041 & 0.560 & - \\
\hline 24.779 & 17649 & 682 & 0.039 & 0.529 & \\
\hline 25.390 & 43558 & 1731 & 0.040 & 1.30 & \\
\hline 25.624 & 24033 & 857 & 0.036 & 0.720 & \\
\hline 26.016 & 104063 & 3750 & 0.036 & 3.12 & Aroclor -1260 \\
\hline 26.927 & 15008 & 556 & 0.037 & 0.450 & \\
\hline 27.250 & 103493 & 3907 & 0.038 & 3.10 & Aroclor -1260 \\
\hline 27.522 & 31973 & 1157 & 0.036 & 0.958 & \\
\hline 27.970 & 22640 & 1024 & 0.045 & 0.679 & \\
\hline
\end{tabular}

Compounds

$\begin{array}{lr}=====1======== \\ 10.629 & 10173 \\ 11.739 & 12920 \\ 12.655 & 878716 \\ 13.686 & 17692 \\ 13.854 & 46931 \\ 15.372 & 70673 \\ 15.469 & 42615 \\ 16.160 & 54886 \\ 16.806 & 15888 \\ 17.166 & 80140 \\ 17.243 & 102605 \\ 17.678 & 75359 \\ 18.025 & 40630 \\ 18.271 & 16402 \\ 18.538 & 18845 \\ 18.687 & 55519 \\ 18.906 & 39141 \\ 19.106 & 31641 \\ 19.691 & 50336 \\ 19.880 & 29778 \\ 20.216 & 75623 \\ 21.767 & 45111 \\ 22.841 & 44435 \\ 24.685 & 18670 \\ 24.779 & 17649 \\ 25.390 & 43558 \\ 25.624 & 24033 \\ 26.016 & 104063 \\ 26.927 & 15008 \\ 27.250 & 103493 \\ 27.522 & 31973 \\ 27.970 & 22640 \\ & \end{array}$ $===$ 828

735

549

2244

3466

1751

2142

729

4080

4424

3005

1594

665

778

2205

1732

829

2119

921

2048

1714

1713

766

682

1731

857

3750

556

1157

0.045
.387

26.3

1.41

2.12

1.28

1.64

.476

2.40

.08

2.26

1.22

0.492

0.565

1.66

0.893

2.27

1.35

1.33

0.560

0.529

1.30

3.12

0.450

3.10

0.679
Aroclor-1016

Aroclor -1260

Aroclor -1260
Aroclor-1016 
HNF-SD-WM-DP-306 REV. 0

Data File: /chem/gc2.i/052198.b/009f0401.d Report Date: 28-May-1998 11:07

Page 2

RT

28.127

28.517

28.797

29.231

29.678

29.957

30.995

31.310

31.571

32.454

34.141

34.567

34.873

37.769

42.725

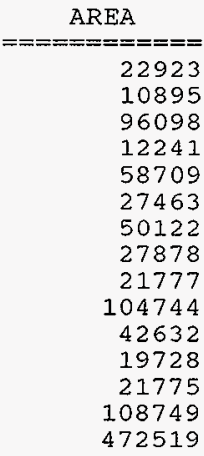

Total area $=3335398$
HT/AREA \% AREA

$======$

0.039

0.035

0.034

0.035

0.037

0.037

0.037

0.037

0.026

0.033

0.032

0.036

0.034

0.014

0.023 $\begin{aligned}== & ==== \\ & 0.687\end{aligned}$

0.327

2.88

0.367

1.76

0.823

1.50

0.836

0.653

3.14

1.28

0.591

0.653

3.26

14.2
Compounds

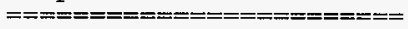

Aroclor -1260

Decachlorobiphenyl
744
1557

10872 


\section{2-S Laboratory}

Data file:/chem/gc2.i/052198.b/014f0401.d
Lab Smp Id: 100ng/ml A1248
Inj Date : 22-MAY-98 06:55
Client Smp ID: $100 \mathrm{ng} / \mathrm{ml}$ A1248
Operator : Gerald Ross
Smp Info : 100ng/ml A1248
Inst ID: $9 \mathrm{C} 2 . \mathrm{i}$
Misc Info : $100 \mathrm{ng} / \mathrm{ml}$ A1248
comment
Method
/chem/gc2.i/052198.b/rcrapcb.m
Meth Date : 28-May-1998 11:07
Cal Date : 22-MAY-98 06:55
Als bottle: 1
Dil Factor: 1.000
Integrator: HP Genie
Target Version: 3.10
Quant Type: AREA\%
Cal File: 014f0401.d
Calibration Sample, Level: 3
Compound sublist: AR1248. sub
Sample Matrix: WATER

\begin{tabular}{|c|c|}
\hline$=====$ & $\begin{array}{c}\mathrm{AREA} \\
========\end{array}$ \\
\hline 11.739 & 1242 \\
\hline 12.655 & 7691 \\
\hline 15.374 & 271 \\
\hline 16.157 & 156 \\
\hline 17.169 & 415 \\
\hline 17.245 & 3 \\
\hline 17.686 & 322 \\
\hline 18.028 & 1313 \\
\hline 18.271 & 1414 \\
\hline 18.538 & 1014 \\
\hline 18.688 & 677 \\
\hline 18.907 & 430 \\
\hline 19.105 & 31 \\
\hline 19.692 & 6 \\
\hline 19.876 & 265 \\
\hline 20.217 & 1165 \\
\hline 20.717 & 120 \\
\hline 21.362 & 330 \\
\hline 21.525 & 729 \\
\hline 21.740 & 815 \\
\hline 22.609 & 500 \\
\hline 22.855 & 380 \\
\hline 23.130 & 143 \\
\hline 23.999 & 12 \\
\hline 24.263 & 173 \\
\hline 24.785 & 318 \\
\hline 26.109 & 237 \\
\hline 27.585 & 14 \\
\hline 36.489 & 79 \\
\hline 42.728 & 376 \\
\hline
\end{tabular}

HEIGHT
$======$
729
44440
1308
579
1995
1702
1156
540
585
440
2776
1892
813
2664
888
3454
511
1329
2865
2675
1504
1281
597
469
643
1127
775
504
95
8800

HT/AREA \% AREA

0.059

0.058

0.048

0.037

0.048

0.045

0.036

0.041

0.041

0.043

0.041

0.044

0.025

0.042

0.033

0.030

0.042

0.040

0.039

0.033

0.030

0.034

0.041

0.038

0.037

0.035

0.033

0.034

0.001

0.023

$==$

0.570

35.3

1.24

0.718

1.91

1.73

1. 48

0.602

0.648

0.465

3.11

1.97

1. 47

2.88

1.22

5.34

0.553

1. 52

3.35

3.74

2.30

1.74

0.660

0.569

0.797

1.46

1.09

0.676

3.62

17.3
Compounds

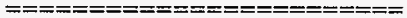

Tetrachloro-m-xylene

Aroclor -1248

Aroclor -1248

Aroclor -1248

Decachlorobiphenyl

Total area $=2180157$ 
Data File: /chem/gc2.i/052198.b/018f0401.d

HNF-SD-WM-DP-306 REV. 0

Report Date: 28-May-1998 11:08

222-S Laboratory

Data file: /chem/gc2.i/052198.b/018f0401.d

Lab Smp Id: $200 \mathrm{ng} / \mathrm{ml}$ A 1242

Inj Date : 22-MAY-98 10:38

Operator : Gerald Ross

Smp Info : $200 \mathrm{ng} / \mathrm{ml}$ A1242

Client Smp ID: $200 \mathrm{ng} / \mathrm{ml}$ A1242

Misc Info: $200 \mathrm{ng} / \mathrm{ml} \mathrm{A} 1242$

comment :

Method : /chem/gc2.i/052198.b/rcrapcb.m

Meth Date : 28-May-1998 11:08

Cal Date : 22-MAY-98 10:38

Als bottle: 1

Dil Factor: 1.000

Integrator: HP Genie

Target Version: 3.10

Inst ID: $g \mathrm{C} 2 . \mathrm{i}$

\begin{tabular}{|c|c|c|c|c|}
\hline $\begin{array}{c}\mathrm{RT} \\
====\end{array}$ & $\begin{array}{c}\mathrm{AREA} . \\
==========\end{array}$ & $==$ & $\begin{array}{l}\mathrm{HT} / \text { AREA } \\
======\end{array}$ & $\begin{array}{l}\% \text { AREA } \\
======\end{array}$ \\
\hline 10.630 & 10740 & 666 & 0.062 & 0.2 \\
\hline 11.739 & 10371 & 600 & 0.058 & 0.24 \\
\hline 12.545 & 11133 & 730 & 0.066 & 0.26 \\
\hline 12.655 & 1609175 & 89892 & 0.056 & \\
\hline 13.285 & 13752 & 579 & 0.042 & 0.3 \\
\hline 13.689 & 32847 & 1034 & 0.031 & \\
\hline 13.853 & 79054 & 3751 & 0.047 & 1.8 \\
\hline 14.610 & 11589 & 550 & 0.047 & 0.2 \\
\hline 15.372 & 102523 & 5007 & 0.049 & 2 . \\
\hline 15.470 & 62835 & 2524 & 0.040 & 1. \\
\hline 16.159 & 81697 & 3229 & 0.040 & 1. \\
\hline 16.807 & 22932 & 1067 & 0.047 & 0.5 \\
\hline 16.936 & 12014 & 533 & 0.044 & 0.2 \\
\hline 17.167 & 115209 & 5831 & 0.051 & \\
\hline 17.245 & 146268 & 6341 & 0.043 & \\
\hline 17.678 & 106136 & 4353 & 0.041 & \\
\hline 18.026 & 60338 & 2417 & 0.040 & 1. \\
\hline 18.271 & 20713 & 860 & 0.042 & 0.4 \\
\hline 18.688 & 78787 & 3128 & 0.040 & 1 . \\
\hline 18.908 & 55234 & 2437 & 0.044 & 1 . \\
\hline 19.106 & 42270 & 1107 & 0.026 & 0.9 \\
\hline 19.692 & 77809 & 3316 & 0.043 & 1. \\
\hline 19.888 & 59001 & 1779 & 0.030 & 1. \\
\hline 20.218 & 125070 & 3282 & 0.026 & 2 . \\
\hline 20.718 & 19818 & 747 & 0.038 & 0.4 \\
\hline 21.364 & 38375 & 1572 & 0.041 & 0.9 \\
\hline 21.526 & 81575 & 3214 & 0.039 & $1 \%$ \\
\hline 21.737 & 88198 & 3028 & 0.034 & 2 . \\
\hline 22.611 & 64666 & 1911 & 0.030 & 1. \\
\hline 22.856 & 28483 & 948 & 0.033 & 0.6 \\
\hline 24.265 & 12635 & 461 & 0.036 & 0 \\
\hline 24.789 & 5081 & 824 & 0.033 & \\
\hline
\end{tabular}

Compounds

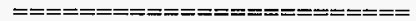

Tetrachloro-m-xylene

Aroclor -1242

Aroclor -1242

Aroclor -1242 
HNF-SD-WM-DP-306 REV. 0

Data File: /chem/gc2.i/052198.b/018f0401.d Report Date: 28-May-1998 11:08

Page 2

\begin{tabular}{|c|c|c|c|c|c|}
\hline $\begin{array}{l}\mathrm{RT} \\
====\end{array}$ & $\begin{array}{c}\mathrm{AREA} \\
=========\end{array}$ & $\begin{array}{c}\text { HETGHT } \\
==========\end{array}$ & $\begin{array}{l}\mathrm{HT} / \mathrm{AREA} \\
======\end{array}$ & $\begin{array}{l}\% \text { AREA } \\
======\end{array}$ & $\begin{array}{l}\text { Compounds } \\
=====================1\end{array}$ \\
\hline $\begin{array}{l}26.111 \\
36.496 \\
37.825 \\
42.727\end{array}$ & $\begin{array}{r}15567 \\
72033 \\
33319 \\
804458\end{array}$ & $\begin{array}{r}503 \\
189 \\
446 \\
18435\end{array}$ & $\begin{array}{l}0.032 \\
0.003 \\
0.013 \\
0.023\end{array}$ & $\begin{array}{r}0.368 \\
1.70 \\
0.787 \\
19.0\end{array}$ & Decachlorobiphenyl \\
\hline
\end{tabular}

Total area $=4231703$ 


$$
\text { 222-S Laboratory }
$$

Data file : /chem/gc2.i/052198.b/021f0401.d
Lab Smp Id: $200 \mathrm{ng} / \mathrm{ml}$ A1232
Inj Date : 22-MAY-98 13:35
Client Smp ID: 200ng/ml A1232
Operator : Gerald Ross
Smp Info : 200ng/ml A1232
Inst ID: $g c 2 . i$
Misc Info : $200 \mathrm{ng} / \mathrm{ml}$ A1232
comment :
Method : /chem/gc2.i/052198.b/rcrapcb.m
Meth Date : 28-May-1998 11:08
Cal Date : 22-MAY-98 13:35
Als bottle: 1
Dil Factor: 1.000
Integrator: HP Genie
Target Version: 3.10
Quant Type: AREA\%
Cal File: 021f0401.d
Calibration Sample, Level: 4

\begin{tabular}{|c|c|c|c|c|}
\hline$==\stackrel{\mathrm{RT}}{==}=$ & $\begin{array}{c}\mathrm{AREA} \\
==== \pm=====\end{array}$ & $\begin{array}{c}\text { HEIGHT } \\
=========\end{array}$ & $\begin{array}{l}\mathrm{HT} / \mathrm{AREA} \\
======\end{array}$ & $\begin{array}{l}\% \text { AREA } \\
======\end{array}$ \\
\hline 10.631 & 10305 & 692 & 0.067 & 0.26 \\
\hline 10.818 & 31625 & 1809 & 0.057 & 0.82 \\
\hline 11.741 & 12045 & 629 & 0.052 & 0.3 \\
\hline 11.916 & 11997 & 580 & 0.048 & 0.3 \\
\hline 12.545 & 18901 & 1162 & 0.061 & 0.4 \\
\hline 12.656 & 1609727 & 90311 & 0.056 & 41. \\
\hline 13.291 & 37071 & 1608 & 0.043 & 0.96 \\
\hline 13.648 & 44925 & 1530 & 0.034 & \\
\hline 13.855 & 111848 & 5155 & 0.046 & \\
\hline 15.375 & 70166 & 3038 & 0.043 & \\
\hline 15.481 & 52872 & 1796 & 0.034 & \\
\hline 16.163 & 48394 & 1893 & 0.039 & \\
\hline 16.810 & 14035 & 647 & 0.046 & 0.36 \\
\hline 17.171 & 67280 & 3434 & 0.051 & \\
\hline 17.248 & 87570 & 3791 & 0.043 & 2. \\
\hline 17.681 & 67441 & 2624 & 0.039 & 1. \\
\hline 18.030 & 35113 & 1394 & 0.040 & 0.91 \\
\hline 18.273 & 12763 & 512 & 0.040 & 0.3 \\
\hline 18.692 & 38754 & 1556 & 0.040 & 1. \\
\hline 18.911 & 28591 & 1273 & 0.045 & 0.7 \\
\hline 19.116 & 23794 & 631 & 0.027 & 0.6 \\
\hline 19.696 & 38674 & 1646 & 0.043 & 1. \\
\hline 19.894 & 33287 & 1014 & 0.030 & 0.8 \\
\hline 20.219 & 130312 & 4661 & 0.036 & 3. \\
\hline 21.368 & 21113 & 857 & 0.041 & 0.5 \\
\hline 21.531 & 42750 & 1674 & 0.039 & 1. \\
\hline 21.741 & 46847 & 1607 & 0.034 & 1. \\
\hline 22.617 & 35157 & 1023 & $0.029^{\circ}$ & 0.91 \\
\hline 22.860 & 13213 & 435 & 0.033 & 0.34 \\
\hline 24.795 & 12255 & 377 & 0.031 & 0.3 \\
\hline 28.103 & 11047 & 380 & 0.034 & 0.2 \\
\hline 36.499 & 78639 & 231 & 0.003 & 2 \\
\hline
\end{tabular}

Compounds

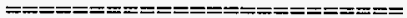

Tetrachloro-m-xylene

Aroclor -1232

Aroclor -1232

Aroclor -1232 


\section{HNF-SD-WNA-DP-306 REV. 0}

Data File: /chem/gc2.i/052198.b/021f0401.d

Page 2

Report Date: 28-MaY-1998 11:08

\begin{tabular}{|c|c|c|c|c|c|}
\hline $\begin{array}{l}\mathrm{RT} \\
=====\end{array}$ & $\begin{array}{c}\mathrm{AREA} \\
=========\end{array}$ & $\begin{array}{c}\text { HEIGHT } \\
==========\end{array}$ & $\begin{array}{l}\mathrm{HT} / \mathrm{AREA} \\
=======\end{array}$ & $\begin{array}{l}\% \text { AREA } \\
======\end{array}$ & $\begin{array}{l}\text { Compounds } \\
\text { Com }\end{array}$ \\
\hline $\begin{array}{l}37.843 \\
42.735\end{array}$ & $\begin{array}{r}43576 \\
914759\end{array}$ & $\begin{array}{r}585 \\
21037\end{array}$ & $\begin{array}{l}0.013 \\
0.023\end{array}$ & $\begin{array}{l}1.13 \\
23.7\end{array}$ & Decachlorobiphenyl \\
\hline
\end{tabular}

Total area $=3856845$ 


\section{2-S Laboratory}

Data file:/chem/gc2.i/052198.b/024f0401.d

Lab Smp Id: 400ng/mlA1221

Inj Date : 22-MAY-98 16:26

Operator : Gerald Ross

Smp Info : 400ng/mlA1221

Misc Info: $400 \mathrm{ng} / \mathrm{mlA} 1221$

comment :

Method : /chem/gc2.j/052198.b/rcrapcb.m

Meth Date : 28-May-1998 11:08

Cal Date : 22-MAY-98 16:26

Als bottle: 1

Dil Factor: 1.000

Integrator: HP Genie

Target Version: 3.10

\begin{tabular}{|c|c|c|c|c|}
\hline$==\begin{array}{c}\mathrm{RT} \\
===\end{array}$ & $\begin{array}{c}\text { AREA } \\
==========\end{array}$ & $\begin{array}{c}\text { HEIGHT } \\
=========\end{array}$ & $\begin{array}{l}\mathrm{HT} / \mathrm{AREA} \\
======\end{array}$ & $\begin{array}{l}\% \text { AREA } \\
=======\end{array}$ \\
\hline 10.814 & 81132 & 4680 & 0.058 & 1.67 \\
\hline 740 & 12793 & 616 & 0.048 & 0.263 \\
\hline 11.911 & 34199 & 1653 & 0.048 & 0.704 \\
\hline 12.543 & 37851 & 2314 & 0.061 & 0.779 \\
\hline 12.655 & 2559259 & 140271 & 0.055 & 52.6 \\
\hline 3.290 & 89293 & 3895 & 0.044 & 1.84 \\
\hline 3.644 & 90833 & 3276 & 0. & 1.87 \\
\hline 3.851 & 212762 & 9454 & 0. & 4.38 \\
\hline 5.292 & 21122 & 965 & 0 . & 0.43 \\
\hline 5.371 & 13200 & 664 & 0. & 0.27 \\
\hline 15.507 & 50178 & 1870 & 0. & 1.0 \\
\hline 16.595 & 10078 & 408 & 0. & 0.20 \\
\hline 17.250 & 15248 & 638 & 0.042 & 0.31 \\
\hline 17.683 & 13679 & 480 & 0.035 & 0.28 \\
\hline 20.218 & 25294 & 1073 & 0.042 & 0.52 \\
\hline 36.497 & 79376 & 344 & 0.004 & 1.6 \\
\hline 37.834 & 60575 & 830 & 0.014 & 1.2 \\
\hline & 14116 & 379 & & 0.29 \\
\hline 2.732 & 1439966 & 32375 & 0.022 & 29. \\
\hline
\end{tabular}

Quant Type: AREA\%

Cal File: 024f0401.d

Calibration Sample, Level: 4

Compound Sublist: AR1221.sub

Sample Matrix: WATER

Compounds

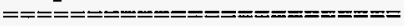

Aroclor -1221

Tetrachloro-m-xylene

Aroclor-1221

Aroclor -1221

Decachlorobiphenyl

Total area $=4860956$ 
Data File: /chem/gc2.i/052198.b/029f0401.d

Report Date: 29-May-1998 11:32

222-S Laboratory

Data file: /chem/gc2.i/052198.b/029f0401.d
Lab Smp Id: 992 LCS/100
Inj Date : 22-MAY-1998 21:09
operator : Gerald Ross
Smp Info : $992 \mathrm{LCS} / 100$
Client Smp ID: 992 LCS/100
Misc Info : $992 \mathrm{LCS} / 100$
Inst ID: $g C 2 . i$
comment :
Method : /chem/gc2.i/052198.b/rcrapcb.m
Meth Date : 29-May-1998 10:43 gar
Cal Date : 22-MAY-1998 20:13
Als bottle: 1
Dil Factor: 100.000
Integrator: HP Genie
Target Version: 3.10
Quant Type: AREA\%
Cal File: 028f0401.d
QC Sample: METHSPIKE
Compound Sublist: AR1254, sub
Sample Matrix: SOIL

\begin{tabular}{|c|c|c|c|c|c|}
\hline$== \pm===$ & $\begin{array}{c}\mathrm{AREA} \\
===========\end{array}$ & $\begin{array}{c}\text { HEIGHT } \\
===========\end{array}$ & $\begin{array}{l}\text { HT / AREA } \\
=======\end{array}$ & $\begin{array}{l}\% \text { AREA } \\
=======\end{array}$ & $\begin{array}{l}\text { Compounds } \\
==================m=1\end{array}$ \\
\hline 11.729 & 15522 & 834 & 0.054 & 0.422 & \\
\hline 11.784 & 20565 & 927 & 0.045 & 0.559 & \\
\hline 12.655 & 935147 & 52932 & 0.057 & 25.4 & Tetrachloro-m-xylene \\
\hline 15.874 & 17441 & 875 & 0.050 & 0.474 & \\
\hline 18.688 & 101776 & 4403 & 0.043 & 2.77 & \\
\hline 18.907 & 24062 & 1015 & 0.042 & 0.654 & \\
\hline 19.452 & 45273 & 1736 & 0.038 & 1.23 & \\
\hline 19.691 & 49632 & 2107 & 0.042 & 1.35 & \\
\hline 20.217 & 40864 & 1243 & 0.030 & 1.11 & \\
\hline 21.362 & 17070 & 686 & 0.040 & 0.464 & \\
\hline 21.525 & 75717 & 3025 & 0.040 & 2.06 & \\
\hline 2.1 .765 & 170165 & 6371 & 0.037 & 4.63 & Aroclor-1254 \\
\hline 22.097 & 22813 & 934 & 0.041 & 0.620 & \\
\hline 22.556 & 46429 & 1594 & 0.034 & 1.26 & \\
\hline 22.844 & 226271 & 8030 & 0.035 & 6.15 & Aroclor -1254 \\
\hline 23.128 & 65824 & 2690 & 0.041 & 1.79 & \\
\hline 23.727 & 10583 & 424 & 0.040 & 0.288 & \\
\hline 23.996 & 57213 & 2244 & 0.039 & 1.56 & \\
\hline 24.259 & 105281 & 4006 & 0.038 & 2.86 & \\
\hline 24.507 & 28474 & 1130 & 0.040 & 0.774 & \\
\hline 24.780 & 209082 & 7388 & 0.035 & 5.68 & Aroclor -1254 \\
\hline 25.410 & 41777 & 1397 & 0.033 & 1.14 & \\
\hline 25.626 & 29740 & 1065 & 0.036 & 0.808 & \\
\hline 25.837 & 13425 & 518 & 0.039 & 0.365 & \\
\hline 26.101 & 228634 & 5448 & 0.024 & 6.22 & \\
\hline 26.638 & 12179 & 404 & 0.033 & 0.331 & \\
\hline $\begin{array}{l}26.924 \\
27.251\end{array}$ & 21211 & 672 & 0.032 & 0.577 & \\
\hline 27.560 & $\begin{array}{r}86842 \\
112919\end{array}$ & $\begin{array}{l}3270 \\
2903\end{array}$ & $\begin{array}{l}0.038 \\
0.026\end{array}$ & $\begin{array}{l}2.36 \\
3.07\end{array}$ & \\
\hline 27.973 & 18315 & 799 & 0.044 & 0.498 & \\
\hline 28.369 & 10966 & 405 & 0.037 & 0.298 & \\
\hline 28.510 & 13946 & 480 & 0.034 & 0.379 & \\
\hline
\end{tabular}




\section{HNF-SD-WM-DP-306 REV. 0}

Data File: /chem/gc2.i/052198.b/029f0401.d

Report Date: 29-May-1998 11:32

Page 2

RT

$==ニ==$
28.801

28.949

29.275

30.355

31.595

32.459

36.492

42.726
AREA

$$
145923
$$

19407

10871

38135

18123

16902

47499

506215
HEIGHT

$========$

HT/AREA \% AREA

$====$

0.038

0.034

0.031

0.034

0.029

0.002

0.023
$=====$
3.97

0.528

0.296

1.04

0.493

0.460

1.29

13.8
Compounds

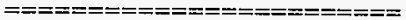

Decachlorobiphenyl

Total area $=3678230$

$-935,147$

$-506215$

$2,236,868$ $\frac{2,236,868}{4,200,793}$
LCS Recovery

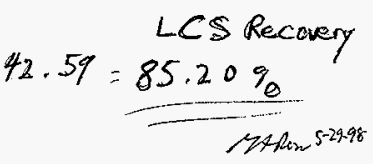


Data File: /chem/gc2.i/052198.b/029f0401.d

Report Date: 29-May-1998 11:32

Data file: /chem/gc2.i/052198.b/029f0401.d

Lab Smp Id: 992 LCS/100

Inj Date : 22-MAY-1998 21:09

operator : Gerald Ross

Client Smp ID: 992 LCS $/ 100$

Smp Info: $992 \mathrm{LCS} / 100$

Inst ID: $g C 2 . i$

Misc Info : 992 LCS $/ 100$

Comment :

Method : /chem/gc2.i/052198.b/rcrapcb.m

Meth Date : 29-May-1998 10:43 gar Quant Type: ESTD

Cal Date : 22-MAY-1998 20:13

Als bottle: 1

Dil Factor: 100.000

Integrator: HP Genie

Target Version: 3.10

Cal File: 028f0401,d

QC Sample: METHSPIKE

Compound Sublist: AR1254.sub

Sample Matrix: SoIL

\begin{tabular}{|c|c|c|c|c|c|c|c|}
\hline \multirow[b]{3}{*}{$\mathrm{RI}$} & \multirow{3}{*}{\multicolumn{2}{|c|}{ EXP RT DLT RT }} & & \multirow[b]{3}{*}{ TARGET RANGE } & \multirow[b]{3}{*}{ RATIO } \\
\hline & & & & ON-COL & FINAL & & \\
\hline & & & RESPONSE & ( $\mathrm{ng})$ & $(u g / K g)$ & & \\
\hline$\Rightarrow$ & $===x==$ & =E=ニ=ニ=== & $=======$ & $= \pm= \pm===$ & $=\overline{=}=\overline{=}==$ & $= \pm= \pm== \pm=== \pm==$ & $====$ \\
\hline \multicolumn{3}{|c|}{27 Arocior -1254} & & \multicolumn{3}{|c|}{ CAS \#: $11097-69-1$} & \\
\hline $21: 765$ & 21.767 & $7-0.002$ & 170164 & 0.209 & 41700 & & 100.00 \\
\hline 22.844 & 22.846 & $5-0.002$ & 226271 & 0.210 & 42000 & $112.00-152.00$ & 132.97 \\
\hline 24.780 & 24.780 & 0.000 & 209082 & 0.216 & 43200 & $96.00-136.00$ & 122.87 \\
\hline \multicolumn{3}{|c|}{ Average of Pea } & \multicolumn{3}{|r|}{42300} & & \\
\hline \multicolumn{4}{|c|}{$\$ 29$ Ietrachloro-m-xylene } & \multicolumn{4}{|c|}{ CAS \#: $877-09-8$} \\
\hline 12.655 & 12.654 & 0.001 & 935147 & 0.0634 & 12700 & & 100.00 \\
\hline \multicolumn{4}{|c|}{ \$ 30 Decachlorobiphenyl } & \multicolumn{4}{|c|}{ CAS \#: $2051-24-3$} \\
\hline 42.726 & 42.728 & $8-0.002$ & 506214 & 0.0622 & 12400 & & 100.00 \\
\hline
\end{tabular}


Data File: /chem/gc2.i/052198.b/029f0401.d

Report Date: 29-May-1998 13:24

Page 1

\section{2-S Laboratory}

Data file: /chem/gc2.i/052198.b/029f0401.d
Lab Smp. Id: 992 LCS $/ 100$
Inj Date : 22-MAY-1998 21:09
operator : Gerald Ross
Client Smp ID: 992 LCS/100
Smp Info : $992 \mathrm{LCS} / 100$
Inst ID: $g c 2, i$
Misc Info : $992 \mathrm{LCS} / 100$
comment :
Method : /chem/gc2.i/052198.b/rcrapcb.m
Meth Date : 29-May-1998 13:08 gar Quant Type: ESTD
Cal Date : 22-MAY-1998 20:13 Cal File: 028f0401.d
Als bottle: 1
Dil Factor: 100.000
Integrator: HP Genie
Target Version: 3.10 QC Sample: METHSPIKE
Compound Sublist: AR1254.sub Sample Matrix: SOIL

\begin{tabular}{|c|c|c|c|c|c|c|c|}
\hline & & & & CONCENTR & ATIONS & & \\
\hline & & & & $\mathrm{ON}-\mathrm{COL}$ & FINAL & & \\
\hline RT & EXP RT & DLI RT & RESPONSE & ( $n g)$ & $(u g / K g)$ & TARGET RANGE & RATIO \\
\hline$==$ & =ニニニニ== & $=======-$. & $==\pi= \pm===$ & $== \pm====$ & $====-==$ & $============$ & $==E==$ \\
\hline $27 \mathrm{Ar}$ & roctor -12 & 254 & & & CAS \#: & $11097-69-1$ & \\
\hline 21.765 & 21.767 & -0.002 & 170164 & 0.209 & 41700 & & 100.00 \\
\hline 22.844 & 22.846 & -0.002 & 226271 & 0.210 & 42000 & $112.00-152.00$ & 132.97 \\
\hline 24.780 & 24.780 & 0.000 & 209082 & 0.216 & 43200 & $96.00-136.00$ & 122.87 \\
\hline & & Average 0 & Concentra & ations = & 42300 & & \\
\hline$\$ 29 \mathrm{Te}$ & etrachlor & ro-m-xyle & & & CAS \#: & & \\
\hline 12.655 & 12.654 & 0.001 & 935147 & 0.0634 & 12700 & & 100.00 \\
\hline$\$ 300$ & ecachlor & obiphenyl & - & & CAS \#: & $2051-24-3$ & \\
\hline 42.726 & 42.728 & .0 .002 & 506214 & 0.0622 & $12400^{\circ}$ & & 100.00 \\
\hline
\end{tabular}


Data File: /chem/gc2.i/052198.b/029f0401.d

$$
\text { 222-S Laboratory }
$$

\section{RECOVERY REPORT}

Client Name:

Sample Matrix: SOLID

Lab Simp Id: $992 \mathrm{LCS} / 100$

Level: LOW

Data Type: GC MULTI COMP

Spikelist File: Aroclor-1254.spk

Method File: /chem/gc2.i/052198.b/rcrapcb.m

Client SDG: 052198

Fraction: PEST

Client Smp ID: 992 LCS/100

Operator: Gerald Ross

SampleType: METHSPIKE

Quant Type: ESTD

Misc Info: $992 \mathrm{LCS} / 100$

\begin{tabular}{|c|c|c|c|c|}
\hline SPIKE COMPOUND & $\begin{array}{l}\text { CONC } \\
\text { ADDED } \\
u g / K g\end{array}$ & $\begin{array}{c}\text { CONC } \\
\text { RECOVERED } \\
\text { ug/Kg }\end{array}$ & RECOVERED & LIMITS \\
\hline 27 Aroclor -1254 & 50000 & $\begin{array}{l}42300 \\
42,590\end{array}$ & $\begin{array}{ll}\left(3 P_{K}\right) & 84.62 \\
\text { (TorAMAK) } & 85,20 \\
\end{array}$ & $\overline{50-150}$ \\
\hline SURROGATE COMPOUND & $\begin{array}{l}\text { CONC } \\
\text { ADDED } \\
\mathrm{ug} / \mathrm{Kg}\end{array}$ & $\begin{array}{l}\text { CONC } \\
\text { RECOVERED } \\
\text { ug/Kg }\end{array}$ & RECOVERED & LIMITS \\
\hline $\begin{array}{lll}\$ & 29 & \text { Tetrachloro-m-xyle } \\
\$ & 30 & \text { Decachlorobiphenyl }\end{array}$ & $\begin{array}{l}16000 \\
16000\end{array}$ & $\begin{array}{l}12700 \\
12400\end{array}$ & $\begin{array}{l}79.27 \\
77.78\end{array}$ & $\begin{array}{l}50-150 \\
50-150\end{array}$ \\
\hline
\end{tabular}


Data File: /chem/gc2.i/052198.b/029f0401.d

222-S Laboratory

TARGET COMPOUNDS

Client Name:

Lab Smp Id: 992 LCS/100

Sample Location:

Sample Date:

Sample Matrix: SoIL

Analysis Type: PEST

Data Type: GC MULTI COMP

Misc Info: 992 LCS $/ 100$
Client SDG: 052198

Client Smp ID: 992 LCS/100

Sample Point:

Date Received:

Quant Type: ESTD

Level: LOW

Operator: Gerald Ross

CAS NO.

COMPOUND

CONCENTRATION UNITS:

(ug/L or $u g / K G$ ) ug/Kg

Q

11097-69-1------Aroclor -1254

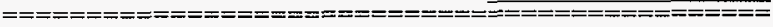

877-09-8------Tetrachloro-m-xylene

2051-24-3------Decachlorobiphenyl

$=====+\begin{array}{r}42300 \\ 12700 \\ 12400\end{array} \mid=====$




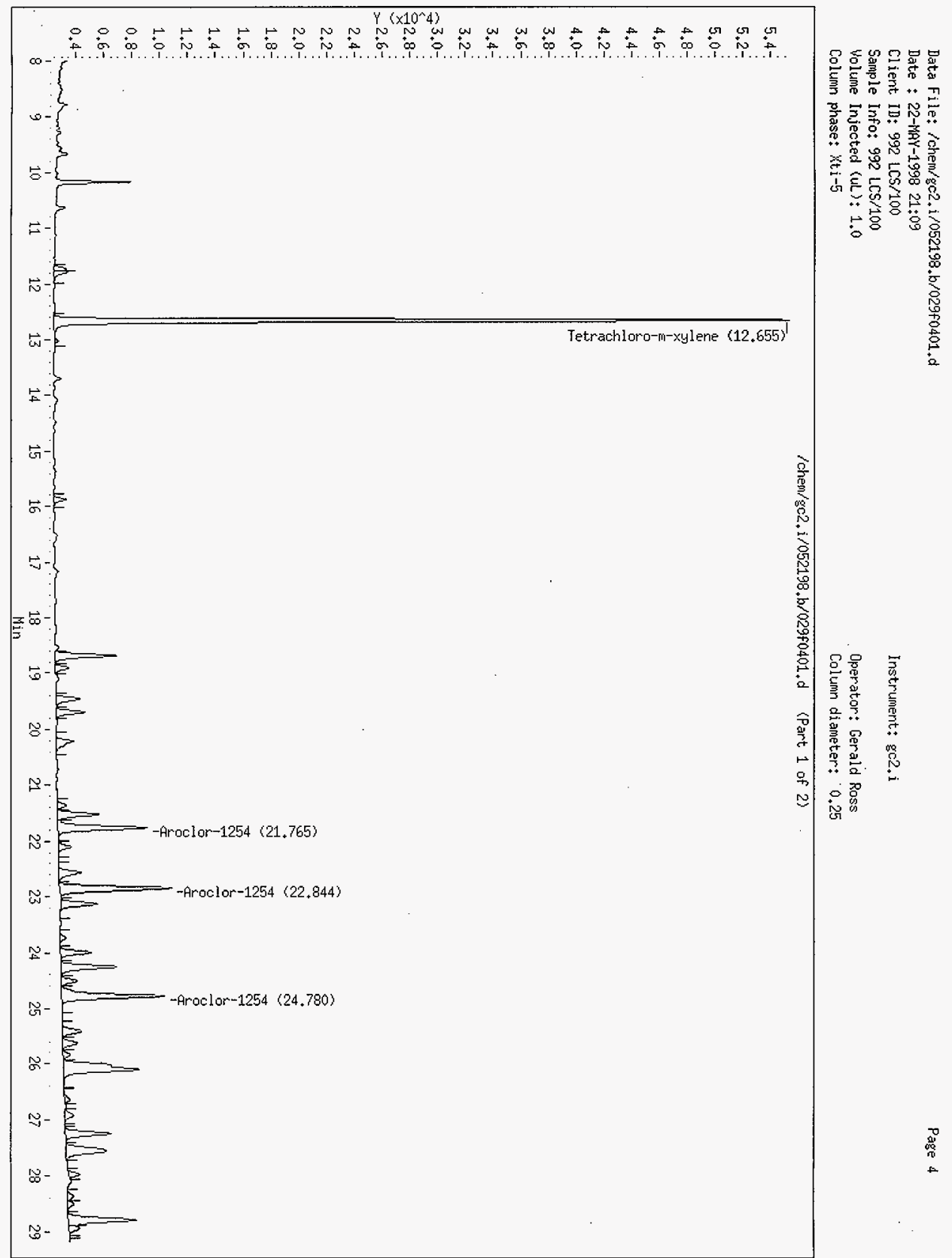


Data File: /chem/gc2, i/052198, b/029f0401,d

Date : 22-MAY-1998 21:09

Client IDะ $992 \mathrm{LCS} / 100$

Sample Info: $992 \mathrm{LCS} / 100$

Volume Injected $(u L): 1.0$

Column phase: $x_{t}-5$

Instrument: gc2.i

Dperator: Gerald Ross

Column diameter. 0.25

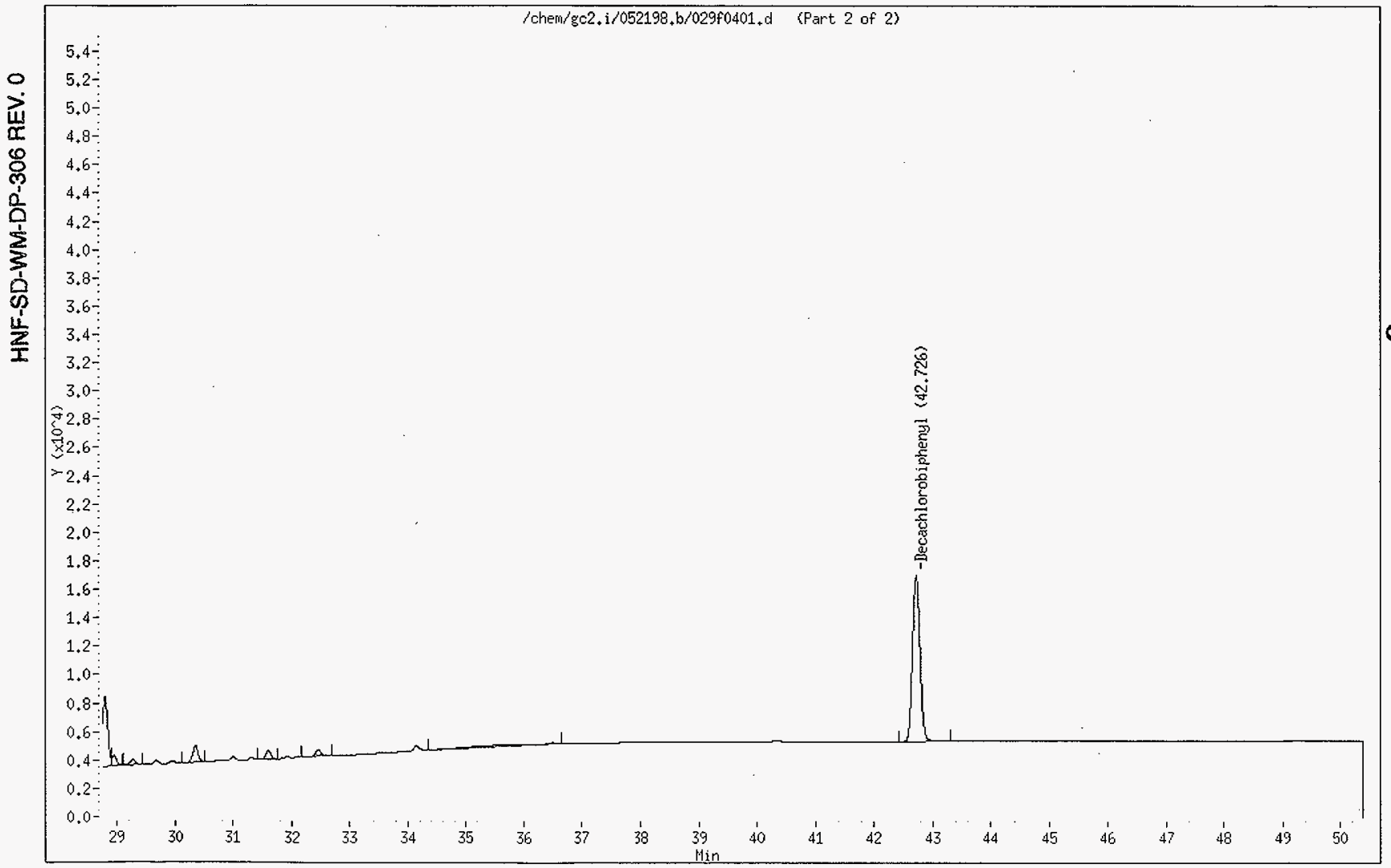


Data File: /chem/gc2.i/052198.b/030f0401.d

Page 1

Report Date: 29-May-1998 13:24

\section{2-S Laboratory}

Data file : /chem/gc2.i/052198.b/030f0401.d
Lab Smp Id: $990 \mathrm{Mblnk} / 100$
Inj Date : 22-MAY-1998 22:06
operator : Gerald Ross
Client Smp ID: $990 \mathrm{Mblnk} / 100$
Smp Info : $990 \mathrm{Mblnk} / 100$
Inst ID: gc2.i
Misc Info : $990 \mathrm{Mblnk} / 100$
comment
Method
/chem/gc2.i/052198.b/rcrapcb.m
Meth Date : 29-May-1998 13:08 gar
Cal Date: 22-MAY-1998 20:13
Quant Type: ESTD
Als bottle: 1
Dil Factor: 100.000
Integrator: HP Genie
Target Version: 3.10
Cal File: 028f0401.d
Compound Sublist: Aroclors.sub
Sample Matrix: SoIL

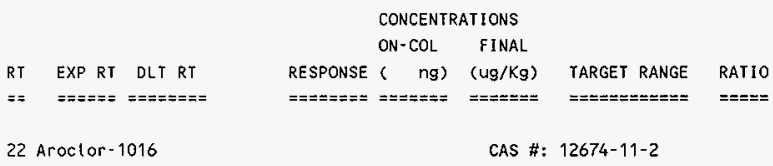

Peaks not detected for Quant. or Qual. signal(s).

23 Aroclor -1221

CAS \#: $11104-28-2$

Peaks not detected for Quant, or Qual. signal(s).

24 Aroctor -1232

CAS $\#: 1114-16-5$

Peaks not detected for Quant. or Quat. signal(s).

25 Aroctor -1242

CAS \#: $53469-21-9$

Peaks not detected for Quant. Or Qual. signal(s).

26 Aroclor -1248

CAS \#: $12672-29-6$

Peaks not detected for Quant. or Qual. signal(s).

27 Aroclor -1254

CAS \#: $11097-69-1$

Peaks not derected for Quant. or Qual. signal(s). 
Data File: /chem/gc2.i/052198.b/030f0401.d Report Date: 29-May-1998 13:24

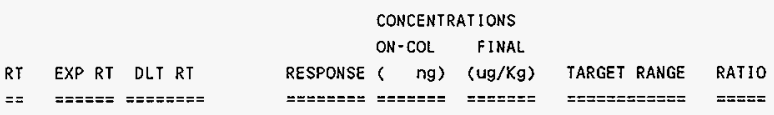

28 Aroclor -1260

CAS \#: $11096-82-5$

Peaks not detected for Quant. or Qual. signal(s).

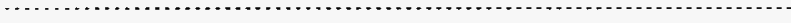

$\$ 29$ Tetrachloro-m-xylene CAS \#: $877-09-8$

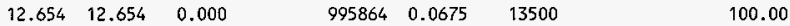

$\$ 30$ Decachtorobiphenyl

CAS \#: 2051-24-3

$\begin{array}{llll}42.722 & 42.728 & -0.006\end{array}$

$577760 \quad 0.0710$

14200

100.00 
Data File: /chem/gc2.i/052198.b/030f0401.d

Report Date: 29-May-1998 13:24

\section{2-s Laboratory}

\section{RECOVERY REPORT}

client Name:

Sample Matrix: SOLID

Lab Smp Id: $990 \mathrm{Mblnk} / 100$

Level: LOW

Data Type: GC MULTI COMP

SpikeList File: RESOLUTION.spk

Method File:/chem/gc2.i/052198.b/rcrapcb.m

Misc Info: $990 \mathrm{Mblnk} / 100$
Client SDG: 052198

Fraction: PEST

Client Smp ID: $990 \mathrm{Mblnk} / 100$

Operator: Gerald Ross

SampleType: SAMPLE

Quant Type: ESTD

\begin{tabular}{|c|c|c|c|c|}
\hline SURROGATE COMPOUND & $\begin{array}{l}\text { CONC } \\
\text { ADDED } \\
\mathrm{ug} / \mathrm{Kg}\end{array}$ & $\begin{array}{c}\text { CONC } \\
\text { RECOVERED } \\
\mathrm{ug} / \mathrm{Kg}\end{array}$ & RECOVERED & LIMITS \\
\hline $\begin{array}{lll}\$ & 29 & \text { Tetrachloro-m-xyle } \\
\$ & 30 & \text { Decachlorobiphenyl }\end{array}$ & $\begin{array}{l}16000 \\
16000\end{array}$ & $\begin{array}{l}13500 \\
14200\end{array}$ & $\begin{array}{l}84.42 \\
88.77\end{array}$ & $\begin{array}{l}50-150 \\
50-150\end{array}$ \\
\hline
\end{tabular}


Data File: /chem/gc2.i/052198.b/030f0401.d

Report Date: 29-May-1998 13:24

$$
\begin{aligned}
& \text { 222-S Laboratory } \\
& \text { TARGET COMPOUNDS }
\end{aligned}
$$

Client Name:

Lab Smp Id: $990 \mathrm{Mblnk} / 100$

Sample Location:

Sample Date:

Sample Matrix: SoIL

Analysis Type: PEST

Data Type: GC MULTI COMP

Misc Info: $990 \mathrm{Mblnk} / 100$
Client SDG: 052198

Client Smp ID: $990 \mathrm{Mblnk} / 100$

Sample Point:

Date Received:

Quant Type: ESTD

Level: LOW

Operator: Gerald Ross

CAS NO.

COMPOUND

CONCENTRATION UNITS:

(ug/L or $u g / K G$ ) ug/Kg

\begin{tabular}{|c|}
\hline $\begin{array}{l}877-09-8-\ldots-1 \\
2051-24-3-\cdots-\infty-0 \text { - Decachlorobiphenyl }\end{array}$ \\
\hline
\end{tabular}

Q

\begin{tabular}{r|r|}
3300 & $\mathrm{U}$ \\
6600 & $\mathrm{U}$ \\
3300 & $\mathrm{U}$ \\
3300 & $\mathrm{U}$ \\
3300 & $\mathrm{U}$ \\
3300 & $\mathrm{U}$ \\
3300 & $\mathrm{U}$ \\
13500 & \\
14200 & \\
\hline
\end{tabular}




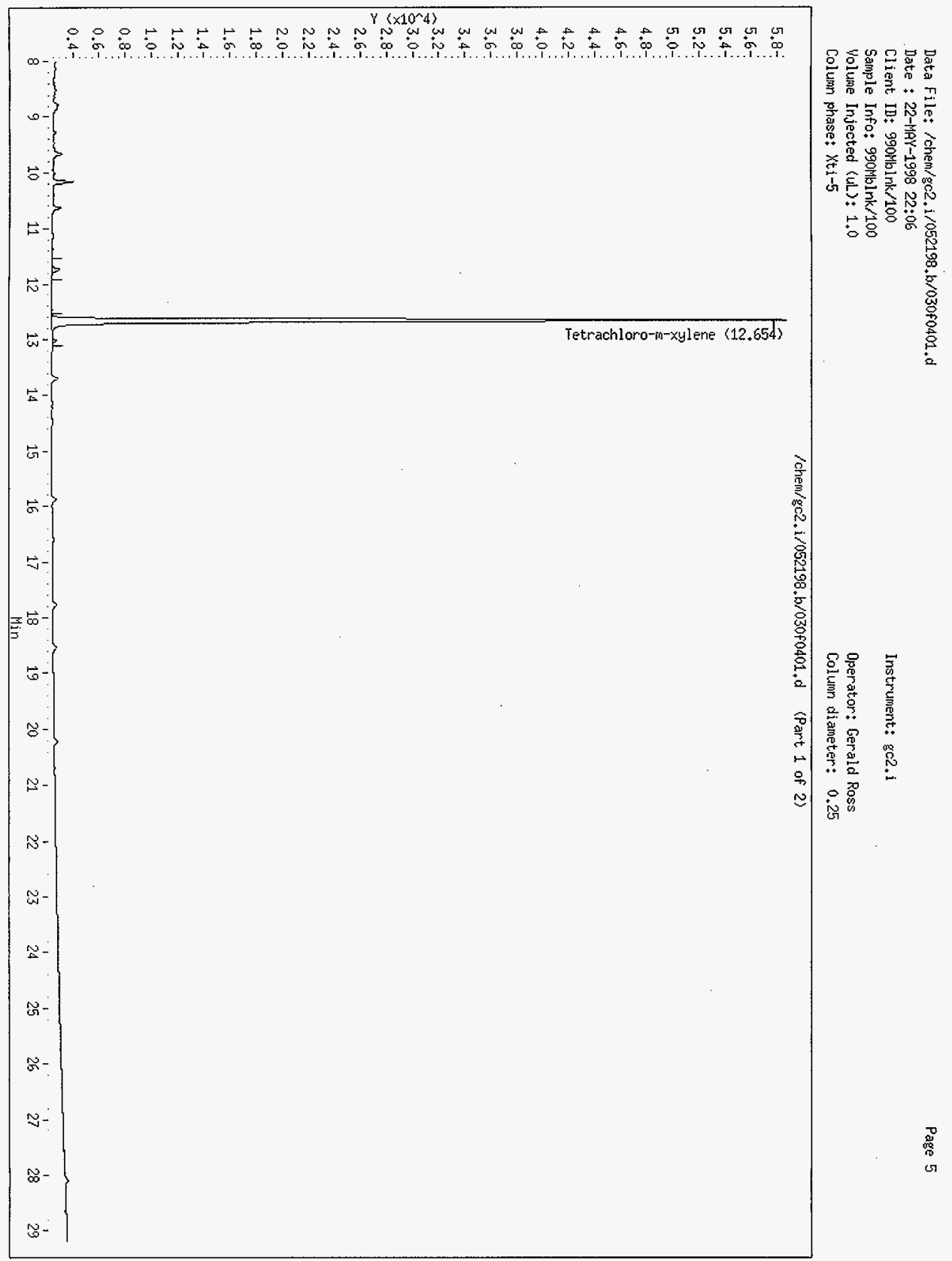


Data File: ichem/ge2, i/052198,b/030f0401,d

Date : 22-MAY-1998 22:06

Client ID: $990 \mathrm{Mblnk} / 100$

Sample Info: 990itbl nk/100

Volume Injected (uL): 1.0

Instrument: gc2.i

Column phase: Xti-5

Operator: Gerald Ross

Column diameter: 0,25

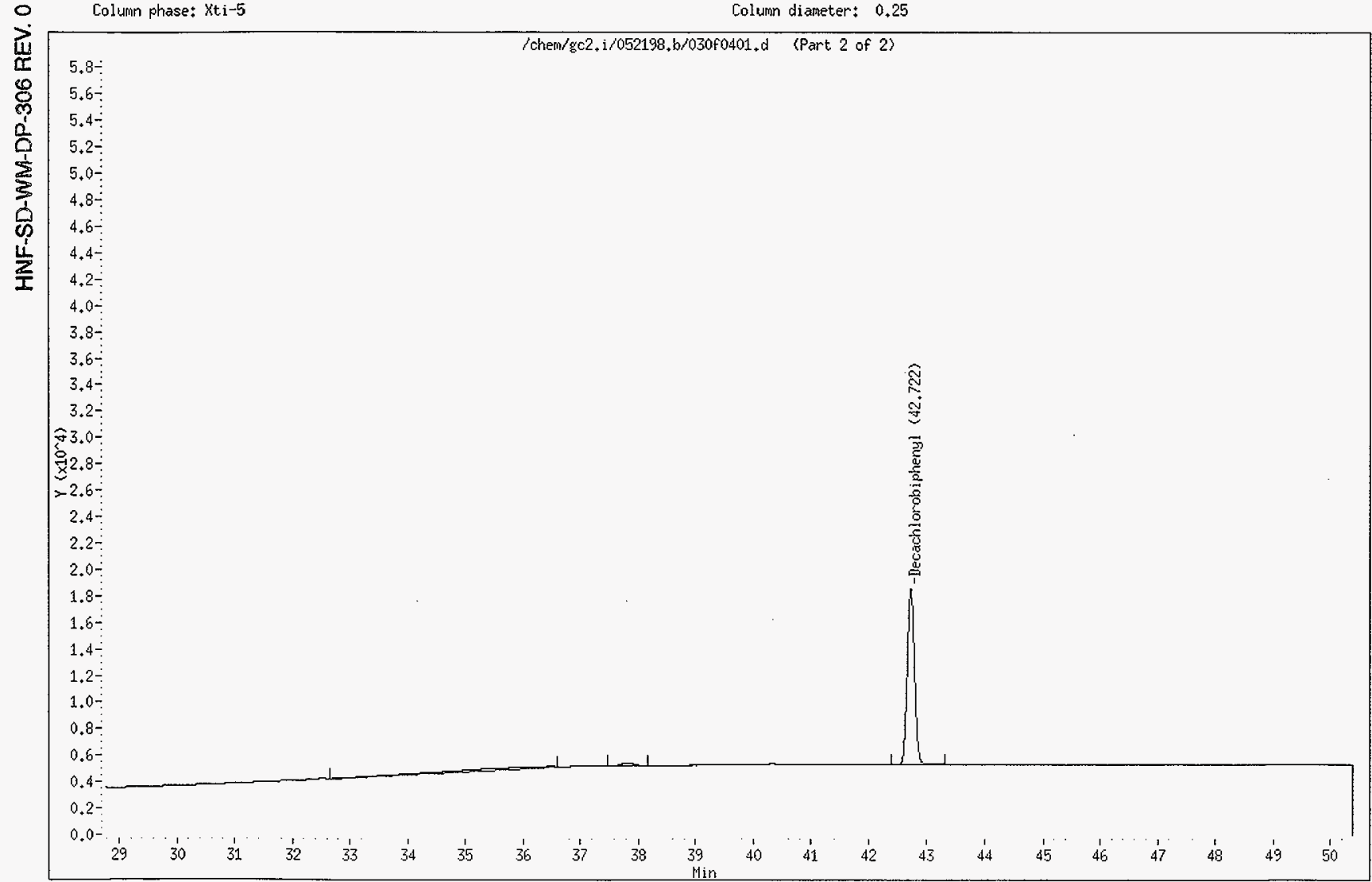




$$
\text { 222-s Laboratory. }
$$

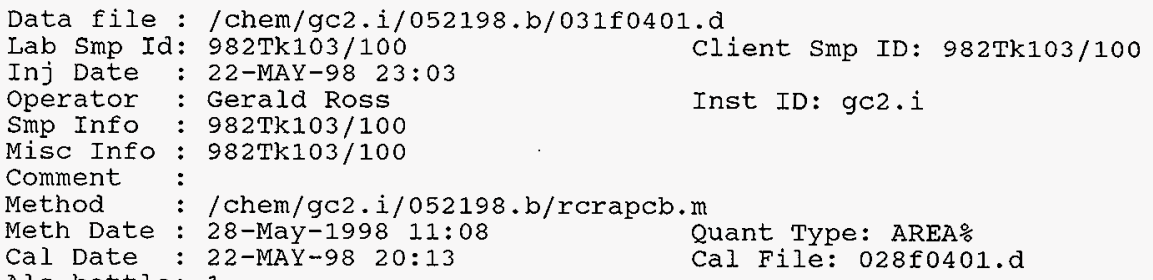

Als bottle: 1

Dil Factor: 100.000 Integrator: HP Genie Target Version: 3.10

Compound Sublist: Aroclors.sub Sample Matrix: SOIL

\begin{tabular}{|c|c|c|c|c|c|}
\hline$=\begin{array}{c}\mathrm{RT} \\
===\end{array}$ & $\begin{array}{c}\mathrm{AREA} \\
============\end{array}$ & $\begin{array}{l}\mathrm{HEIGHT} \\
========\end{array}$ & $\begin{array}{l}\mathrm{HT} / \mathrm{AREA} \\
======\end{array}$ & $\begin{array}{l}\% \text { AREA } \\
======\end{array}$ & $\begin{array}{l}\text { Compounds } \\
==============+=====1\end{array}$ \\
\hline 11.738 & 18030 & 941 & 0.052 & 0.402 & \\
\hline 11.907 & 21944 & 524 & 0.024 & 0.489 & \\
\hline 12.652 & $1021596(-)$ & 58471 & 0.057 & 22.8 & Tetrachloro-m-xylene \\
\hline 12.967 & $14114=$ & 557 & 0.039 & 0.314 & \\
\hline 13.307 & $43,21, ? 2$ & 966 & 0.035 & 0.616 & \\
\hline 13.558 & 20005 & 766 & 0.038 & 0.446 & \\
\hline 13.693 & 11680 & 594 & 0.051 & 0.260 & \\
\hline 14.386 & 45777 & 1162 & 0.025 & 1.02 & \\
\hline 14.712 & 18914 & 786 & 0.042 & 0.421 & \\
\hline 14.768 & $14648(-)$ & 719 & 0.049 & 0.326 & \\
\hline 15.158 & $21428(-)$ & 846 & 0.039 & 0.477 & \\
\hline 15.351 & 30461 & 603 & 0.020 & 0.679 & \\
\hline 16.168 & 17893 & 813 & 0.045 & 0.399 & \\
\hline 16.614 & 10007 & 434 & 0.043 & 0.223 & \\
\hline 16.799 & 28400 & 835 & 0.029 & 0.633 & \\
\hline 17.336 & $88792 !-$ & 2248 & 0.025 & 1.98 & \\
\hline 17.754 & 37616 & 1009 & 0.027 & 0.838 & \\
\hline 17.858 & 14024 & 443 & 0.032 & 0.312 & \\
\hline 18.164 & 29164 & 867 & 0.030 & 0.650 & \\
\hline 18.690 & 94405 & 3877 & 0.041 & 2.10 & Aroclor -1248 \\
\hline 18.955 & 44461 & 1013 & 0.023 & 0.991 & \\
\hline 19.100 & 10737 & 346 & 0.032 & 0.239 & \\
\hline 19.332 & 27572 & 887 & 0.032 & 0.614 & \\
\hline 19.522 & 22629 & 822 & 0.036 & 0.504 & \\
\hline 19.682 & 48950 & 1809 & 0.037 & 1.09 & Aroclor -1248 \\
\hline 19.882 & 53507 & 1425 & 0.027 & 1.19 & \\
\hline 20.305 & 46044 & 974 & 0.021 & 1.02 & \\
\hline 20.391 & 25396 & 845 & 0.033 & 0.566 & \\
\hline 20.625 & 13171 & 411 & 0.031 & 0.293 & \\
\hline 20.732 & 15397 & 460 & 0.030 & 0.343 & \\
\hline 20.917 & 25499 & 836 & 0.033 & 0.568 & \\
\hline 21.357 & 17153 & 535 & 0.031 & 0.382 & \\
\hline
\end{tabular}


Data File: /chem/gc2.i/052198.b/031f0401.d

Report Date: 28-May-1998 11:08

\begin{tabular}{|c|c|c|c|c|c|}
\hline$=\begin{array}{c}\mathrm{RT} \\
===\end{array}$ & $==\frac{\mathrm{AREA}}{=======}=$ & $\begin{array}{l}\text { HEIGHT } \\
========\end{array}$ & $\begin{array}{l}\text { HT / AREA } \\
======\end{array}$ & $\begin{array}{l}\% \text { AREA } \\
=======\end{array}$ & $\begin{array}{l}\text { Compounds } \\
===========1=========\end{array}$ \\
\hline 21.522 & 99198 & 3550 & 0.036 & 2.21 & \\
\hline 21.758 & 95718 & 3452 & 0.036 & 2.13 & Aroclor -1248 \\
\hline 22.077 & 25368 & 1157 & 0.046 & 0.565 & \\
\hline 22.238 & 28862 & 882 & 0.031 & 0.643 & \\
\hline 22.451 & 12267 & 527 & 0.043 & 0.273 & \\
\hline 22.601 & 81698 & 1906 & 0.023 & 1.82 & \\
\hline 22.843 & 187106 & 6395 & 0.034 & 4.17 & Aroclor -1254 \\
\hline 23.124 & 33555 & 1437 & 0.043 & 0.748 & \\
\hline 23.387 & 30489 & 1148 & 0.038 & 0.679 & \\
\hline 23.556 & 17613 & 508 & 0.029 & 0.392 & \\
\hline 23.722 & 14530 & 486 & 0.033 & 0.324 & \\
\hline 23.991 & 51838 & 1687 & 0.033 & 1.16 & \\
\hline 24.256 & 72842 & 2630 & 0.036 & 1.62 & \\
\hline 24.499 & 24948 & 858 & 0.034 & 0.556 & \\
\hline 24.781 & 178519 & 5491 & 0.031 & 3.98 & Aroclor-1254 \\
\hline 25.029 & 31764 & 725 & 0.023 & 0.708 & \\
\hline 25.403 & 33071 & 1098 & 0.033 & 0.737 & \\
\hline 25.624 & 27869 & 989 & 0.035 & 0.621 & \\
\hline 25.833 & 19697 & 782 & 0.040 & 0.439 & \\
\hline 26.096 & 203944 & 5157 & 0.025 & 4.54 & \\
\hline 26.922 & 16578 & 640 & 0.039 & 0.369 & \\
\hline 27.246 & 88856 & 3333 & 0.038 & 1.98 & \\
\hline 27.559 & 102105 & 2630 & 0.026 & 2.28 & \\
\hline 27.966 & 33035 & 1083 & 0.033 & 0.736 & \\
\hline 28.508 & 14160 & 497 & 0.035 & 0.316 & \\
\hline 28.796 & 132741 & 4453 & 0.034 & 2.96 & \\
\hline 28.949 & 18937 & 641 & 0.034 & 0.422 & \\
\hline 29.285 & 21127 & 521 & 0.025 & 0.471 & \\
\hline 29.670 & 18936 & 447 & 0.024 & 0.422 & \\
\hline 30.178 & 61627 & 1492 & 0.024 & 1.37 & \\
\hline 30.347 & 35260 & 1127 & 0.032 & 0.786 & \\
\hline 30.992 & 14522 & 464 & 0.032 & 0.324 & \\
\hline 31.590 & 20917 & 721 & 0.034 & 0.466 & \\
\hline 32.454 & 30842 & 947 & 0.031 & 0.687 & \\
\hline 34.136 & 15771 & 535 & 0.034 & 0.351 & \\
\hline 42.230 & 12802 & 254 & 0.020 & 0.285 & \\
\hline 42.719 & $625254(-)$ & 14049 & 0.022 & 13.9 & Decachlorobiphenyl \\
\hline 43.325 & $16481(-)$ & 245 & 0.015 & 0.367 & \\
\hline
\end{tabular}

Total area $=4487933$

$-1026,596$

$-641,735$

$2,824,602$

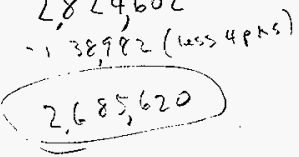


Data File: /chem/gc2.i/052198.b/031f0401.d

Report Date: 29-May-1998 13:25

\section{2-S Laboratory}

Data file: /chem/gc2.i/052198.b/031f0401.d
Lab Smp Id: S985000102
Inj Date : 22-MAY-1998 23:03
Operator : Gerald Ross
Smp Info : $982 \mathrm{Tk} 103 / 100$
Client Smp ID: $982 \mathrm{Tk} 103 / 100$
Misc Info: $982 \mathrm{Tk} 103 / 100$
Comment :
Method : /chem/gc2.i/052198.b/rcrapcb.m
Meth Date : 29-May-1998 13:08 gar Quant Type: ESTD
Cal Date : 22-MAY-1998 20:13 Cal File: 028f0401.d
Als bottle: 1
Dil Factor: 100.000
Integrator: HP Genie
Target version: 3.10
Inst ID: $g c 2 . \mathbf{i}$

\begin{tabular}{|c|c|c|c|c|c|c|}
\hline & & & CONCENT & ATIONS & & \\
\hline & & & $\mathrm{ON}=\mathrm{COL}$ & FINAL & & \\
\hline RT & EXP RT DLT RT & RESPONSE & ( ng) & (ug/Kg) & TARGEI RANGE & RATIO \\
\hline$=$ & 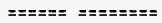 & $\Rightarrow=n=\pi==$ & 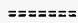 & =ニニ=ニ== & 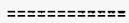 & $====$ \\
\hline & clor $=1016$ & & & CAS & $12674-11-2$ & \\
\hline
\end{tabular}

Peaks not detected for Quant. or Qual. signal(s).

......-.

23 Aroclor -1221

CAS \#: $11104-28-2$

Peaks not detected for Quant, or Qual, signal(s).

24 Aroclor -1232

CAS \#: $1114-16-5$

Peaks not detected for Quant. or Qual. signal(s).

25 Aroclor 1242

CAS \# : $53469-21-9$

Peaks not detected for Quant. or Qual. signal(s).

\begin{tabular}{|c|c|c|c|c|c|c|c|}
\hline \multicolumn{4}{|c|}{26 Aroclor -1248} & \multicolumn{4}{|c|}{ CAS \#: $12672-29-6$} \\
\hline 18.690 & 18.675 & 0.015 & 94404 & 0.135 & 25200 & & 100.00 \\
\hline 19.682 & 19.679 & 0.003 & 48949 & 0.0754 & 14100 & $95.00-135.00$ & 51.85 \\
\hline 21.758 & 21.732 & 0.026 & 95718 & 0.112 & 20900 & $108.00-148.00$ & 101.39 \\
\hline \multicolumn{5}{|c|}{ Average of Peak Concentrations = } & \multicolumn{3}{|l|}{20000} \\
\hline \multicolumn{5}{|c|}{27 Aroclor -1254} & \multicolumn{3}{|c|}{ CAS \#: $11097-69-1$} \\
\hline 21.758 & 21.767 & -0.009 & 95718 & 0.117 & 21900 & & 100.00 \\
\hline 22.843 & 22.846 & -0.003 & 187106 & 0.173 & 32300 & $112.00-152.00$ & 195.48 \\
\hline 24.781 & 24.780 & 0.001 & 178519 & 0.184 & 34400 & $96.00-136.00$ & 186.51 \\
\hline \multicolumn{5}{|c|}{ Average of Peak $C$} & \multicolumn{3}{|l|}{29500} \\
\hline
\end{tabular}


Data File: /chem/gc2.i/052198.b/031f0401.a

Report Date: 29-May-1998 13:25

CONCENTRATIONS

ON-COL FJNAL

RT EXP RT OLT RT RESPONSE ( $\mathrm{ng})(\mathrm{ug} / \mathrm{Kg})$ TARGET RANGE RATIO

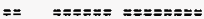

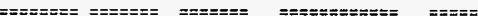

28 Aroctor -1260

CAS \#: $11096-82-5$

Peaks not detected for Quant. or Qual. signal(s).

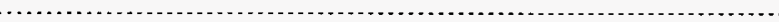

$\$ 29$ Tetrachloro-m-xylene CAS \#: 877-09-8

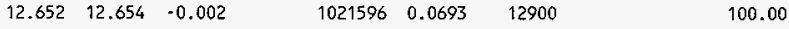

$\$ 30$ Decachlorobiphenyl

CAS \#: 2051-24-3

$42.719 \quad 42.728 \quad-0.009$

$625254 \quad 0.0768$

14300

100.00 
Data File: /chem/gc2.i/052198.b/031f0401.d

Report Date: 29-May-1998 13:25

\section{2-S Laboratory}

\section{RECOVERY REPORT}

Client Name:

Sample Matrix: SoLID

Client SDG: 052198

Lab Smp Id: $\$ 985000102$

Fraction: PEST

Level: LOW

Client Smp ID: 982Tk103/100

Data Type: GC MULTI COMP

Spikelist File: RESOLUTION.spk

Method File: /chem/gc2.i/052198.b/rarapcb.m

Operator: Gerald Ross

SampleType: SAMPLE

Quant Type: ESTD

Misc Info: $982 \mathrm{Tk} 103 / 100$

\begin{tabular}{|c|c|c|c|c|}
\hline SURROGATE COMPOUND & $\begin{array}{l}\text { CONC } \\
\text { ADDED } \\
\text { ug/Kg }\end{array}$ & $\begin{array}{l}\text { CONC } \\
\text { RECOVERED } \\
\text { UG } / \mathrm{Kq}\end{array}$ & $\stackrel{\%}{\circ}$ & LIMITS \\
\hline $\begin{array}{lll}\$ & 29 & \text { Tetrachloro-m-xyle } \\
\$ & 30 \text { Decachlorobiphenyl }\end{array}$ & $\begin{array}{l}14900 \\
14900\end{array}$ & $\begin{array}{l}12900 \\
14300\end{array}$ & $\begin{array}{l}86.60 \\
96.07\end{array}$ & $\begin{array}{l}50-150 \\
50-150\end{array}$ \\
\hline
\end{tabular}


Data File: /chem/gc2.i/052198,b/031f0401.d HNF-SD-WM-DP-306 REV. O Page 4 Report Date: 29-May-1998 13:25

\section{2-S Laboratory}

\section{TARGET COMPOUNDS}

Client Name:

Lab Smp Id: S985000102

Sample Location:

Sample Date:

Sample Matrix: Soll

Analysis Type: PEST

Data Type: GC MULTI COMP

Misc Info: $982 \mathrm{Tk} 103 / 100$
Client SDG: 052198

Client Smp ID: $982 \mathrm{Tk} 103 / 100$

Sample Point:

Date Received:

Quant Type: ESTD

Level: LOW

Operator: Gerald Ross

CAS NO.

COMPOUND

CONCENTRATION UNITS:

( $\mathrm{ug} / \mathrm{L}$ or $\mathrm{ug} / \mathrm{KG}$ ) $\mathrm{ug} / \mathrm{Kg}$

$Q$

\begin{tabular}{|c|c|c|}
\hline $12674-11-2-----$ Aroclor -1016 & 3300 & $\mathrm{U}$ \\
\hline $11104-28-2-\cdots--$ Aroclor -1221 & 6600 & $\mathrm{U}$ \\
\hline $1114-16-5-----$ Aroclor -1232 & 3300 & $\mathrm{U}$ \\
\hline $53469-21-9-\cdots$ Aroclor-1242 & 3300 & $U$ \\
\hline $12672-29-6----$ Aroclor -1248 & 3300 & $\mathrm{UX}$ \\
\hline $11097-69-1-----$ Aroclor -1254 & 47700 & \\
\hline $11096-82-5-\ldots-$ Aroclor -1260 & 3300 & U \\
\hline 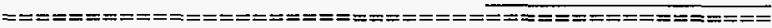 & 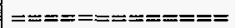 & $=\approx===$ \\
\hline $877-09-8-\cdots-----$ Tetrachloro-m-xylene & 12900 & \\
\hline 2051-24-3-n-Decachlorobiphenyl & 14300 & \\
\hline
\end{tabular}


Data File: /chem/gc2, i/052198,b/031fo401,d

Date : 22-MAY-1998 23:03

Client ID: 982Tk103/100

Sample Info: $982 T k 103 / 100$

Volume Injected $(\mathrm{uL}) \div 1.0$

Instrument: $\mathrm{gc} 2.1$

Column phase: Xti-5

Operator: Gerald Ross

Column diameter: 0.25

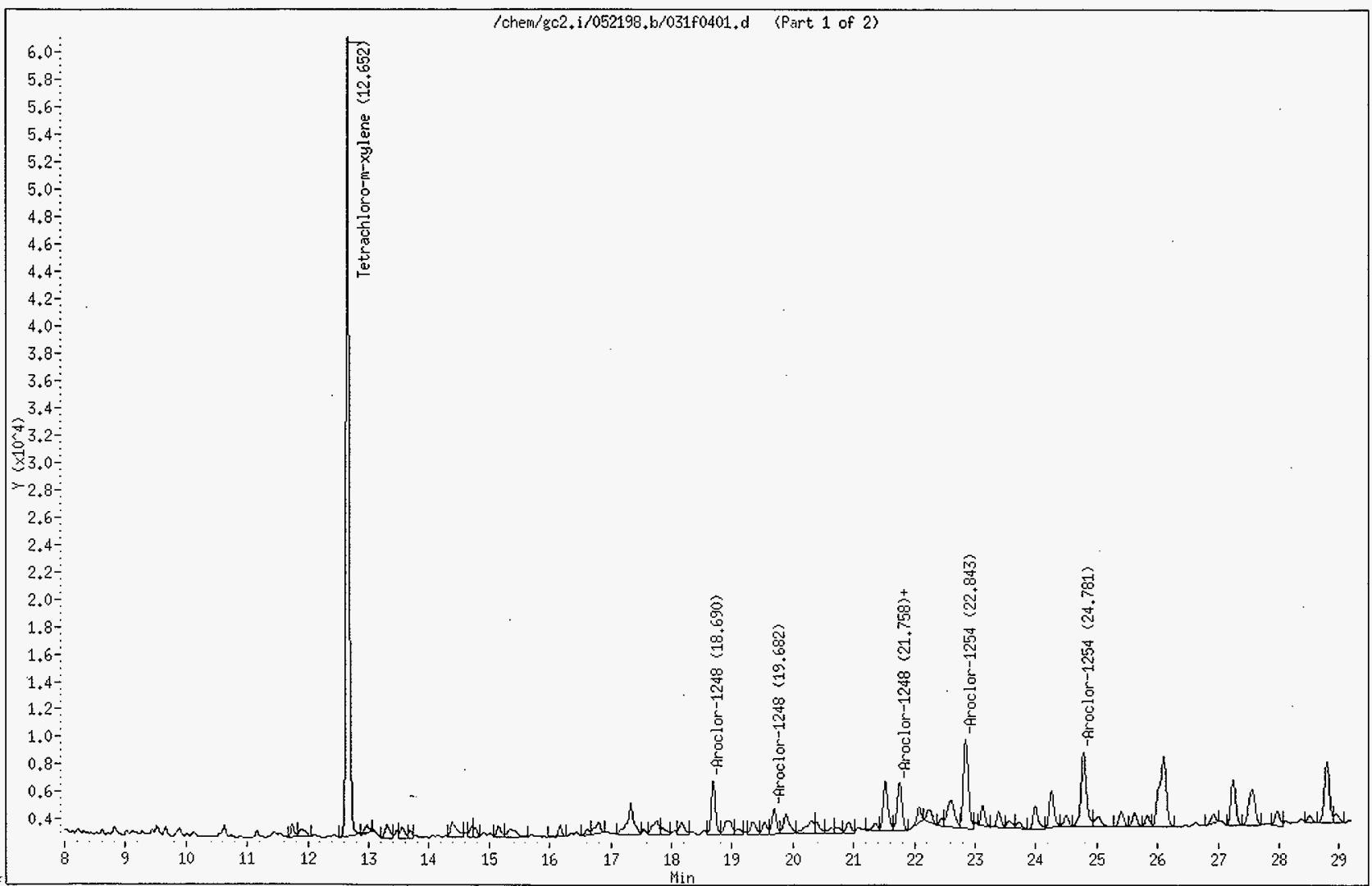


Data File: /chen/gc2, i/052198,b/031fo401,d

Page 6

Date : 22-HAY-1998 23:03

Client ID: 982Tk103/100

Sample Info: 982Tk103/100

Volume Injected $(u L): 1,0$

Instrument: $\mathrm{gc2}+\mathrm{i}$

Column phase: Xti-5

Operator: Gerald Ross

Column diameter: 0.25

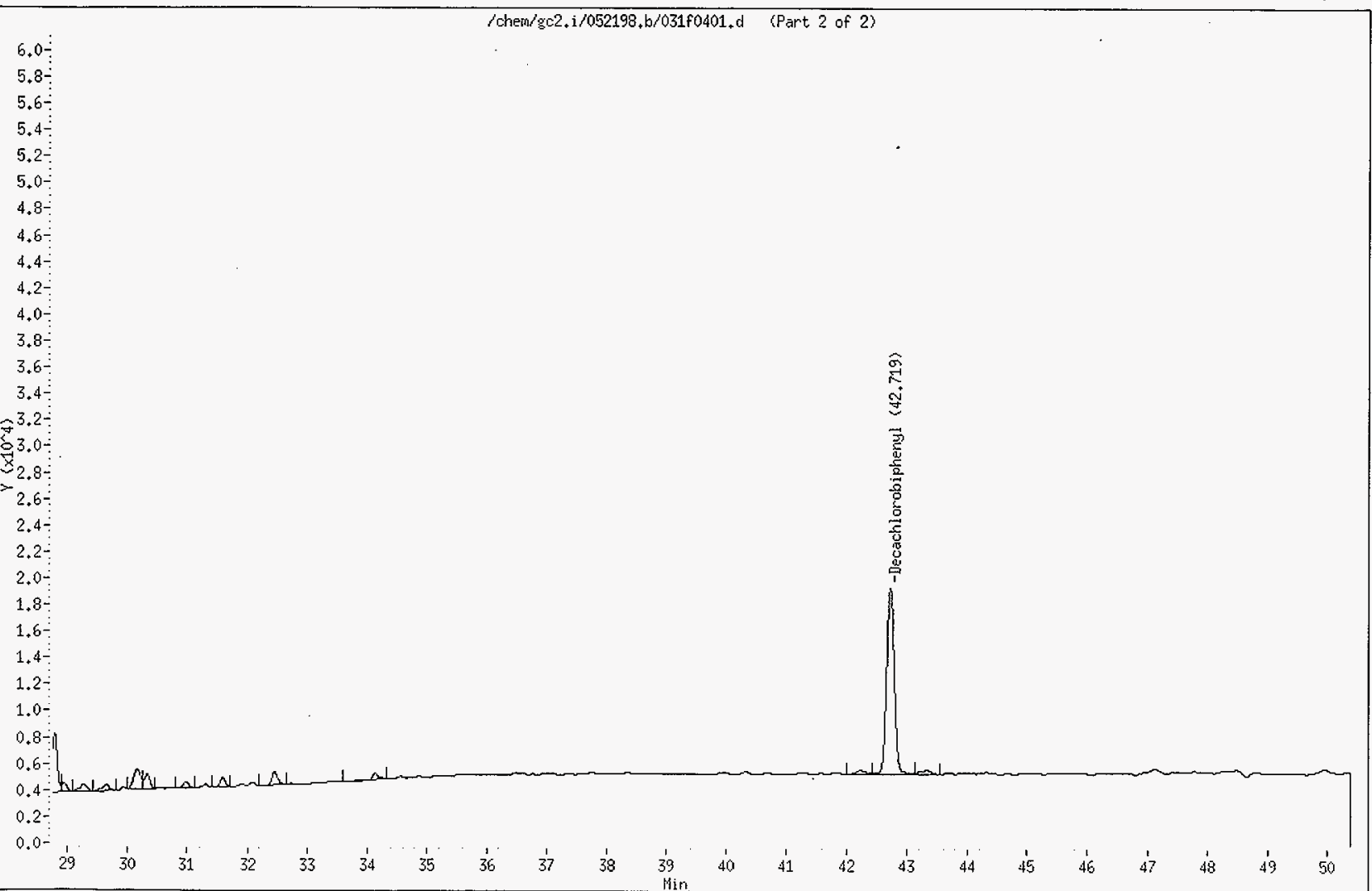


Data File: /chem/gc2.i/052198.b/033f0401.d

Page 1

Report Date: 28-May-1998 11:09

$$
222-S \text { Laboratory }
$$

Data file : /chem/gc2.i/052198.b/033f0401.d
Lab Smp Id: $984 \mathrm{Tk} 103 / 100$
Client Smp ID: $984 \mathrm{Tk} 103 / 100$
Inj Date : 23-MAY-98 00:56
operator : Gerald Ross
Smp Info : 984Tk103/100
Inst ID: $9 \mathrm{C} 2 . \mathrm{i}$
Misc Info : $984 \mathrm{Tk} 103 / 100$
comment :
Method : /chem/gc2.i/052198.b/rcrapcb.m
Meth Date : 28-May-1998 11:08
Cal Date : 22-MAY-98 20:13
Quant TYpe: AREA\%
Als bottle: 1
Dil Factor: 100.000
Integrator: HP Genie
Target Version: 3.10
Cal File: 028f0401.d
Compound sublist: Aroclors.sub Sample Matrix: SoIL

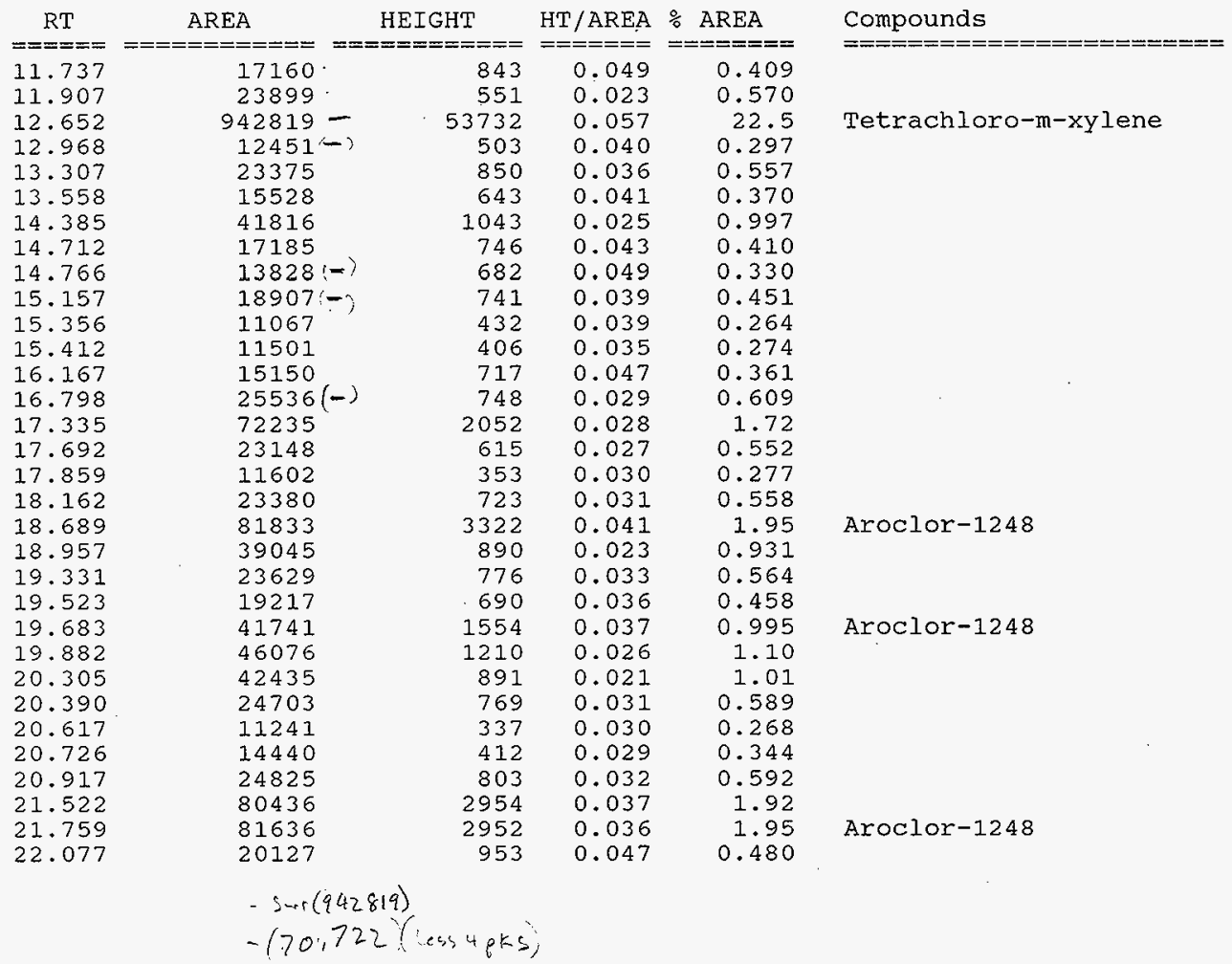


HNF-SD-WMi-DP-306 REV. 0

Data File: /chem/gc2.i/052198.b/033f0401.d

Report Date: 28-May-1998 11:09

\begin{tabular}{|c|c|c|c|c|c|}
\hline $\mathrm{RT}$ & $==\stackrel{\text { AREA }}{======}$ & HEIGHT & HT / AREA & $\%$ AREA & Compounds \\
\hline 22.237 & 23202 & 722 & 0.031 & 0.553 & \\
\hline 22.600 & 67161 & 1551 & 0.023 & 1.60 & \\
\hline 22.843 & 160935 & 5506 & 0.034 & 3.84 & Aroclor -1254 \\
\hline 23.124 & 28395 & 1220 & 0.043 & 0.677 & \\
\hline 23.387 & 25154 & 948 & 0.038 & 0.600 & \\
\hline 23.555 & 14571 & 420 & 0.029 & 0.348 & \\
\hline 23.722 & 12198 & 397 & 0.033 & 0.291 & \\
\hline 23.991 & 43937 & 1444 & 0.033 & 1.05 & \\
\hline 24.257 & 61598 & 2219 & 0.036 & 1.47 & \\
\hline 24.499 & 21762 & 725 & 0.033 & 0.519 & \\
\hline 24.780 & 148448 & 4570 & 0.031 & 3.54 & Aroclor -1254 \\
\hline 25.028 & 24032 & 551 & 0.023 & 0.573 & \\
\hline 25.404 & 28013 & 920 & 0.033 & 0.668 & \\
\hline 25.623 & 23975 & 836 & 0.035 & 0.572 & \\
\hline 25.832 & 16165 & 627 & 0.039 & 0.386 & \\
\hline 26.096 & 171832 & 4232 & 0.025 & 4.10 & \\
\hline 26.922 & 19024 & 630 & 0.033 & 0.454 & \\
\hline 27.246 & 73599 & 2784 & 0.038 & 1.76 & \\
\hline 27.557 & 91040 & 2166 & 0.024 & 2.17 & \\
\hline 27.966 & 27810 & 888 & 0.032 & 0.663 & \\
\hline 28.506 & 11139 & 397 & 0.036 & 0.266 & \\
\hline 28.795 & 109477 & 3729 & 0.034 & 2.61 & \\
\hline 28.948 & 17219 & 546 & 0.032 & 0.411 & \\
\hline 29.285 & 21757 & 471 & 0.022 & 0.519 & \\
\hline 29.669 & 19597 & 392 & 0.020 & 0.467 & \\
\hline 30.179 & 45258 & 1119 & 0.025 & 1.08 & \\
\hline 30.349 & 32580 & 962 & 0.030 & 0.777 & \\
\hline 30.987 & 58129 & 565 & 0.010 & 1.39 & \\
\hline 31.588 & 15524 & 555 & 0.036 & 0.370 & \\
\hline 32.449 & 44172 & 891 & 0.020 & 1.05 & \\
\hline 33.583 & 20118 & 169 & 0.008 & 0.480 & \\
\hline 34.134 & 19633 & 452 & 0.023 & 0.468 & \\
\hline 35.201 & 24266 & 263 & 0.011 & 0.579 & \\
\hline 37.800 & 124658 & 1371 & 0.011 & 2.97 & \\
\hline 38.307 & 43256 & 367 & 0.008 & 1.03 & \\
\hline 39.987 & 12759 & 154 & 0.012 & 0.304 & \\
\hline 41.228 & 39493 & 243 & 0.006 & 0.942 & \\
\hline 42.228 & 26566 & 331 & 0.012 & 0.634 & \\
\hline 42.719 & 533669 & 11738 & 0.022 & 12.7 & Decachlorobiphenyl \\
\hline 43.316 & 18898 & 252 & 0.013 & 0.451 & \\
\hline
\end{tabular}

Total area $=4192986$

$-742,819$

$-5 \$ 2,567$

2697,600

$$
\frac{-70,222}{2,626,878}
$$


Data File: /chem/gc2.i/052198.b/033f0401.d

Report Date: 29-May-1998 13:25

\section{2-S Laboratory}

Data file : /chem/gc2.i/052198.b/033f0401.d
Lab Smp Id: S98S000102D
Inj Date : 23-MAY-1998 00:56
operator : Gerald Ross
Client Smp ID: $984 \mathrm{Tk} 103 / 100$
Smp Info: $984 \mathrm{Tk} 103 / 100$
Misc Info: $984 \mathrm{Tk} 103 / 100$
Comment :
Method : /chem/gc2.i/052198.b/xcrapcb.m
Meth Date : 29-May-1998 13:08 gar quant Type: ESTD
Cal Date : 22-MAY-1998 20:13 Cal File: 028f0401.d
Als bottle: 1
Dil Factor: 100.000
Integrator: HP Genie
Target Version: 3.10
Inst ID: $g c 2 . i$
Compound Sublist: Aroclors. sub Sample Matrix: SOIL

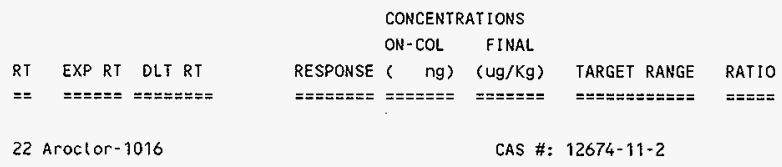

Peaks not detected for Quant. or Qual. signal(s).

23 Aroclor 1221

CAS \#: $11104-28-2$

Peaks not detected for Quant. or Qual, signal(s).

24 Aroclor -1232 CAS \#: $1114-16-5$

Peaks not detected for Quant. or Qual. signal(s).

......

25 Aroclor -1242

CAS \#: $53469-21-9$

Peaks not detected for Quant. or Qual. signal(s).

\begin{tabular}{|c|c|c|c|c|c|c|c|}
\hline \multicolumn{4}{|c|}{26 Aroclor- 1248} & \multicolumn{4}{|c|}{ CAS \#: $12672-29-6$} \\
\hline 18.689 & 18.675 & 0.014 & 81833 & 0.117 & 21000 & & 100.00 \\
\hline 19.683 & 19.679 & 0.004 & 41740 & 0.0643 & 11500 & $95.00-135.00$ & 51.01 \\
\hline 21.759 & 21.732 & 0.027 & 81635 & 0.0956 & 17100 & $108.00-148.00$ & 99.76 \\
\hline \multicolumn{5}{|c|}{ Average of Peak Con } & \multicolumn{3}{|l|}{16500} \\
\hline \multicolumn{5}{|c|}{27 Aroclor -1254} & \multicolumn{3}{|c|}{ CAS \#: $11097-69-1$} \\
\hline 21.759 & 21.767 & -0.008 & 81635 & 0.100 & 17900 & & 100.00 \\
\hline 22.843 & 22.846 & -0.003 & 160935 & 0.149 & 26700 & $112.00-152.00$ & 197.14 \\
\hline 24.780 & 24.780 & 0.000 & 148448 & 0.153 & 27500 & $96.00-136.00$ & 181.84 \\
\hline \multicolumn{5}{|c|}{ Average of Peak } & \multicolumn{3}{|l|}{24000} \\
\hline
\end{tabular}


Data File: /chem/gc2.i/052198.b/033f0401.d Report Date: 29-May-1998 13:25

CONCENTRATIONS

ON-COL FINAL

RT EXP RT DLT RT

RESPONSE ( $\mathrm{ng}$ ) (Ug/ $\mathrm{Kg}$ )

TARGET RANGE RATIO

$==\quad== \pm==0== \pm= \pm= \pm=0$

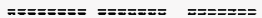

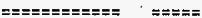

28 Aroclor -1260

CAS \#: $11096-82 \cdot 5$

Peaks not detected for Quant. or Qual. signal(s).

$\$ 29$ retrachloro-m-xylene

CAS \#: $877-09-8$

$12.652 \quad 12.654 \quad-0.002$

$942818 \quad 0.0639 \quad 11400$

100.00

$\$ 30$ Decachlorobiphenyl

CAS \# : 2051-24-3

$\begin{array}{lll}42.719 & 42.728 & -0.009\end{array}$

5336690.0656

11700

100.00

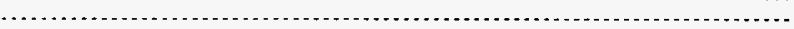


Data File: /chem/gc2.i/052198.b/033f0401.d Report Date: 29-May-1998 13:25

\section{2-S Laboratory \\ TARGET COMPOUNDS}

Client Name:

Lab Smp Id: S98S000102D

Sample Location:

Sample Date:

Sample Matrix: SOIL

Analysis Type: PEST

Data Type: GC MULTI COMP

Misc Info: $984 \mathrm{Tk} 103 / 100$
Client SDG: 052198

Client Smp ID: $984 \mathrm{Tk} 103 / 100$

Sample Point:

Date Received:

Quant Type: ESTD

Level: LOW

Operator: Gerald Ross

CONCENTRATION UNITS:

( $\mathrm{ug} / \mathrm{L}$ or $\mathrm{ug} / \mathrm{KG}$ ) $\mathrm{ug} / \mathrm{Kg}$

\begin{tabular}{|c|c|c|}
\hline 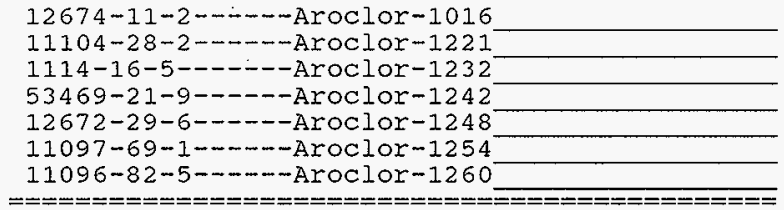 & $\begin{array}{r}3300 \\
6600 \\
3300 \\
3300 \\
3300 \\
44800 \\
3300 \\
===-==-=\end{array}$ & $\begin{array}{l}\mathrm{U} \\
\mathrm{U} \\
\mathrm{U} \\
\mathrm{UX} \\
\mathrm{U} \\
===\end{array}$ \\
\hline $\begin{array}{l}877-09-8-------T e t r a c h l o r o-m-x y l e n e \\
2051-24-3-------D e c a c h l o r o b i p h e n y l\end{array}$ & $\begin{array}{l}11400 \\
11700\end{array}$ & \\
\hline
\end{tabular}

* Per LA-523-136 Option 2 (Total Area) 
Data File: /chem/gc2.i/052198.b/033f0401.d

Report Date: 29-May-1998 13:25

Page 3

222-s Laboratory

RECOVERY REPORT

Client Name:

Sample Matrix: SOLID

Lab Smp Id: S98S000102D

Level: LOW

Data Type: GC MULTI COMP

Spikelist File: RESOLUTION, spk

Method File: /chem/gc2.i/052198.b/rcrapcb.m

Client SDG: 052198 .

Fraction: PEST

Client Smp ID: $984 \mathrm{Tk} 103 / 100$

Operator: Gerald Ross

SampleType: SAMPLE

Quant Type: ESTD

Misc Info: $984 \mathrm{Tk} 103 / 100$

\begin{tabular}{|c|c|c|c|c|}
\hline SURROGATE COMPOUND & $\begin{array}{l}\text { CONC } \\
\text { ADDED }\end{array}$ & $\begin{array}{c}\text { CONC } \\
\text { RECOVERED }\end{array}$ & $\stackrel{\%}{\%}$ RECOVERED & LIMITS \\
\hline $\begin{array}{lll}\$ & 29 & \text { Tetrachloro-m-xyle } \\
\$ & 30 \text { Decachlorobiphenyl }\end{array}$ & $\begin{array}{l}14300 \\
14300\end{array}$ & $\begin{array}{l}11400 \\
11700\end{array}$ & $\begin{array}{l}79.93 \\
82.00\end{array}$ & $\begin{array}{l}50-150 \\
50-150\end{array}$ \\
\hline
\end{tabular}




$$
\text { 222-S Laboratory }
$$

Data file: /chem/gc2.i/052198.b/036f0401.d

Lab Smp Id: $986 \mathrm{Tk} 103 \mathrm{MS} / 200$

Inj Date : 23-MAY-98 03:46

operator : Gerald Ross

Smp Info : 986Tk103MS/200

Misc Info : $986 \mathrm{Tk} 103 \mathrm{MS} / 200$

Comment

Method

Meth Date : 28-May-1998 11:08

Cal Date : 22-MAY-98 20:13

Als bottle: 1

Dil Factor: 200.000

Integrator: HP Genie

Target Version: 3.10

RT
$=====$
11.738
12.654
13.308
14.388
14.715
15.158
15.407
16.169
16.799
17.337
17.756
18.164
18.688
18.907
19.331
19.687
19.884
20.299
20.392
20.921
21.358
21.523
21.762
22.085
22.239
22.565
22.843
23.125
23.389
23.724
23.993
24.258

AREA

12817
553999
11111
29939
22880
11192
14792
10319
15786
47411
24605
16272
95548
40157
12915
45056
24960
27576
12646
12222
13129
83388
126964
21000
12048
63626
193561
46797
16080
13426
49617
86939

HEIGHT

$======$

\section{1}

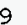

0

$2-$

\section{9}

-

678

32158

471

630

583

441

297

465

473

1329

652

468

4008

974

480

1842

736

707

466

515

535

3175

4730

930

387

1478

6901

1989

587

461

1890

3245
Client Smp ID: 986Tk103MS/200 Inst ID: $\mathrm{gC2} . \mathrm{i}$
Muant Type: AREAs

Cal File: 028f0401.d

QC Sample: MS

Compound Sublist: Aroclors.sub Sample Matrix: SOIL
HT/AREA \% AREA

$===========$

0.053

0.058

0.042

0.021

0.025

0.039

0.020

0.045

0.030

0.028

0.026

0.029

0.042

0.024

0.037

0.041

0.029

0.026

0.037

0.042

0.041

0.038

0.037

0.044

0.032

0.023

0.036

0.043

0.037

0.034

0.038

0.037
0.386

16.7

0.334

0.901

0.688

0.337

0.445

0.310

0.475

1.43

0.740

0.490

2.87

1. 21

0.389

1.36

0.751

0.830

0.380

0.368

0.395

2.51

3.82

0.632

0.362

1.91

5.82

1.41

0.484

0.404

1.49

2.62
Compounds

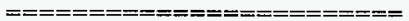

Tetrachloro-m-xylene

Aroclor -1248

Aroclor -1248

Aroclor -1248

Aroclor -1254

Non PCB

580,977 
Data File: /chem/gc2.i/052198.b/036f0401.d Report Date: 28-May-1998 11:09

Page 2

$== \pm T$

24.502

24.779

25.029

25.407

25.624

25.834

26.098

26.923

27.249

27.559

27.968

28.509

28.798

28.948

29.280

29.674

30.181

30.350

30.993

31.590

32.454

34.139

35.573

37.792

39.430

42.725

44.357

AREA

$===ニ====$

26149

16697

37051

28884

17306

218166

27334

88800

106548

23952

13138

140198

19085

16485

13932

30678

37062

11249

20806

21813

12586

11798

40435

15143

$351885-1$

16930 ( )
HEIGHT

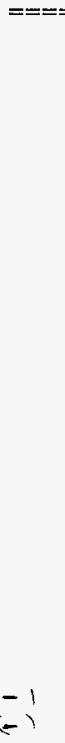

Total area $=3,323,438$

$$
\begin{array}{ll}
p^{\prime} & -580,777 \\
p^{2} & -368,815 \\
\hline 2,373,646
\end{array}
$$

HT/AREA \% AREA

$======$

0.037

0.033

0.023

0.033

0.035

0.039

0.024

0.028

0.038

0.026

0.039

0.037

0.034

0.036

0.028

0.026

0.025

0.032

0.034

0.035

0.035

0.041

0.009

0.012

0.005

0.023

0.009
0.787

5.73

0.502

1.11

0.869

0.521

6.56

0.822

2.67

3.20

0.721

0.395

4.22

0.574

0.496

0.419

0.923

1. 12

0.338

0.626

0.656

0.379

0.355

1.22

0.456

10.6

0.509
Compounds

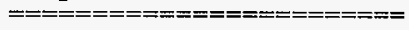

Aroclor -1254

Decachlorobiphenyl 
Data File:/chem/gc2.i/052198.b/036f0401.d Report Date: 29-May-1998 11:32

\section{Page 3}

\section{2-S Laboratory}

\section{RECOVERY REPORT}

Client Name:

Sample Matrix: SOLID

Lab Smp Id: $\$ 985000102 \mathrm{MS}$

Level: LoW

Data Type: GC MULTI COMP

Spikelist File: Aroclor-1254.spk Method File: / chem/gc2.i/052198.b/rcrapcb.m

Misc Info: 986Tk103MS/200
Client SDG: 052198

Fraction: PEST

Client Smp ID: 986Tk103MS/200

Operator: Gerald Ross

SampleType: MS

Quant Type: ESTD

\begin{tabular}{|c|c|c|c|c|}
\hline SPIKE COMPOUND & $\begin{array}{l}\text { CONC } \\
\text { ADDED } \\
\text { ug/Kg }\end{array}$ & $\begin{array}{c}\text { CONC } \\
\text { RECOVERED } \\
\text { ug } / \mathrm{Kg}\end{array}$ & $\begin{array}{c}\% \\
\text { RECOVERED }\end{array}$ & LIMITS \\
\hline 27 Aroclor-1254 & 45200 & -64200 & $-2.141 .91 x$ & $\overline{50-150}$ \\
\hline & $81.82-47.66=34.16$ & $34.16 / 45.25 \times 100$ & $=75.49 \%_{0}$ & , \\
\hline SURROGATE COMPOUND & $\begin{array}{l}\text { CONC } \\
\text { ADDED } \\
\mathrm{ug} / \mathrm{Kg}\end{array}$ & $\begin{array}{c}\text { CONC } \\
\text { RECOVERED } \\
\text { ug/Kg }\end{array}$ & $\begin{array}{c}\% \\
\text { RECOVERED }\end{array}$ & LIMITS \\
\hline $\begin{array}{lll} & 29 & \text { Tetrachloro-m-xyle } \\
\$ & 30 & \text { Decachlorobiphenyl }\end{array}$ & $\begin{array}{l}14500 \\
14500\end{array}$ & $\begin{array}{l}13600 \\
15600\end{array}$ & $\begin{array}{r}93.93 \\
108.13\end{array}$ & $\begin{array}{l}50-150 \\
50-150\end{array}$ \\
\hline
\end{tabular}




\section{2-S Laboratory \\ TARGET COMPOUNDS}

Client Name:

Lab Smp Id: S98S000102MS

Sample Location:

Sample Date:

Sample Matrix: SoIL

Analysis Type: PEST

Data Type: GC MULTI COMP

Misc Info: 986Tk103MS/200
Client SDG: 052198

Client Smp ID: $986 \mathrm{Tk} 103 \mathrm{MS} / 200$

Sample Point:

Date Received:

Quant Type: ESTD

Level: IOW

Operator: Gerald Ross

CAS NO.

COMPOUND

CONCENTRATION UNITS:

( $u g / L$ or $u g / K G$ ) $u g / K g$

\begin{tabular}{|c|c|c|}
\hline $\begin{array}{l}12674-11-2-\cdots-- \text { Aroclor }-1016 \\
11104-28-2-\cdots-- \text { Aroclor }-1221 \\
1114-16-5--\cdots-- \text { Aroclor }-1232 \\
53469-21-9-\cdots-- \text { Aroclor }-1242 \\
12672-29-6 \cdots-- \text { Aroclor }-1248 \\
11097-69-1---- \text { Aroclor }-1254 \\
11096-82-5-\cdots-- \text { Aroclor }-1260\end{array}$ & $\begin{array}{r}6600 \\
13200 \\
6600 \\
6600 \\
6600 \\
81800 \\
6600\end{array}$ & $\begin{array}{l}\mathrm{U} \\
\mathrm{U} \\
\mathrm{U} \\
\mathrm{UX} \\
\mathrm{U}^{*}\end{array}$ \\
\hline 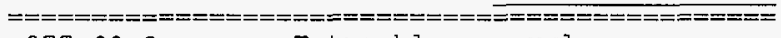 & $===\Rightarrow=======$ & $=====$ \\
\hline $\begin{array}{l}\text { 877-09-8-------Tetrachloro-m-xylene } \\
2051-24-3------D e c a c h \text { lorobiphenyl }\end{array}$ & $\begin{array}{l}13600 \\
15600\end{array}$ & \\
\hline
\end{tabular}




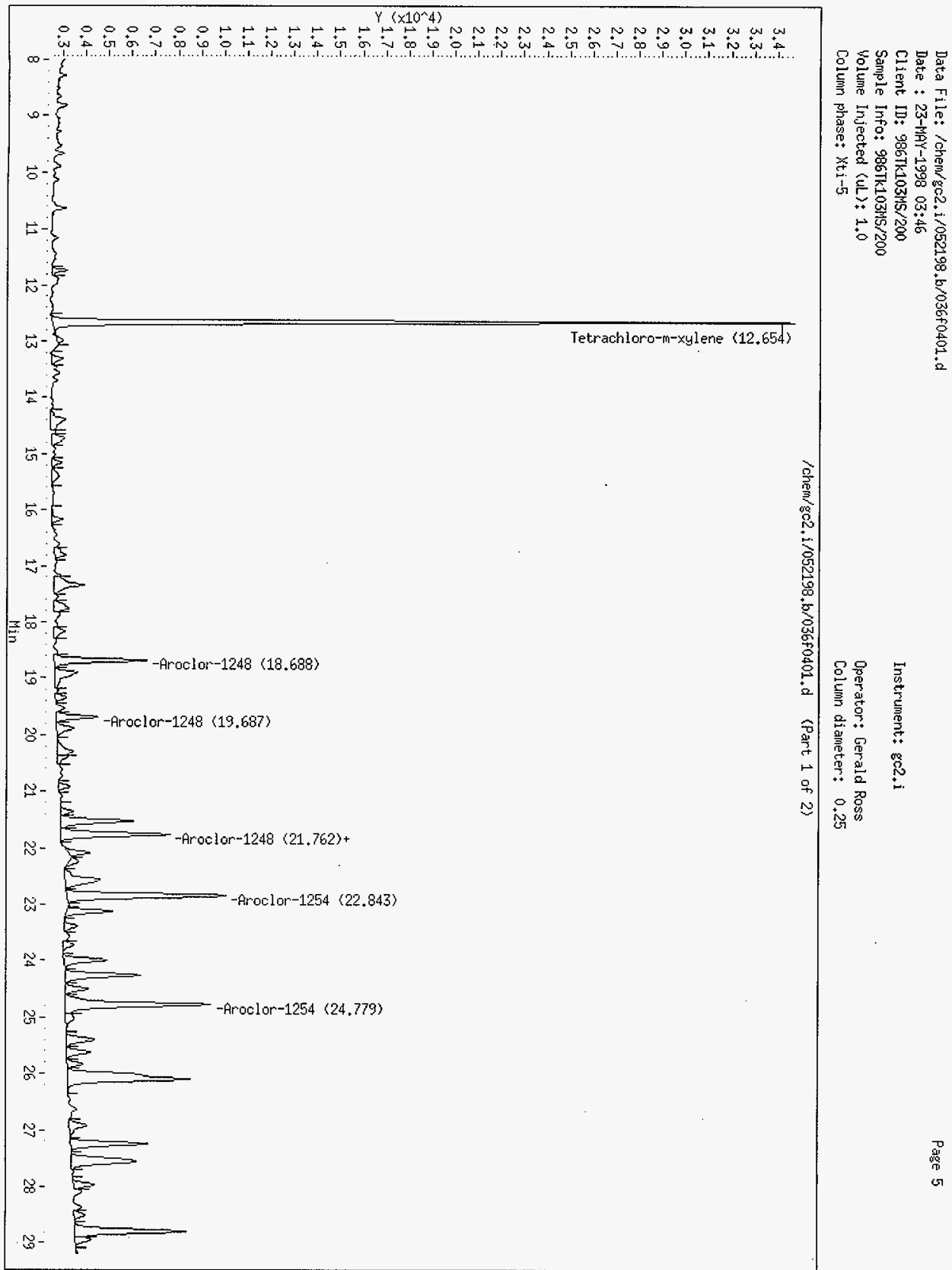


Data File: /chem/gc2, i/052198,b/036fo401.d

Date : $23-\mathrm{NAY}-1998 \quad 03 \div 46$

Client ID: 986Tk103tS/200

Sample Info: 986Tk103MS/200

Volume Injected $(u L) \div 1.0$

Instrument: go2,

Colusin phase: $X_{t i-5}$

Operator: Gerald Ross

Columin diameter: 0.25

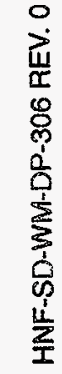

37


Data File: /chem/gc2.i/052198.b/036f0401.d

Report Date: 29-May-1998 13:25

Page 1

\section{2-S Laboratory}

Data file: /chem/gc2.i/052198.b/036f0401.d

Lab Smp Id: S98S000102MS

Inj Date : 23-MAY-1998 03:46

operator : Gerald Ross

Client Smp ID: 986Tk103MS/200

Smp Info : 986Tk103MS/200

Inst ID: $902 . i$

Misc Info : $986 \mathrm{Tk} 103 \mathrm{MS} / 200$

comment

Method

/chem/gc2.i/052198.b/rcrapcb.m

Meth Date : 29-May-1998 13:08 gar

Cal Date : 22-MAY-1998 20:13

Quant Type: ESTD

Als bottle: 1

Dil Factor: 200.000

Integrator: HP Genie

Target Version: 3.10

Cal File: $028 \mathrm{f} 0401 . \mathrm{d}$

QC Sample: MS

Compound Sublist: Aroclors. sub Sample Matrix: SOIL

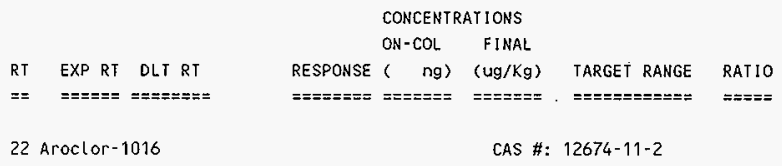

Peaks not detected for Quant. or Qual. signal(s).

23 Aroclor $-1221 \quad$ CAS $\#: 11104-28-2$

Peaks not detected for Quant. Or Qual. Signal(s).

24 Aroclor -1232

CAS \#: $1114-16-5$

Peaks not detected for Quant. or Qual. signal(s).

Peaks not detected for Quant. or Qual. signal(s).

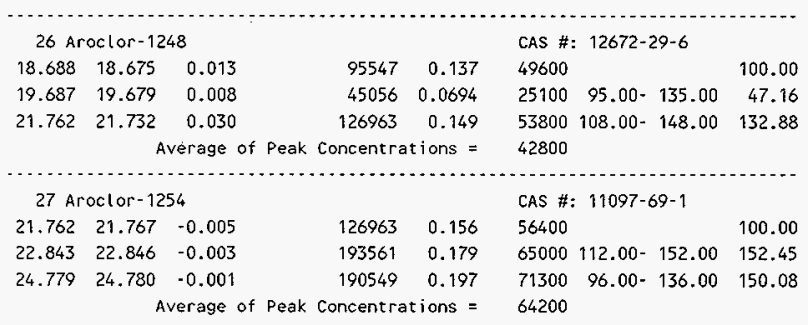


Data File: /chem/gc2.i/052198.b/036f0401.d Report Date: 29-May-1998 13:25

\begin{tabular}{|c|c|c|c|c|c|c|}
\hline \multirow[b]{3}{*}{$R T$} & & \multicolumn{3}{|c|}{ CONCENTRATIONS } & \multirow[b]{3}{*}{ TARGET RANGE } & \multirow[b]{3}{*}{ RATIO } \\
\hline & & & $\mathrm{ON}-\mathrm{COL}$ & FINAL & & \\
\hline & EXP RT DLT RT & RESPONSE & ( $n g$ ) & $(u g / K g)$ & & \\
\hline$==$ & $== \pm= \pm=== \pm====0$ & $==E== \pm==$ & $=======$ & $==\geq=x==$ & 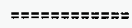 & $====$ \\
\hline
\end{tabular}

Peaks not detected for Quant. or Qual. signal(s).

$\$ 29$ Tetrachloro-m-xylene

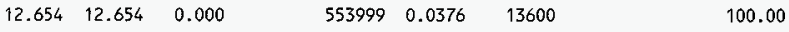

$\$ 30$ Decachlorobiphenyl

CAS \#: 2051-24-3

$\begin{array}{llll}42.725 \quad 42.728 & -0.003\end{array}$

$351885 \quad 0.0432$

15600

100.00 
Data File: /chem/gc2.i/052198.b/038f0401.d Report Date: 28-May-1998 11:09

\section{Page 1}

\section{2-S Laboratory}

Data file:/chem/gc2.i/052198.b/038f0401.d
Lab Smp Id: $988 T k 103 \mathrm{MSD} / 200$
Inj Date : 23-MAY-98 05:40
operator : Gerald Ross
Smp Info: $988 T k 103 \mathrm{MSD} / 200$
Client Smp ID: 988Tk103MSD/200
Misc Info :
comment :
Method : /chem/gc2.i/052198.b/rcrapcb.m
Meth Date : 28-May-1998 11:08
Cal Date : 22-MAY-98 20:13
Als bottle: 1
Dil Factor: 200.000
Integrator: HP Genie
Target Version: 3.10
Inst ID: $9 \mathrm{C} 2 . \mathrm{i}$
Quant Type: AREA\%
Cal File: 028f0401.d
QC Sample: MSD
Compound Sublist: Aroclors.sub
Sample Matrix: SoIL

\begin{tabular}{|c|c|c|c|c|}
\hline$=\begin{array}{c}\mathrm{RT} \\
====\end{array}$ & $\begin{array}{c}\text { AREA } \\
=========\end{array}$ & $\begin{array}{l}\text { HEIGHT } \\
========\end{array}$ & $\begin{array}{l}\mathrm{HT} / \mathrm{AREA} \\
======\end{array}$ & $\begin{array}{l}\% \text { AREA } \\
======\end{array}$ \\
\hline 12.654 & $549168-$ & 32056 & 0.058 & 16 \\
\hline 14.388 & 22486 & 545 & 0.024 & 0.68 \\
\hline 14.773 & $17788(-)$ & 347 & 0.020 & 0.54 \\
\hline 15.363 & 12726 & 254 & 0.020 & 0.3 \\
\hline 16.802 & $14129(-)$ & 421 & 0.030 & 0.42 \\
\hline 17.335 & 33818 & 807 & 0.024 & 1.03 \\
\hline 17.756 & 29029 & 856 & 0.029 & 0.88 \\
\hline 18.171 & 12266 & 315 & 0.026 & 0.37 \\
\hline 18.688 & 93723 & 3940 & 0.042 & 2.8 \\
\hline 18.907 & 37044 & 919 & 0.025 & 1.1 \\
\hline 19.332 & 11865 & 447 & 0.038 & 0.36 \\
\hline 19.686 & 43833 & 1777 & 0.041 & 1.3 \\
\hline 19.884 & 21744 & 653 & 0.030 & 0.66 \\
\hline 20.299 & 26000 & 665 & 0.026 & 0.78 \\
\hline 21.360 & 12817 & 538 & 0.042 & 0.38 \\
\hline 21.523 & 81661 & 3064 & 0.038 & 2.4 \\
\hline 21.762 & 125280 & 4703 & 0.038 & 3.8 \\
\hline 22.085 & 19048 & 904 & 0.047 & 0.57 \\
\hline 22.237 & 11562 & 370 & 0.032 & 0.35 \\
\hline 22.566 & 61863 & 1456 & 0.024 & 1.8 \\
\hline 22.843 & 186526 & 6677 & 0.036 & 5 \\
\hline 23.125 & 45720 & 1931 & 0.042 & 1 \\
\hline 23.390 & 14300 & 524 & 0.037 & 0.4 \\
\hline 23.724 & 13230 & 461 & 0.035 & 0.40 \\
\hline 23.992 & 53138 & 1881 & 0.035 & 1.6 \\
\hline 24.257 & 85006 & 3144 & 0.037 & 2.5 \\
\hline 24.503 & 26420 & 967 & 0.037 & 0.80 \\
\hline 24.778 & 186728 & 6021 & 0.032 & 5.6 \\
\hline 25.029 & 15554 & 349 & 0.022 & 0.47 \\
\hline 25.4 .06 & 36735 & 1216 & 0.033 & 1. \\
\hline 25.626 & 30976 & 1026 & 0.033 & 0.5 \\
\hline 5.835 & 17077 & 641 & 0.038 & \\
\hline
\end{tabular}

Compounds

$========-m=====$
Tetrachloro-m-xylene

14.388

14.773

15.363

16.802

17.335

17.756

18.171

18.688

18.907

19.332

19.686

19.884

21.523

21.762

22.085

22.237

22.566

22.843

23.125

23.390

23.724

23.992

24.257

24.503

24.778

25.4 .06

25.835

17077

641

0.038

0.518

Aroclor -1248

Aroclor -1248

Aroclor -1248

Aroclor -1254

Aroclor -1254

ponper. 581,085 
HNF-SD-WRA-DP-306 REV. 0

Data File:/chem/gc2.i/052198.b/038f0401.d Report Date: 28-May-1998 11:09

$=\stackrel{\mathrm{RT}}{==}=$

26.098

26.922

27.248

27.558

27.968

28.508

28.798

28.948

29.278

29.671

30.181

30.351

30.991

31.592

32.453

33.657

34.139

35.216

37.816

38.299

41.236

42.723

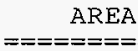

211358

26026

85739

102735

23416

12148

134093

17873

19112

15138

28844

37515

41479

19336

44595

11400

18111

17384

114557

26960

26362

311517 -

HEIGHT
$=======$
5124
729
3245
2646
913
456
4585
660
465
353
727
1152
480
665
839
77
458
186
1318
248
161
7342

HT / AREA

$======$

0.024

0.028

0.038

0.026

0.039

0.038

0.034

0.037

0.024

0.023

0.025

0.031

0.012

0.034

0.019

0.007

0.025

0.011

0.012

0.009

0.006

0.024
\% AREA

$=====$

0.790

2.60

3.12

0.711

0.369

4.07

0.542

0.580

0.459

0.875

1.14

1.26

0.587

1.35

0.346

0.550

0.528

3.48

0.818

0.800

9.45
Compounds

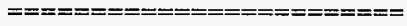

Decachlorobiphenyl

Total area $=3294957$

$$
\begin{aligned}
& =581,085 \\
& \hline 2,402,35
\end{aligned}
$$


Data File:/chem/gc2.i/052198.b/038f0401.d

Report Date: 29-May-1998 11:33

$$
\text { 222-S Laboratory }
$$

RECOVERY REPORT

Client Name:

Sample Matrix: SOLID

client SDG: 052198

Lab Smp Id: S98S000102MSD

Fraction: PEST

Level: LOW

Client Smp ID: $988 \mathrm{Tk} 103 \mathrm{MSD} / 200$

Data Type: GC MULTI COMP

Spikelist File: Aroclor-1254.spk

Method File: /chem/gc2.i/052198.b/rorapcb.m

Operator: Gerald Ross

SampleType: MSD

Misc Info: $988 T k 103 \mathrm{MSD} / 200$

\begin{tabular}{|c|c|c|c|c|}
\hline SPIKE COMPOUND & $\begin{array}{l}\text { CONC } \\
\text { ADDED } \\
\text { ug/Kg }\end{array}$ & $\begin{array}{c}\text { CONC } \\
\text { RECOVERED } \\
\text { ug/Kg }\end{array}$ & $\begin{array}{c}\% \\
\text { RECOVERED }\end{array}$ & LIMITS \\
\hline \multirow[t]{2}{*}{27 Aroclor-1254 } & 46300 & 84720,54200 & 138.57 & $50-150$ \\
\hline & $84.72-47.66$ & $37.06 / 46.30 \times 1$ & $80.05 \%$ & $14 \times 25-2 \%-x$ \\
\hline SURROGATE COMPOUND & $\begin{array}{l}\text { CONC } \\
\text { ADDED } \\
\mathrm{ug} / \mathrm{Kg}\end{array}$ & $\begin{array}{c}\text { CONC } \\
\text { RECOVERED } \\
\text { ug/Kg }\end{array}$ & $\begin{array}{c}\% \\
\text { RECOVERED }\end{array}$ & LIMITS \\
\hline $\begin{array}{l}\$ 29 \text { Tetrachloro-m-xyle } \\
\$ 30 \text { Decachlorobiphenyl }\end{array}$ & $\begin{array}{l}14800 \\
14800\end{array}$ & $\begin{array}{l}13800 \\
14200\end{array}$ & $\begin{array}{l}93.11 \\
95.73\end{array}$ & $\begin{array}{l}50-150 \\
50-150\end{array}$ \\
\hline
\end{tabular}


Data File: /chem/gc2.i/052198.b/038f0401.d

Report Date: 29-May-1998 13:25

Page 1

\section{2-S Laboratory}

Data file:/chem/gc2.i/052198.b/038f0401.d

Lab Smp Id: S98S000102MSD

Inj Date : 23-MAY-1998 05:40

operator : Gerald Ross

Smp Info : $988 \mathrm{Tk} 103 \mathrm{MSD} / 200$

Misc Info : $988 \mathrm{Tk} 103 \mathrm{MSD} / 200$

comment :

Method : /chem/gc2.i/052198.b/rcrapcb.m

Meth Date : 29-May-1998 13:08 gar Quant Type: ESTD

Cal Date : 22-MAY-1998 20:13 Cal File: 028f0401.d

Als bottle: 1

Dil Factor: 200.000

Integrator: HP Genie

Target Version: 3.10

Client Smp ID: $988 \mathrm{Tk} 103 \mathrm{MSD} / 200$

Inst ID: $902 . \mathrm{i}$

QC Sample: MSD

Compound Sublist: Aroclors.sub Sample Matrix: SOIL

\begin{tabular}{|c|c|c|c|c|c|c|}
\hline & & & CONCENTR & ATIONS & & \\
\hline & & & $\mathrm{ON}-\mathrm{COL}$ & FINAL & & \\
\hline RT & EXP RT OLT RT & RESPONSE & ( $n g)$ & $(u g / K g)$ & TARGET RANGE & RATIO \\
\hline$==$ & 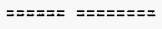 & $=== \pm====$ & =ニニニニニ= & $======+$ & $===\equiv=\overline{=}=\overline{=}==$ & $=====$ \\
\hline & clor -1016 & & & AS & $12674-11-2$ & \\
\hline
\end{tabular}

Peaks not detected for Quant. or Qual. signal(s).

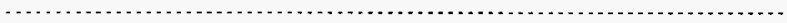

23 Aroclor -1221

CAS \#: $11104-28-2$

Peaks not detected for Quant. or Qual. signal(s).

24 Aroclor -1232

CAS \#: $1114-16-5$

Peaks not detected for Quant. or Qual. signal(s).

25 Aroclor -1242

CAS \# : $53469-21-9$

Peaks not detected for Quant. or Qual. signal(s).

\begin{tabular}{|c|c|c|c|c|c|c|c|}
\hline \multicolumn{4}{|c|}{26 Aroclor -1248} & \multicolumn{4}{|c|}{ CAS \#: $12672-29-6$} \\
\hline 18.688 & 18.675 & 0.013 & 93723 & 0.134 & 49700 & & 100.00 \\
\hline 19.686 & 19.679 & 0.007 & 43833 & 0.0675 & 25000 & $95.00-135.00$ & 46.77 \\
\hline 21.762 & 21.732 & 0.030 & 125280 & 0.147 & 54300 & $108.00-148.00$ & 133.67 \\
\hline \multicolumn{5}{|c|}{ Average of Peak Concentrations $=$} & \multicolumn{3}{|l|}{43000} \\
\hline \multicolumn{5}{|c|}{27 Aroclor- 1254} & \multicolumn{3}{|c|}{ CAS \#: $11097-69-1$} \\
\hline 21.762 & 21.767 & .0 .005 & 125280 & 0.154 & 56900 & & 100.00 \\
\hline 22.843 & 22.846 & -0.003 & 186525 & 0.173 & 64000 & $112.00-152.00$ & 148.89 \\
\hline 24.778 & 24.780 & -0.002 & 186728 & 0.193 & 71500 & $96.00-136.00$ & 149.05 \\
\hline \multicolumn{5}{|c|}{ Average of Peak CC } & \multicolumn{3}{|l|}{64200} \\
\hline
\end{tabular}


Data File: /chem/gc2.i/052198.b/038f0401.d Report Date: 29-May-1998 13:25

\begin{tabular}{|c|c|c|c|c|c|c|c|}
\hline \multirow[b]{3}{*}{ RT } & \multirow[b]{3}{*}{ EXP RT } & \multirow[b]{3}{*}{ DLT RT } & \multirow[b]{3}{*}{ RESPONSE } & \multicolumn{2}{|c|}{ CONCENTRATIONS } & \multirow[b]{3}{*}{ TARGET RANGE } & \multirow[b]{3}{*}{ RATIO } \\
\hline & & & & ON-COL & FINAL & & \\
\hline & & & & ng) & (ug/Kg) & & \\
\hline$==$ & $=====$ & $========$ & $==\Sigma=\Xi=\pi=$ & $==\Omega=ニ=\Omega$ & 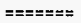 & 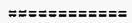 & $== \pm=$ \\
\hline
\end{tabular}

28 Aroclor -1260

CAS \#: $11096-82-5$

Peaks not detected for Quant. or Qual. signal(s).

-............................

CAS \#: $877-09-8$

$\begin{array}{lll}12.654 & 12.654 & 0.000\end{array}$

$\begin{array}{lll}549167 & 0.0372 \quad 13800\end{array}$

100.00

$\$ 30$ Decachlorobiphenyl

CAS \#: 2051-24-3

$\begin{array}{llll}42.723 & 42.728 & -0.005\end{array}$

$\begin{array}{lll}311517 & 0.0383 \quad 14200\end{array}$

100.00 
Data File: /chem/gc2.i/052198.b/038f0401.d

Report Date: 29-May-1998 13:25

Page 4

\section{2-S Laboratory}

TARGET COMPOUNDS

Client Name:

Lab Smp Id: S98S000102MSD

Sample Location:

Sample Date:

Sample Matrix: SOIL

Analysis Type: PEST

Data Type: GC MULTI COMP

Misc Info: $988 \mathrm{Tk} 103 \mathrm{MSD} / 200$
Client SDG: 052198

Client Smp ID: $988 \mathrm{Tk} 103 \mathrm{MSD} / 200$

Sample Point:

Date Received:

Quant Type: ESTD

Level: LOW

Operator: Gerald Ross

CAS NO.

COMPOUND

CONCENTRATION UNITS:

( $\mathrm{ug} / \mathrm{L}$ or $\mathrm{ug} / \mathrm{KG}$ ) $\mathrm{ug} / \mathrm{Kg}$

Q

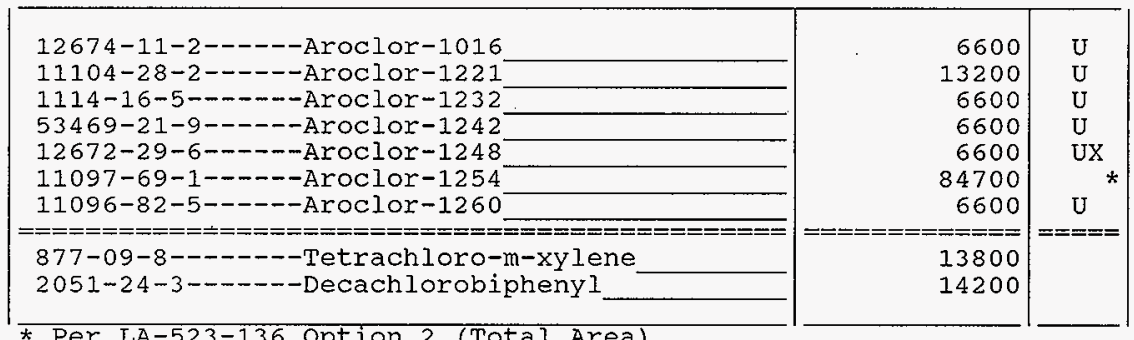


HNF-SD-WM-DP-306 REV. 0

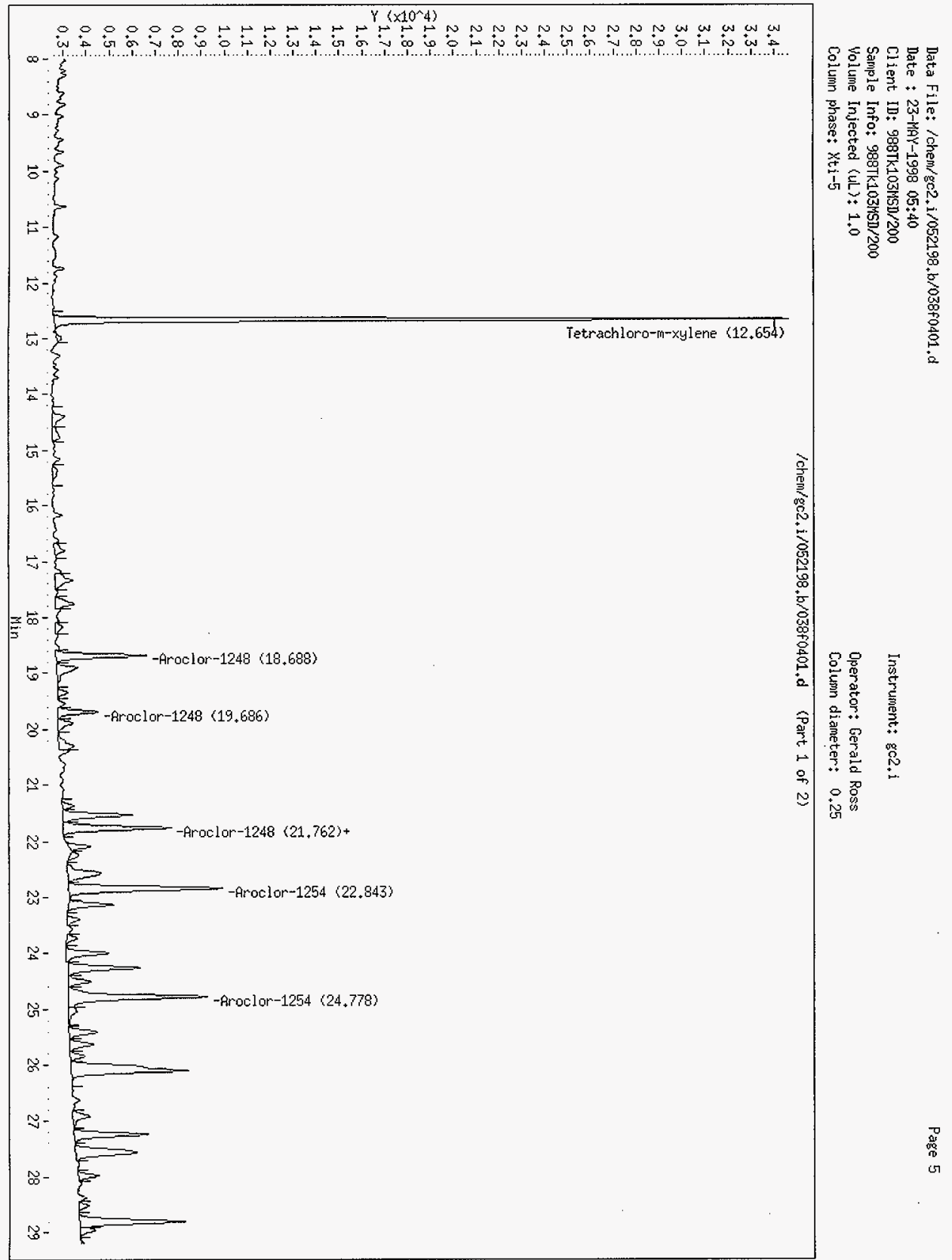


Data File: /chen/gc2, i/052198,b/o38f0401,d

Date : $23-M A Y-199805: 40$

Client ID: 988TK103MSD/200

Instrument; gc2, i

Sample Info: 988Tk103łSD/200

Volume Injected $(\mathrm{uL}) \div 1$. 0

Operator: Gerald Ross

Column phase: $x t i-5$

Colum diameter: 0.25

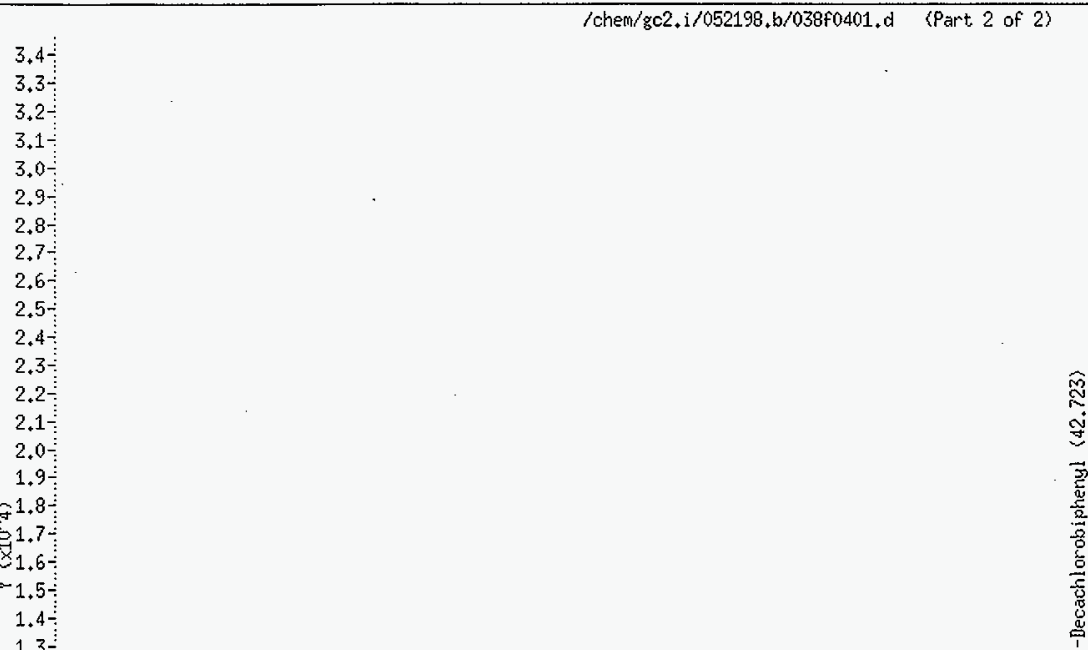

$1.3 \div$

1.2

1.1

1.0

0.9

0.8

0.7

0.5

0.4

0.3

0.2

0.1

$0.0-$

30

$31 \quad 3$

' 33

$34 \quad 35$

$\frac{1}{36}$

$37 \quad 3$

39

$40 \quad 41 \quad 42$

43

44

1
45

46

48 
HNF-SD-WM-DP-306, REV. 0

CONTINUING CALIBRATION VERIFICATION 
HNF-SD-WM-DP-306, REV. 0

2

THIS PAGE INTENTIONALLY LEFT BLANK 
Data File: /Chem/gc2.i/052198.b/040f0401.d

\section{2-S Laboratory}

Data file:/chem/gc2.i/052198.b/040f0401.d

Lab smp Id: $200 \mathrm{ng} / \mathrm{ml}$ A 1254

Inj Date : 23-MAY-1998 07:33

operator : Gerald Ross

Smp Info : $200 \mathrm{ng} / \mathrm{ml}$ A 1254

$\mathrm{Misc}$ Info: $200 \mathrm{ng} / \mathrm{ml} \mathrm{A} \mathrm{A} 254$

Comment :

Method : /chem/gc2.i/052198.b/rcrapcb.m

Meth Date : 29-May-1998 07:27 gar

Cal Date : 22-MAY-98 20:13

Als bottle: 1

DiI Factor: 1.000

Integrator: HP Genie

Target Version: 3.10

$== \pm=$

12.434

12.653

13.695

16.033

17.690

18.686

18.905

19.689

20.212

21.361

21.522

21.762

22.094

22.553

22.841

23.125

23.725

23.993

24.256

24.504

24.776

25.407

25.623

25.833

26.097

26.634

26.921

27.248

27.556

27.964

28.369

28.505

\section{AREA}

$==========$

10795

1438967

14152

918

14464

102051

23739

48942

123385

16941

75223

173822

25595

45065

224682

66507

10365

56221

103875

28928

207850

41618

30003

13726

227602

11897

21555

87978

114714

13238

14069

14170
HEIGHT

$====\approx==$

$==$

80555

737

205

4430

1018

2096

4924

668

2947

6508

973

1562

7892

2668

412

2214

3952

1135

7384

1391

1060

514

5388

403

681

3277

2951

609

520

482

Client. Smp ID: $200 \mathrm{ng} / \mathrm{ml}$ Al254

Inst ID: $g C 2, i$

Quant Type: AREA\%

Cal File: 028f0401.d

Compound Sublist: AR1254.sub Sample Matrix: WATER

HT/AREA \% AREA

$========$

0.046

0.056

0.052

0.000

0.014

0.043

0.043

0.043

0.040

0.039

0.039

0.037

0.038

0.035

0.035

0.040

0.040

0.039

0.038

0.039

0.036

0.033

0.035

0.037

0.024

0.034

0.032

0.037

0.026

0.046

0.037

0.034
Compounds

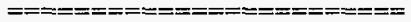

Tetrachloro-m-xylene

31.9

0.314

0.0204

0.321

2.26

0.526

1.08

2.74

0.376

1.67

3.85

0.568

0.999

4.98

1.47

0.230

1.25

2.30

0.641

4.61

0.923

0.665

0.304

5.05

0.264

0.478

1.95

2.54

0.294

0.312

0.314
Aroclor -1254

Aroclor -1254

Aroclor-1254 
Data File: /chem/gc2.i/052198.b/040f0401.d Report Date: 29-May-1998 09:32

Page 2

\begin{tabular}{|c|c|c|c|c|c|}
\hline$==\stackrel{\mathrm{RT}}{==}=$ & $\begin{array}{c}\mathrm{AREA} \\
=========\end{array}$ & $\begin{array}{c}\text { HEIGHT } \\
=========\end{array}$ & $\begin{array}{l}\mathrm{HT} / \mathrm{AREA} \\
======\end{array}$ & $\begin{array}{l}\% \text { AREA } \\
======\end{array}$ & $\begin{array}{l}\text { Compounds } \\
===================1\end{array}$ \\
\hline 28.798 & 143597 & 4981 & 0.035 & 3.18 & \\
\hline 28.946 & 18703 & 727 & 0.039 & 0.415 & \\
\hline 29.274 & 13155 & 414 & 0.031 & 0.292 & \\
\hline 30.352 & 41719 & 1275 & 0.031 & 0.925 & \\
\hline 30.993 & 13079 & 290 & 0.022 & 0.290 & \\
\hline 31.591 & 18754 & 651 & 0.035 & 0.416 & \\
\hline 32.460 & 9674 & 492 & 0.051 & 0.214 & \\
\hline 32.773 & 97 & 32 & 0.330 & 0.00215 & \\
\hline 34.139 & 25430 & 393 & 0.015 & 0.564 & \\
\hline 35.180 & 212 & 5 & 0.024 & 0.00470 & \\
\hline 37.813 & 6 & 0 & 0.000 & 0.000133 & \\
\hline 38.253 & 2842 & 98 & 0.034 & 0.0630 & \\
\hline 40.317 & 5278 & 160 & 0.030 & 0.117 & \\
\hline 41.033 & 58 & 13 & 0.224 & 0.00129 & \\
\hline 41.137 & 4312 & 4 & 0.001 & 0.0956 & \\
\hline 42.718 & 809941 & 17943 & 0.022 & 18.0 & Decachlorobiphenyl \\
\hline
\end{tabular}

Total area $=4509912$

$$
\begin{array}{r}
-1438967 \\
-809941 \\
\hline
\end{array}
$$

$2261,004 \quad 106.96 \%$ of $f: 10028 f 0401$ 
Data File: /chem/gc2.i/052198.b/040f0401.d

Report Date: 29-May-1998 12:00

\section{2-S Laboratory}

Data file: /chem/gc2.i/052198.b/040f0401.d

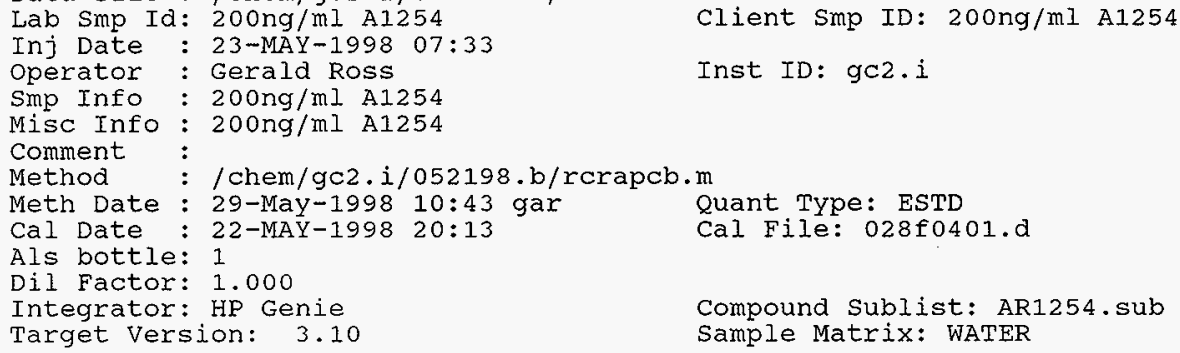

\begin{tabular}{|c|c|c|c|c|c|c|c|}
\hline & & & & CONCENTS & ATIONS & & \\
\hline & & & & $\mathrm{ON}-\mathrm{COL}$ & FINAL & & \\
\hline RT & EXP RT & DLT RT & RESPONSE & ( $n g)$ & ( $u g / L)$ & TARGET RANGE & RATIC \\
\hline$==$ & $======$ & $===x====$ & $=======[$ & $== \pm= \pm=0$ & $== \pm= \pm=\%$ & 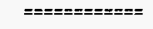 & $=x= \pm$ \\
\hline $27 \mathrm{Ar}$ & roclor-12 & & & & CAS \#: & $11097-69-1$ & \\
\hline 21.762 & 21.767 & -0.005 & 173822 & 0.213 & 4.26 & & 100.00 \\
\hline 22.841 & 22.846 & -0.005 & 224681 & 0.208 & 4.17 & $112.00-152.00$ & $129.2 \epsilon$ \\
\hline 24.776 & 24.780 & -0.004 & 207849 & 0.215 & 4.30 & $96.00-136.00$ & 119.58 \\
\hline & & Average of Peak & Concentr & ations $=$ & 4.24 & & \\
\hline$\$ 29 r$ & etrachlor & ro-m-xylene & & & CAS \# & $=877-09-8$ & \\
\hline 12.653 & 12.654 & -0.001 & 1438967 & 0.0976 & 1.95 & & 100.00 \\
\hline$\$ 300$ & ecachloro & obiphenyl & & & CAS \#: & $: 2051-24-3$ & \\
\hline 42.718 & 42.728 & $3-0.010$ & 809941 & 0.0996 & 1.99 & & 100.00 \\
\hline
\end{tabular}


Data File:/chem/gc2.i/052198.b/040f0401.d

Report Date: 29-May-1998 12:00

$$
\text { 222-S Laboratory }
$$

\section{RECOVERY REPORT}

Client Name:

Sample Matrix: LIQUID

Lab Smp Id: $200 \mathrm{ng} / \mathrm{ml}$ A1254

Level: LOW

Data Type: GC MULTI COMP

Spikelist File: RESOLUTION.spk

Method File:/chem/gc2.i/052198.b/rcrapcb.m

Client SDG: 052198

Fraction: PEST

client Smp ID: 200ng/ml A1254

operator: Gerald Ross

SampleType: SAMPLE

Quant Type: ESTD

Misc Info: $200 \mathrm{ng} / \mathrm{ml} \mathrm{A} 1254$

\begin{tabular}{|c|c|c|c|c|}
\hline SURROGATE COMPOUND & $\begin{array}{l}\text { CONC } \\
\text { ADDED }\end{array}$ & $\begin{array}{c}\text { CONC } \\
\text { RECOVERED }\end{array}$ & $\begin{array}{c}\% \\
\text { RECOVERED }\end{array}$ & LIMITS \\
\hline $\begin{array}{lll}\$ & 29 & \text { Tetrachloro-m-xyle } \\
\$ & 30 \text { Decachlorobiphenyl }\end{array}$ & $\begin{array}{l}2.00 \\
2.00\end{array}$ & $\begin{array}{l}1.95 \\
1.99\end{array}$ & $\begin{array}{l}97.59 \\
99.56\end{array}$ & $\begin{array}{l}50-150 \\
50-150\end{array}$ \\
\hline
\end{tabular}


Data File: /chem/gc2.i/052198.b/040f0401.d Report Date: 29-May-1998 12:00

\section{2-S Iaboratory}

TARGET COMPOUNDS

Client Name:

Lab Smp Id: $200 \mathrm{ng} / \mathrm{ml}$ A1254

Sample Location:

Sample Date:

Sample Matrix: WATER

Analysis Type: PEST

Data Type: GC MULTI COMP

Misc Info: $200 \mathrm{ng} / \mathrm{ml} \mathrm{A} 1254$
Client SDG: 052198

Client Smp ID: $200 \mathrm{ng} / \mathrm{ml}$ A1254

Sample Point:

Date Received:

Quant Type: ESTD

Leve 1: LOW

Operator: Gerald Ross

CAS NO.

COMPOUND

CONCENTRATION UNITS:

( $u g / L$ or $u g / K G$ ) ug/L

Q

$==========\left|\begin{array}{r}4.24 \\ 1.95 \\ 1.99\end{array}\right|===$




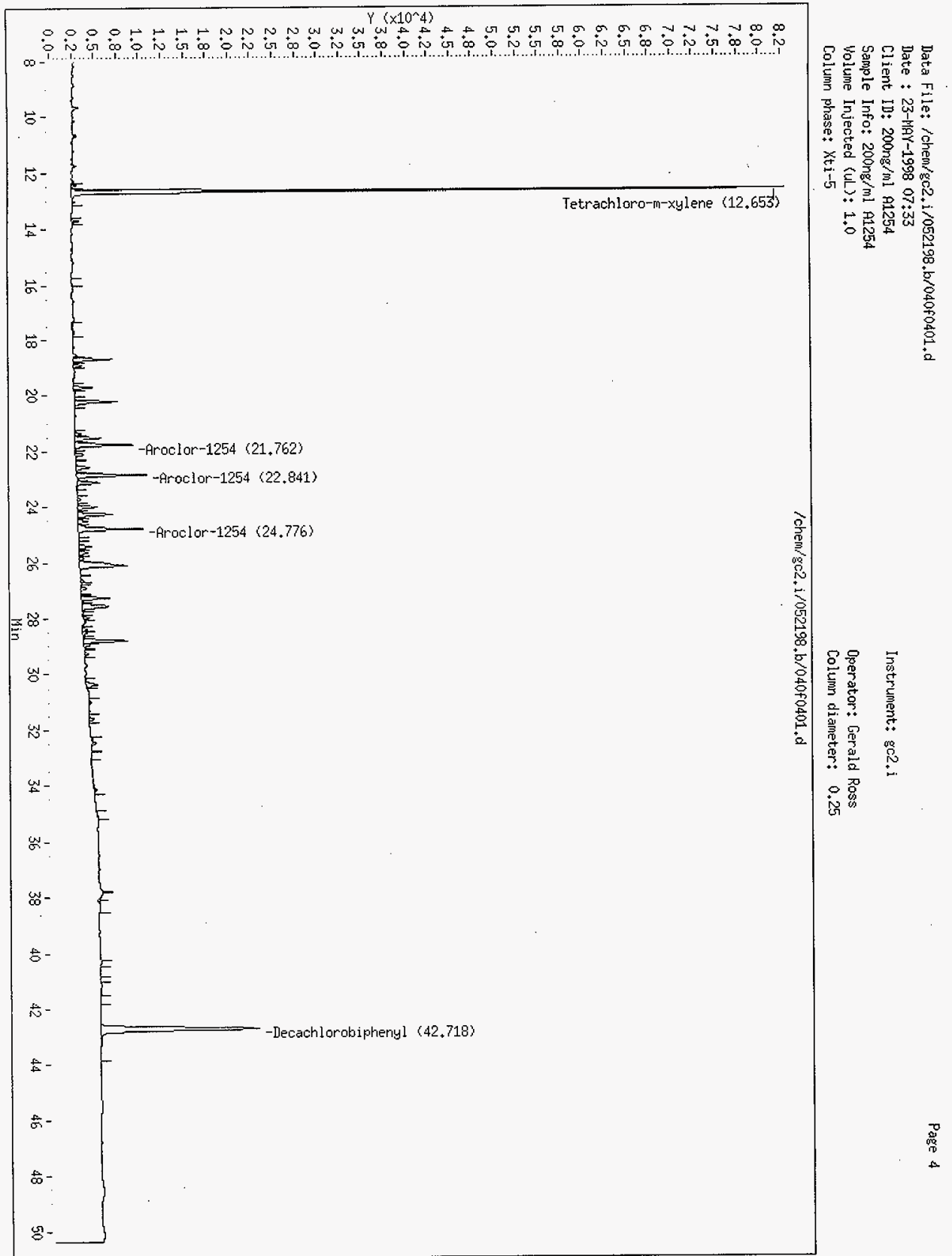


HNF-SD-WM-DP-306, REV. 0

SAMPLE BREAKDOWN 
HNF-SD-WH-DP-306, REV. 0

$=$

THIS PAGE INTENTIONALLY LEFT BLANK 


\section{S TK-103 Sample Breakdown}

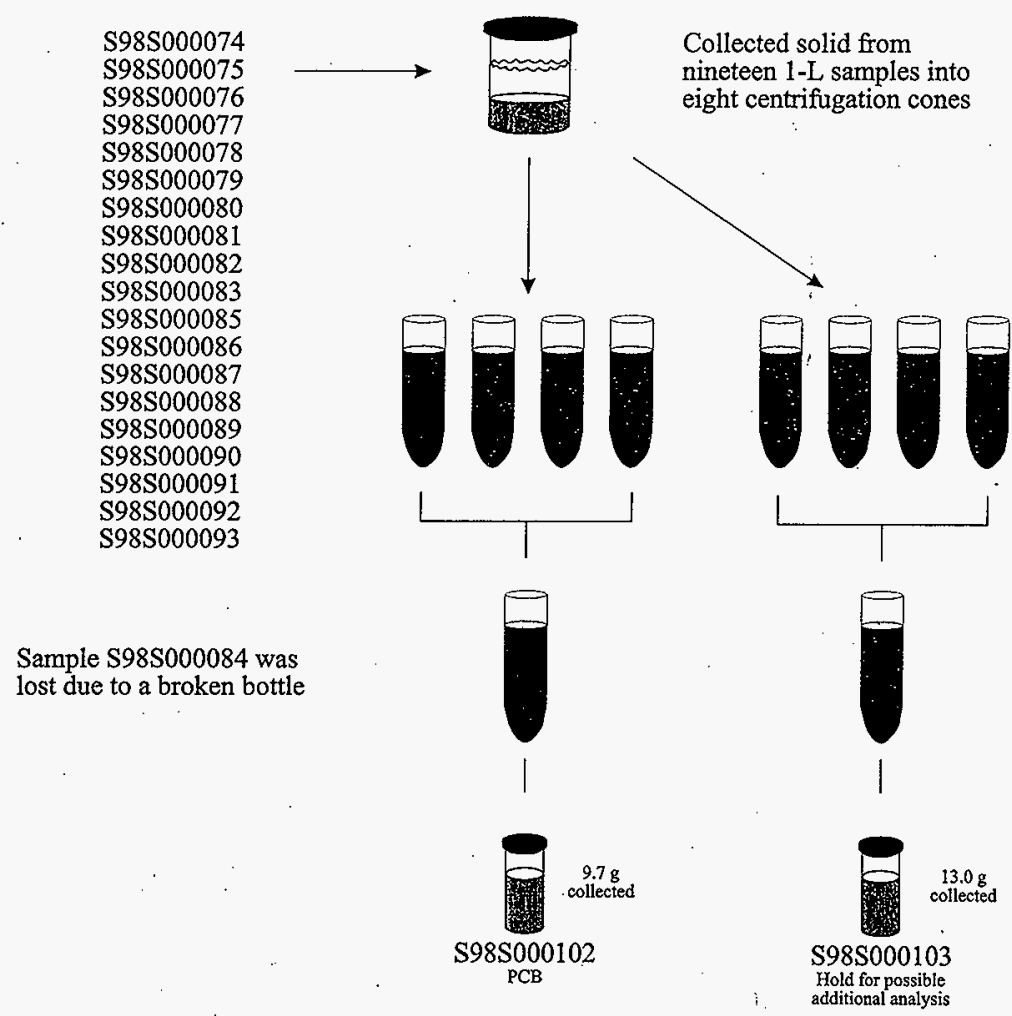


HNF-SD-WM-DP-306, REV. 0

REQUEST FOR SAMPLE ANALYSIS 
HNF-SD-WM-DP-306, REV. 0

$\bar{P}$

THIS PAGE INTENTIONALLY LEFT BLANK 


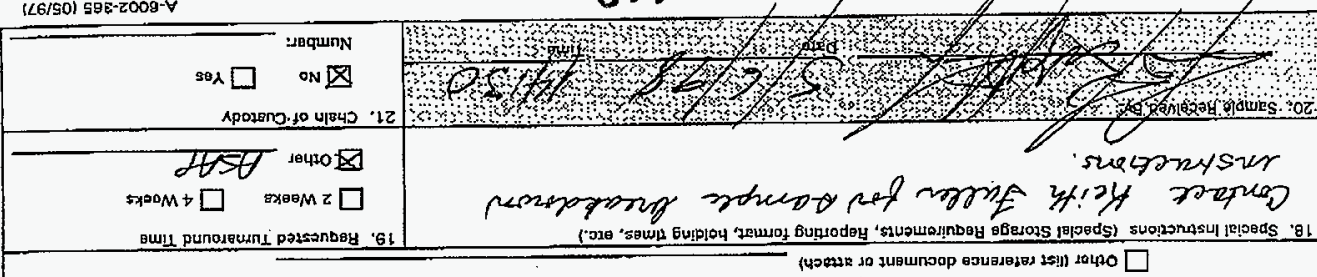

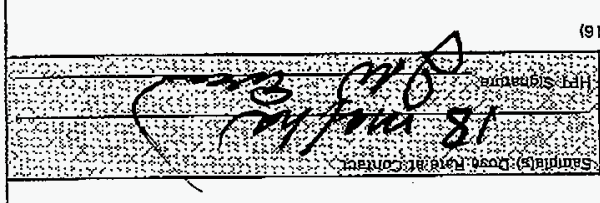

s, $9 J d$

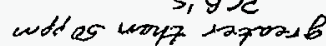

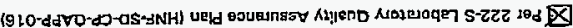

prumbay $00 \cdot \angle 1$

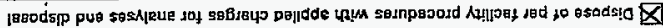

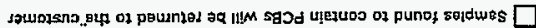
sew016n ox undoy $\square$ ag of pyosdxa top

uagigads!a 日duses 96 umousun $\bar{X}$

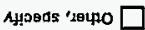
$\leftarrow$

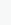

\section{(sBp00 28]) : :}

вA|10878 EẠฺOLOO

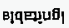

(рач)!w (pәupulazap Moy) :Z00Q (pघuluuesap MoL) :L009

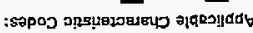

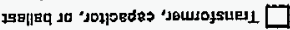

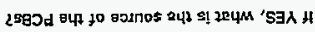
pelobogng ed8 sejd 听 $\square$

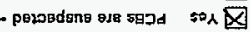
wad os Jeno $\quad \operatorname{sa} \square$ wdd $\operatorname{COS}$ denO

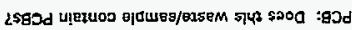

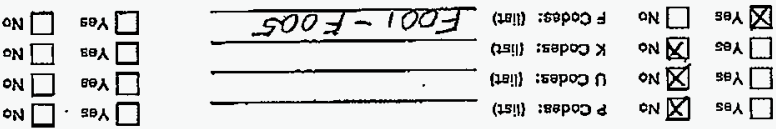

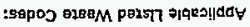

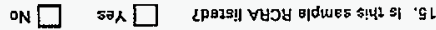

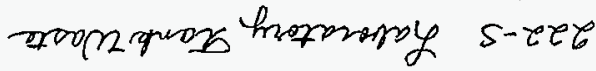

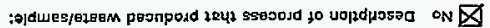
-ON SOSW PEUl!668 JHEH SOג $\square$

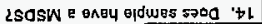

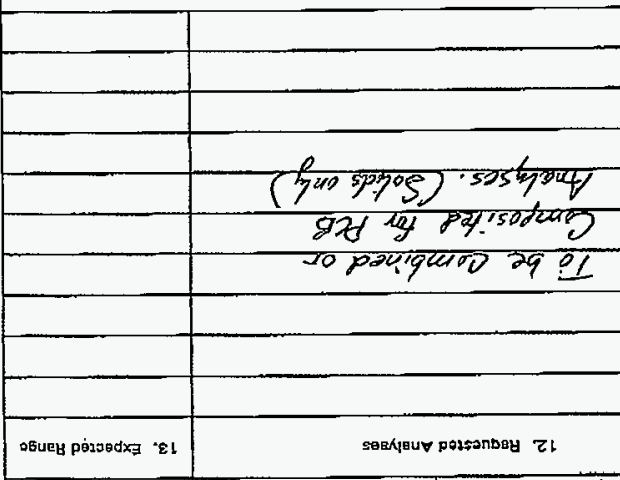

$$
\text { Jdasey }
$$

772 d Ag pөuluans 2

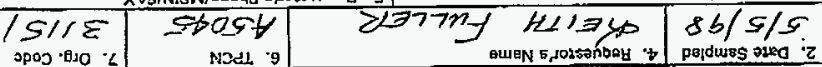

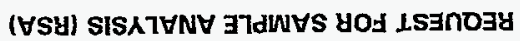

\begin{tabular}{|c|c|}
\hline \multicolumn{2}{|l|}{ 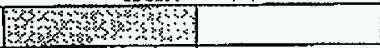 } \\
\hline 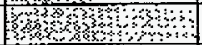 & \\
\hline molas & $8-86505-801$ \\
\hline 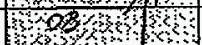 & $\angle-86505-801$ \\
\hline $6, b Z<\&$ \& & D) $-86505-401$ \\
\hline 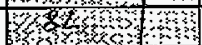 & $5-86505-801$ \\
\hline 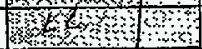 & $f-8 b 505-\varepsilon 01$ \\
\hline 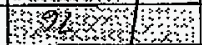 & $\varepsilon-86 s 0 s-\varepsilon 0 J$ \\
\hline & $z-86505-801$ \\
\hline 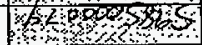 & $1-86505-801$ \\
\hline 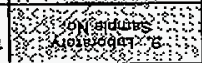 & 'ON II JPLHOLSחO '8 \\
\hline
\end{tabular}

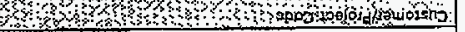
$56 / 2-501-X 1$ ụbiro 카wes 'b 


\section{REQUEST FOR SAMPLE ANALYSIS (RSA)}

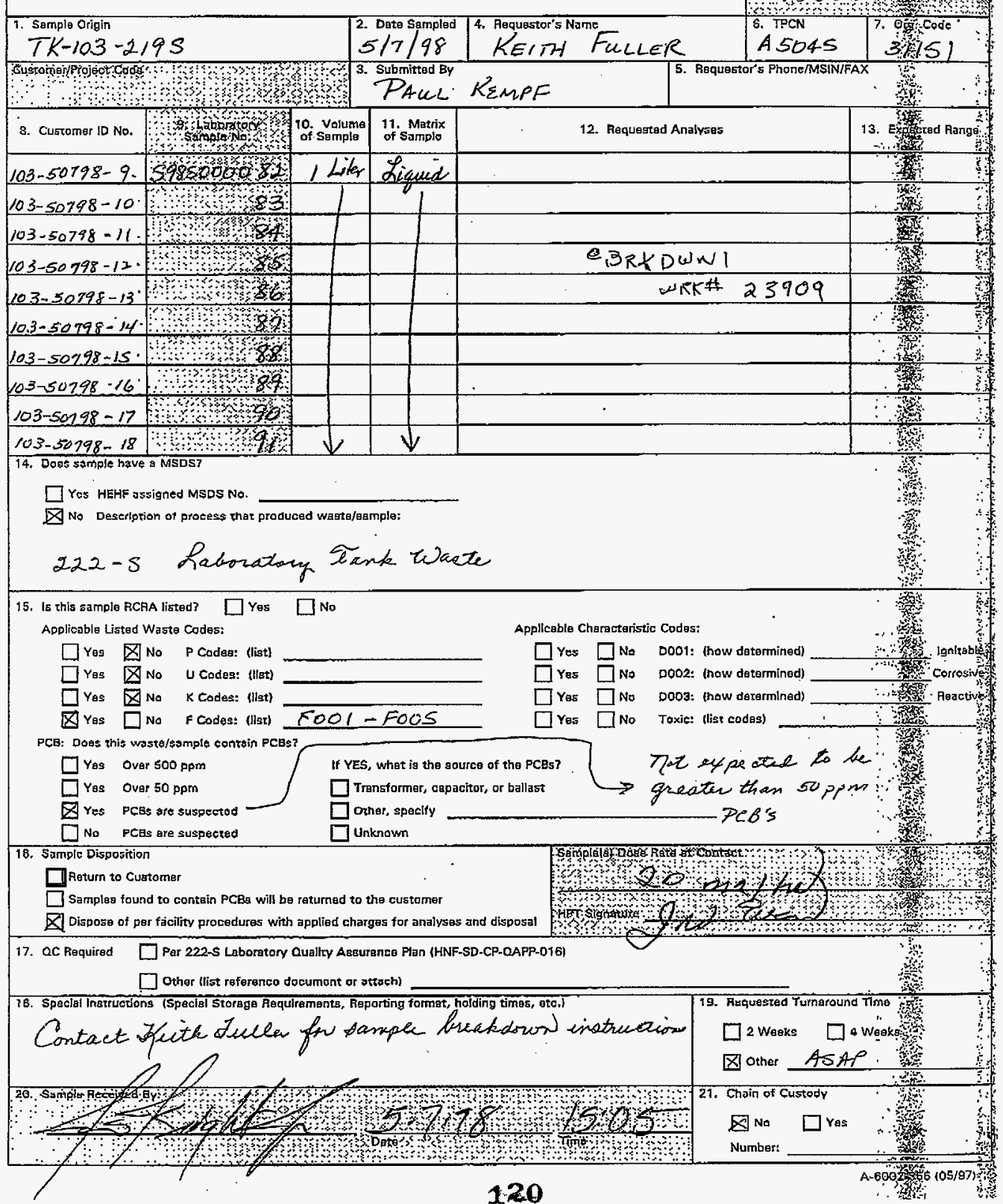




\section{REQUEST FOR SAMPLE ANALYSIS (RSA)}

\begin{tabular}{|c|c|c|c|c|c|c|c|}
\hline \multicolumn{4}{|c|}{$\begin{array}{l}\text { 7. Sample Oilgin } \\
T K-103-2,95\end{array}$} & \multicolumn{2}{|c|}{ 2. Data Sampled 4. Requestor's Nama } & $\begin{array}{l}\text { 8. TON } \\
A 5045\end{array}$ & $\begin{array}{c}7 . \text { Org. Cods } \\
3 / 151\end{array}$ \\
\hline $\begin{array}{l}\text { Customet } \\
\text { in }\end{array}$ & projencosts & कीका & $\mathrm{m}^{3}$ & Pacec & MMPF & 5. Requestor's Phone/MSII & \\
\hline 8. Cust & amer lo No. & 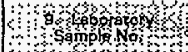 & \begin{tabular}{|c|c|} 
10. Voluma \\
of Sampla
\end{tabular} & $\begin{array}{l}\text { 11. Matux } \\
\text { of Samplas }\end{array}$ & 12. Request8 & Aralyaes & 13. Expestod Rango \\
\hline $103-50$ & $798-19$ & $3 \% S 0000$ & leiter & Lisuid & & & \\
\hline & 20 & 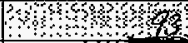 & & $D_{1}$ & & & \\
\hline$=$ & $-2 T$ & 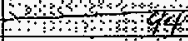 & - & & & & \\
\hline 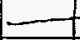 & 22 & hyophs & & & Doling to & $\checkmark P C \beta$ andused & \\
\hline & 23 & 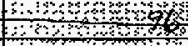 & $\Longrightarrow$ & & Lepana & le leme & \\
\hline- & $x+4$ & 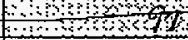 & - & & $\operatorname{arc} \alpha$ so & kis & \\
\hline & 25 & Pontar & - & & & . & \\
\hline & -26 & किकीये & & & & & \\
\hline$=$ & कन & dot & & & & & \\
\hline$\varepsilon$ & 28 & 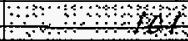 & 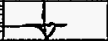 & $\downarrow$ & & & \\
\hline
\end{tabular}

$\square$ Yes HEHF assigned MSDS No.

W No Description of process that produced waste/sample:

222-S Labaratary Tank Whate

15. Is thls sample RCAA listed? $\square$ Yes $\square$ No

Appliceble Lister Waste Codes:

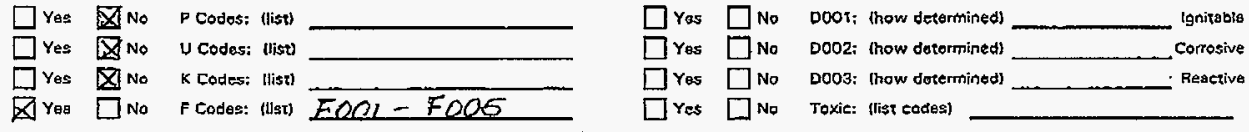

PCa: Does thls waste/sample contain PCBe?

$\begin{array}{ll}\square \text { Yes } & \begin{array}{l}\text { Over } 600 \mathrm{ppm} \\ \text { Yes }\end{array} \\ \square \text { Yeser } 60 \mathrm{ppm} \\ \square \text { No }\end{array}$

16. Sample Diaposition

$\square$ Rertith to Customer

प Semples tound to cantoin PCBs will bo raturnod to the customer

Q Dlspose of per tacllizy procodures with spplied charges for analysgs and disposal

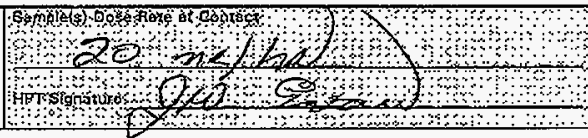

17. QC Required $\square$ Per 222-S Laboratory Quality Assurance Plan (HNF-SD-CP-QAPP-016)

$\square$ Other (llst reterance documen; or atzech)

18. Spetial lsstructions (Special Storage Requirsments, Feporting formot, holding times, etc.)

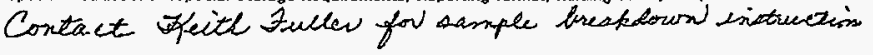
19. Fequested Tumaround Tima
$\square 2$ Weaks $\square 4$ Weaks
网

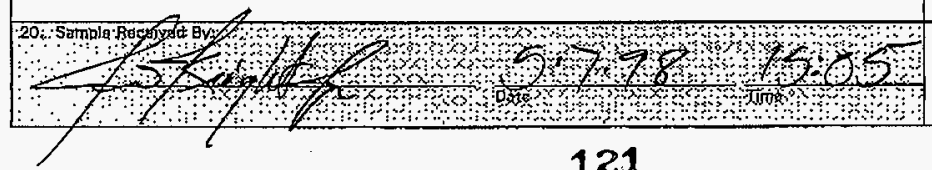

21. Chain of Custody

¿No $\square$ Yes

Nurnber: 


\section{DISTRIBUTION SHEET}

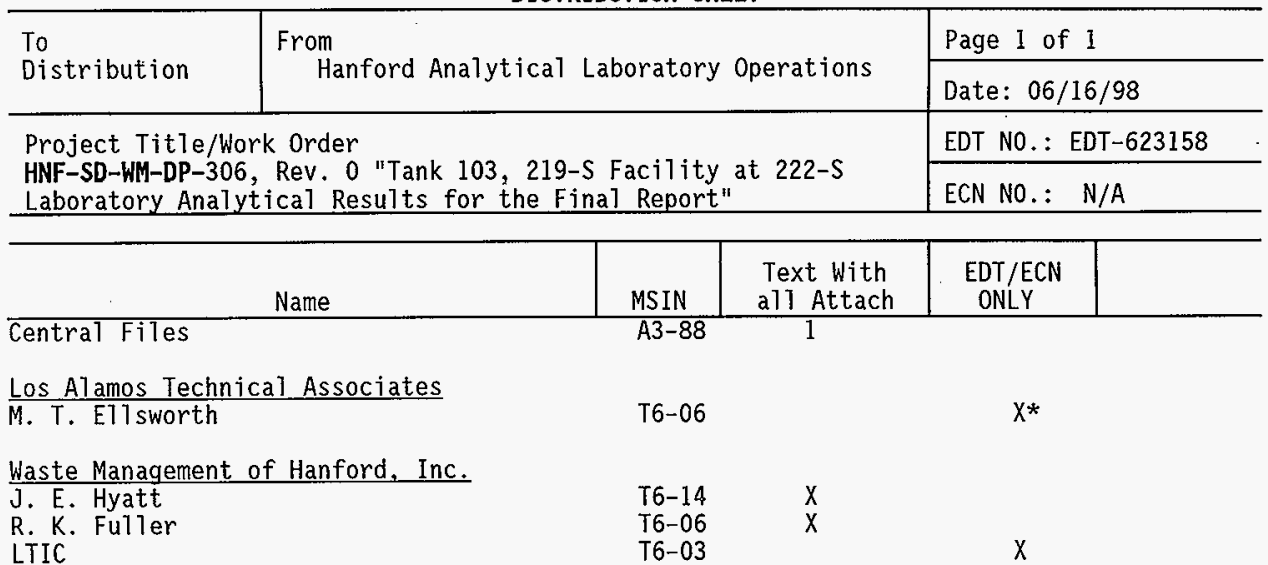

* Needs only releasing paperwork, not a copy of the released document. 